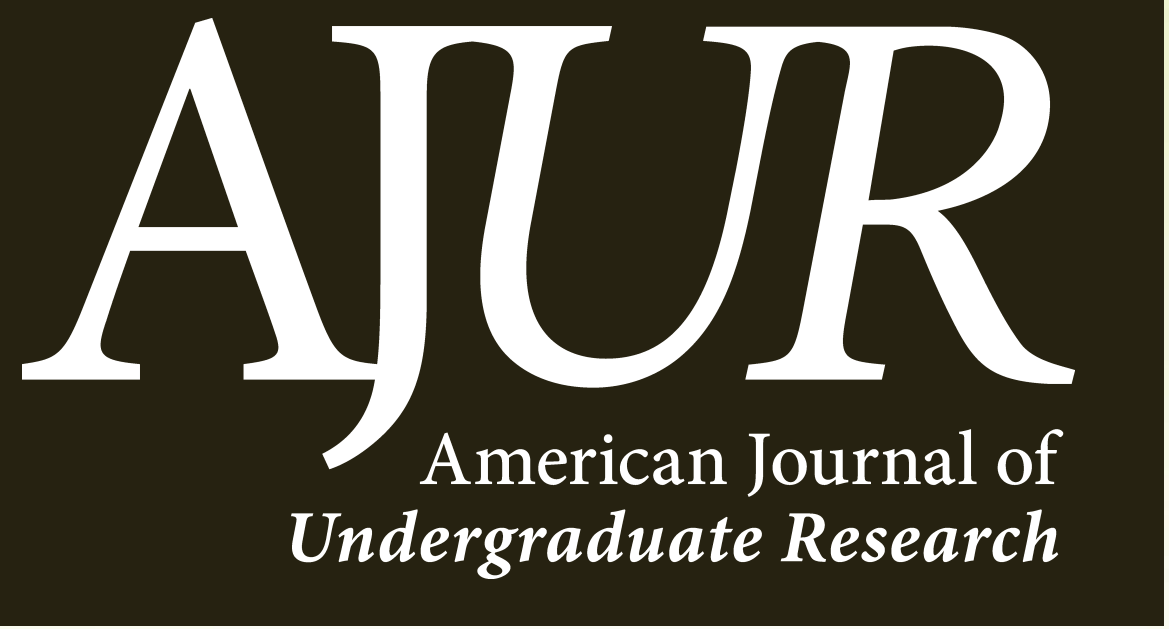

Volume 15 | Issue 1 | June 2018

www.ajuronline.org 


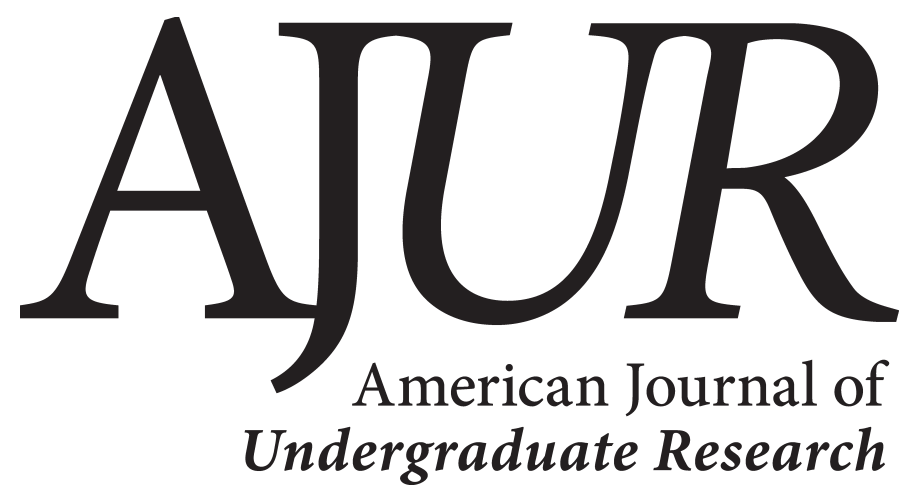

Volume 15 | Issue 1 | June 2018

www.ajuronline.org

AJUR History and Editorial Board

Special Thanks to AJUR's Sponsors

An All-Atomistic Molecular Dynamics Study to Determine the Structural Importance of Disulfide Bonds in Immunoglobulin G and Bovine Serum Albumin

Akshay Mathavan, Akash Mathavan, Michael E. Fortunato, \& Coray M. Colina

Syndecan Affects Odor Response as well as Learning and Memory in Drosophila melanogaster

Dena Arizanovska, Jonathan King, \& Karl G. Johnson

Investigation of Flow Rate on Chemical Bath Deposition of Silver

Films Inside Hollow Polymer Cylinders

M. Ehrsam, H. Ta₹, A. Malasi, C. Carr, \& R. Kalyanaraman

Feasibility of Man-Portable Power Generation Systems

Earl C. Allen \& Nelson Fumo

The Role of Gender Stereotypes in Gubernatorial Campaign Coverage

Karen Bjerre 
American Journal of Undergraduate Research (AJUR) is a national, peer-reviewed, open-source, quarterly, multidisciplinary student research journal. It is indexed internationally by EBSCO, and is listed via the Library of Congress under ISSNs of 1536-4585 (for print) and 2375-8732 (for web). The journal was established in 2002.

EDITORIAL TEAM Volume 15 / Issue 1 / June 2018

Dr. Kestutis G. Bendinskas, Editor, editor@ajuronline.org

Dr. Anthony Contento, Copy Editor

Rose Throop, Print

Daniel Laird, Web Master

EDITORIAL BOARD by subject area

ACCOUNTING

Dr. Dean Crawford, dean.crawford@oswego.edu

\section{ART HISTORY}

Dr. Lisa Seppi,

lisa.seppi@oswego.edu

ASTROPHYSICS

Dr. Shashi Kanbur,

shasbi.kanbur@oswego.edu

BEHAVIORAL NEUROSCIENCE

Dr. Aileen M. Bailey,

ambailey@smcm.edu

BIOCHEMISTRY

Dr. Pamela K. Kerrigan, pamela.kerrigan@mountsaintvincent.edu

Dr. Nin Dingra,

ndingra@alaska.edu

BIOENGINEERING

Dr. Jorge I. Rodriguez,

jorger@clemson.edu

\section{BIOINFORMATICS}

Dr. Kevin Daimi

daimikj@udmercy.edu

Dr. John R. Jungck,

jungck@udel.edu

Dr. Isabelle Bichindaritz,

ibicbind@oswego.edu

BIOLOGY, PHYSIOLOGY

Dr. David Dunn,

david.dunn@oswego.edu

\section{BIOLOGY, DEVELOPMENTAL}

Dr. Poongodi Geetha-Loganathan,

p.geethaloganathan@oswego.edu

\section{BIOLOGY, MICROBIOLOGY}

Dr. Peter Newell,

peter.newell@oswego.edu

\section{BOTANY}

Dr. William R. Bromer,

wbromer@stfrancis.edu

Dr. Julien Bachelier,

julien.bachelier@fu-berlin.de
CHEMISTRY

Dr. Alfredo Castro, castroa@felician.edu

Dr. Charles Kriley, cekriley@gcc.edu

Dr. Douglas Mulford, douglas.mulford@emory.edu

Dr. Vadoud Niri,

vadoud.niri@oswego.edu

COMMUNICATION DISORDERS AND SCIENCES

Dr. Kim Tillery,

Kim.Tillery@fredonia.edu

COMMUNICATION STUDIES

Dr. Jennifer Gerometta, jgerometta@iona.edu

\section{COMPUTER SCIENCES}

Dr. Dele Oluwade, deleoluwade@yahoo.com

Dr. Kevin Daimi,

daimikj@udmercy.edu

Dr. Levent Ertaul,

levent.ertaul@csueastbay.edu

Dr. Mais W Nijim,

Mais.Nijim@tamuk.edu

COMPUTATIONAL CHEMISTRY

Dr. Alexander Soudackov

alexander.soudackov@yale.edu

\section{ECOLOGY}

Dr. William R. Bromer, wbromer@stfrancis.edu

ECONOMICS

Dr. Elizabeth Schmitt,

elizabeth.schmitt@oswego.edu

EDUCATION

Dr. Marcia Burrell,

marcia.burrell@oswego.edu

EDUCATION, PHYSICS

Dr. Andrew D. Gavrin, agavrin@iupui.edu

ENGINEERING, ELECTRICAL

Dr. Michael Omidiora,

micbael.omidiora@nyu.edu

\author{
ENGINEERING, ENVIRONMEN- \\ TAL \\ Dr. Eileen M. Cashman, \\ eileen.cashman@bumboldt.edu
}

ENGINEERING, MANUFACTURING AND CONSTRUCTION, ROBOTICS

$$
\text { Dr. Haoyu Wang, }
$$
wanghao@mail.ccsu.edu

ENGINEERING, SOFTWARE Dr. Kevin Daimi, daimikj@udmercy.edu

ENVIRONMENTAL SCIENCES

Dr. Eileen M. Cashman, eileen.cashman@humboldt.edu

FILM AND MEDIA STUDIES

Dr. Lauren Steimer,
lsteimer@mailbox.sc.edu

HISTORY

Dr. Richard Weyhing, richard.weybing@oswego.edu

Dr. Murat Yasar,

muratyasar@oswego.edu

HONORARY EDITORIAL BOARD MEMBER

Dr. Lorrie Clemo, lorrie.a.clemo@gmail.com

JURISPRUDENCE

Bill Wickard, Esq,

William.Wickard@KLGates.com

KINESIOLOGY / EXERCISE SCIENCE

Dr. David Senchina,

david.sencbina@drake.edu

LITERARY STUDIES

Dr. Douglas Guerra,

douglas.guerra@oswego.edu

MATHEMATICS

Dr. John Emert,

emert@bsu.edu

Dr. Jeffrey J. Boats,

boatsjj@udmercy.edu

Dr. J.D. Phillips,

jophilli@nmu.edu

Dr. Dele Oluwade, deleoluwade@yahoo.com

Dr. Christopher Baltus, christopher.baltus@oswego.edu

Dr. Mark Baker, mark.baker@oswego.edu MEDICAL SCIENCES

Joan Newell, MD joannewellmd@gmail.com

METEOROLOGY

Dr. Steven Skubis, steven.skubis@oswego.edu

MUSIC

Dr. Juliet Forshaw, juliet.forshaw@oswego.edu

NANOSCIENCE AND CHEMISTRY

Dr. Gary Baker,

bakergar@missouri.edu

NEUROSCIENCE

Dr. Pamela E. Scott-Johnson, pscottj@calstatela.edu

PHYSICS

Dr. Carolina Ilie, carolina.ilie@oswego.edu Dr. Mohammad Islam, mohammad.islam@oswego.edu

POLITICAL SCIENCE

Dr. Katia Levintova,

levintoe@ungb.edu

PSYCHOLOGY

Dr. Joseph DW Stephens, jdstephe@ncat.edu

Dr. Pamela E. Scott-Johnson, pscottj@calstatela.edu

SOCIAL SCIENCES

Dr. Rena Zito, rzito@elon.edu

\section{STATISTICS}

Dr. Mark Ecker, mark.ecker@uni.edu

TECHNOLOGY, ENGINEERING

Dr. Recayi Pecen, regpecen@na.edu 


\section{SPECIAL THANKS}

AJUR is made possible through the volunteer efforts of our editorial team and assistance of our sponsors.

Support for this issue has been provided by the Office of the Provost at the State University of New York at Oswego. Thank you!

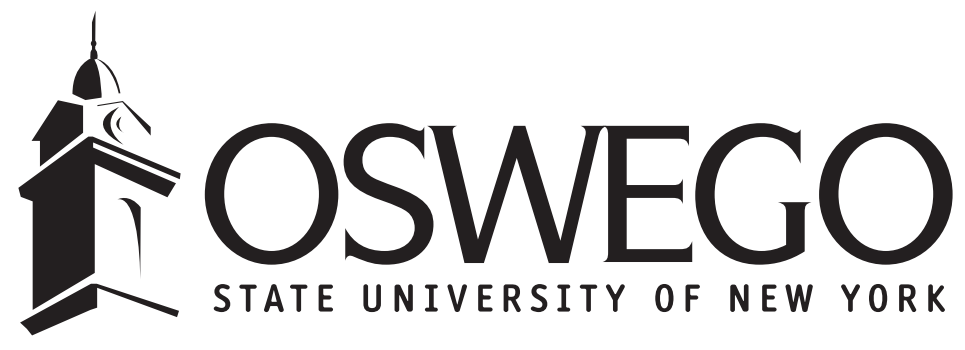

Please support undergraduate research

Request sponsorship information fromeditor@ajuronline.org 


\title{
An All-Atomistic Molecular Dynamics Study to Determine the Structural Importance of Disulfide Bonds in Immunoglobulin G and Bovine Serum Albumin
}

\author{
Akshay Mathavan ${ }^{a}$, Akash Mathavan ${ }^{a}$, Michael E. Fortunato ${ }^{b}$ \& Coray M. Colina*bc \\ ${ }^{a}$ Department of Biomedical Engineering, University of Florida, Gainesville, FL \\ ${ }^{b}$ Department of Chemistry, University of Florida, Gainesville, FL \\ 'Departments of Materials Science and Nuclear Engineering, University of Florida, Gainesville, FL \\ Students: amathavan1996@ufl.edu,amathavan2496@ufl.edu,mef231@chem.ufl.edu \\ Mentor:colina@chem.ufl.edu*
}

\begin{abstract}
A fully-atomistic molecular dynamics study was performed to determine the importance of disulfide bonds on the stability of immunoglobulin $\mathrm{G}(\mathrm{IgG})$ and bovine serum albumin (BSA). The transferability of a previous prescreening methodology to assess contributions from individual disulfide bonds on conformational stability was tested on both proteins. In IgG, it was apparent that inter-chain and intra-chain disulfide bonds play different roles in maintaining structure, evidenced by clear separation of interchain cysteine residues upon cleavage of disulfide bonds. In BSA, a set of double disulfide bonds required both to be broken in order to observe significant structural changes, equivalently seen in a previous study of human serum albumin (HSA), a structurally similar protein. Structural analysis of $\mathrm{IgG}$ showed deviations in distances between domains, while analysis of BSA suggested more local structural changes. This work helps confirm the efficacy and reproducibility of the prescreening methodology on both a novel, larger protein such as IgG and a more homologous (to HSA), globular protein such as BSA. The results provide insight into the role of specific disulfide bonds in the stability of IgG and BSA.
\end{abstract}

\section{KEYWORDS}

Molecular Dynamics; Atomistic Simulations; Immunoglobulin G; Bovine Serum Albumin; Disulfide Bonds

\section{INTRODUCTION}

Proteins are large molecules consisting of building-block units called amino acids. The linear sequence of amino acid residues for these biomolecules determines their three-dimensional structures. The complex assembly of multiple protein subunits can provide a vast array of functional capabilities ranging from enzyme catalysis to molecular transportation to antibody defense. Molecular flexibility introduced by regions of proteins lacking well defined structure can determine additional functional capacities; proteins with rigid components may serve as structural elements of a cell while those with flexible units may behave as hinges, springs, or levers. ${ }^{1}$

Disulfide bonds (SS bonds), covalent bonds between two cysteine residues, serve as important structural elements in proteins that ensure proper biological function. SS bonds establish conformational constraints on a peptide backbone, which can stabilize protein structure. ${ }^{2}$ Native SS bonds maintain properly folded conformations and destabilize improper or denatured formations through a decrease of conformational entropy. Bowman-Birk inhibitor proteins possess exposed hydrophobic motifs, yet seven SS bonds lock their native conformation and provide stability against common denaturants. ${ }^{3}$ Previous work has also emphasized the role of native SS bonds in determining the kinetics of folding pathways for proteins. ${ }^{4}$ The stabilizing effect of SS bonds can also be seen in the increased melting point of proteins.

Protein aggregation, the accumulation of misfolded proteins, has often been correlated with cell toxicity and a variety of human diseases. ${ }^{5-8}$ Protein aggregation in denaturing conditions has been suggested to result from the absence of native SS bonds. ${ }^{3,9,10}$ In many extracellular native proteins, including antibodies, receptors, and hormones, almost all sulfhydryl groups form SS bonds. ${ }^{3}$ SS bonds can form between residues within chains (intra-chain) or between chains (inter-chain) of proteins, and within domains (intra-domain) or between domains (inter-domain), with varied degrees of contribution towards structural stability. ${ }^{10-13}$ In 
denaturing environments, the presence of free cysteine residues in immunoglobulin G (IgG), an antibody, has been identified; the resulting lowered stability has been suggested to lead to non-native structures due to a high propensity for unfolding and aggregation. ${ }^{11}$ Reduction of SS bonds in lysozymes and bovine serum albumin (BSA) has yielded the formation of amorphous non-native structures, leading to protein aggregates. ${ }^{12}$

While previous experimental studies have identified the importance of SS bonds on the maintenance of protein tertiary structure, atomistic molecular dynamics (MD) simulations offer an alternative method to study the effects of both native and non-native SS bonds on protein structure and stability at the molecular level. ${ }^{13}, 14$ Previous MD simulations have studied the consequences of SS bond manipulation in signaling proteins, defense peptides, and biomarkers. ${ }^{15-18}$ Atomistic simulations presented in this study employ a prescreening methodology introduced in previous work by our lab which served to identify potentially significant SS bonds in human serum albumin (HSA) by initially looking at protein structures with all native SS bonds removed, thereby avoiding the computational cost of a systematic approach. ${ }^{19}$ Specifically, simulations of proteins with all native SS bonds removed were performed and separation distances between pairs of $\mathrm{S} \gamma$ atoms of those cleaved SS bonds were computed. Notable deviations in separation distances hinted towards those SS bonds as being critical to maintaining local structure, and thus guided the investigation of these specific bonds as being potentially responsible for structural changes.

This work extends the procedure to study two other proteins, IgG and BSA. Although structurally distinct, both proteins possess significant numbers of SS bonds, which have been experimentally studied and shown to contribute to protein aggregation upon reduction or cleavage. ${ }^{11-13}$ The large and flexible $\operatorname{IgG}$ protein presents a conformationally novel structure with which to apply the prescreening approach, and the significant structural homology between globular proteins BSA and HSA provides an opportunity to explore the relationship between protein structure and the role of SS bonds. Therefore, the purpose of this study was to apply the prescreening methodology, test its predictive capabilities, and understand the contributions of SS bonds at the atomistic level in two vastly different protein structures.

Immunoglobulins are large, Y-shaped glycoproteins. They are antibodies, major constituents of humoral immunity used to neutralize foreign toxins. $\operatorname{IgG}$ is the most abundant of five different immunoglobulins in humans $(\operatorname{IgA}, \operatorname{IgD}, \operatorname{IgE}, \operatorname{IgG}, \operatorname{IgM})$. $\operatorname{IgG}$ itself can be further subdivided into four classes ( $\mathrm{IgG} 1$, IgG2, IgG3, IgG4), of which IgG1 is the most prevalent serum antibody. ${ }^{20}$ The structure of IgG1 is defined by three globular domains formed by the folding of two light chains (L and M), each 215 residues in length, and two heavy chains ( $\mathrm{H}$ and $\mathrm{K}$ ), each 457 residues in length. Two of the three domains contain peptide sequences that bind to antigens $\left(\mathrm{F}_{\mathrm{ab}}\right.$ domains) and the other domain ( $\mathrm{F}_{\mathrm{c}}$ domain) can bind to other proteins. These domains are linked by the hinge region, which imparts a range of motion and flexibility. ${ }^{21}$ Although several structures of immunoglobulins have been published in the Protein Data Bank (PDB), only the 1HZH crystal structure captures the composition of human IgG1 antibodies. ${ }^{22,23}$ Due to the prevalence of IgG1 (hereafter simply referred to as $\operatorname{IgG}$ ) in human serum as well as its recent applications to monoclonal antibody therapies, the $1 \mathrm{HZH}$ crystal structure was selected as the model molecule for this study. ${ }^{24}$

For the purposes of this work, the hinge of $\operatorname{IgG}$ was studied as an independent region; therefore, IgG consisted of the three domains and one region: $\mathrm{F}_{\mathrm{ab}} 1$ (residues 1-230 of heavy chain H, 1-215 of light chain L), $\mathrm{F}_{\mathrm{ab}} 2$ (residues 1-230 of heavy chain $\mathrm{K}$, 1215 of light chain M), $\mathrm{F}_{\mathrm{c}}$ (residues 246-457 of heavy chain $\mathrm{H}$ and heavy chain $\mathrm{K}$ ), and the hinge region (residues 231-245 of heavy chain $\mathrm{H}$ and heavy chain $\mathrm{K}$ ). Residue numbers for IgG were defined with the relevant position and chain. IgG has 16 SS bonds, of which 12 are intra-chain and 4 are inter-chain. Two of the four inter-chain SS bonds are situated in the core hinge region, but experimental determination of the crystal structure for $1 \mathrm{HZH}$ showed that only one of the two pairs of cysteine residues were connected as SS bonds, indicating that the broken (unconnected) interchain SS bond may have been dynamic or a consequence of radiation damage. ${ }^{23}$ However, the broken SS bond likely has no functional significance as previous work has shown that only a single hinge SS bond is necessary for immunoglobulin proteins to be biologically active. ${ }^{25}$ Figure 1 presents the structure and distribution of SS bonds in $\mathrm{IgG}$, as well as the spatial arrangement of both the native connected and broken hinge SS bond. 

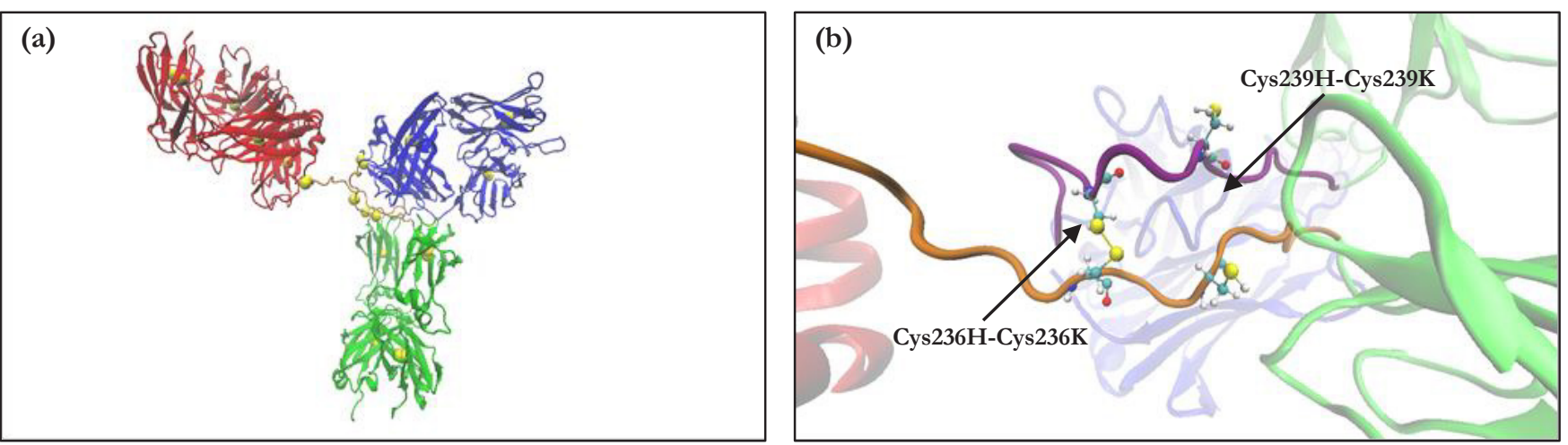

Figure 1. Cartoon representation of IgG (based on PDB entry 1HZH with homology modeling). ${ }^{23}$ Regions (a) are represented as: $\mathrm{F}_{\mathrm{ab}} 1$ (red), $\mathrm{F}_{\mathrm{ab}} 2$ (blue), $\mathrm{F}_{\mathrm{c}}$ (green), and hinge (orange). Sulfur atoms are depicted as yellow van der Waals (vdW) spheres. Chains of the hinge region (b) are represented as: residues 231H$245 \mathrm{H}$ (orange) and residues $231 \mathrm{~K}-245 \mathrm{~K}$ (purple). SS bonds are represented as ball-and-stick, with sulfur atoms as yellow. Connected (Cys236H-Cys236K) and broken (Cys239H-Cys239K) SS bonds are shown. The cartoons were created using Visual Molecular Dynamics. ${ }^{26}$

Serum albumins, the most prevalent circulatory proteins in mammals, function to preserve the body's osmotic pressure and are carrier proteins for compounds including hormones, fatty acids, and steroids. Serum albumins have been heavily studied due to their known primary and tertiary structures obtained through X-ray crystallography. They have also been the subject of significant drug delivery research, such as for applications in cancer drug therapy. ${ }^{27}$ Bovine Serum Albumin (BSA) is a heart shaped serum albumin isolated from cows. It has a predominantly $\alpha$ (alpha) helix structure with 583 residues and 17 intradomain SS bonds. ${ }^{28}$

BSA was modeled in this study using PDB entry $3 \mathrm{~V} 03 .{ }^{29} \mathrm{BSA}$ consists of three homologous domains, which are each divided into two subdomains: IA (residues 1-105), IB (residues 106-193), IIA (residues 194-295), IIB (residues 296-381), IIIA (residues $382-$ 494), and IIIB (residues 495-583). While specific subdomain residues were not listed for BSA, its close homology with HSA was used to match similar subdomain residues in BSA to HSA. Subdomain residue composition for HSA was obtained from PDB entry 1AO6. ${ }^{30}$ The SS bonds in BSA are well distributed throughout the protein, with at least two SS bonds per subdomain. All SS bonds in BSA are intra-domain (within a domain). Of the 17 SS bonds, 16 form double SS bonds, which are adjacent (next to each other) SS bonds in the primary amino acid sequence. Figure 2 presents the structure and SS bond distribution in BSA, as well as a double SS bond formed by Cys123-Cys168 and Cys167-Cys176.

(a)

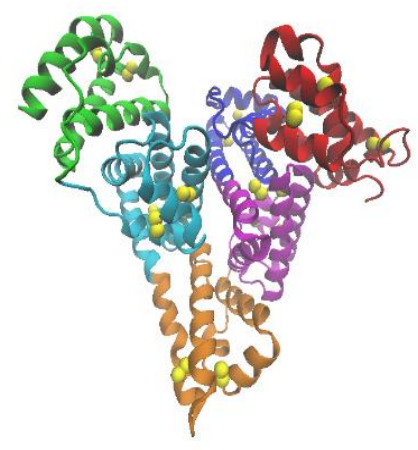

(b)

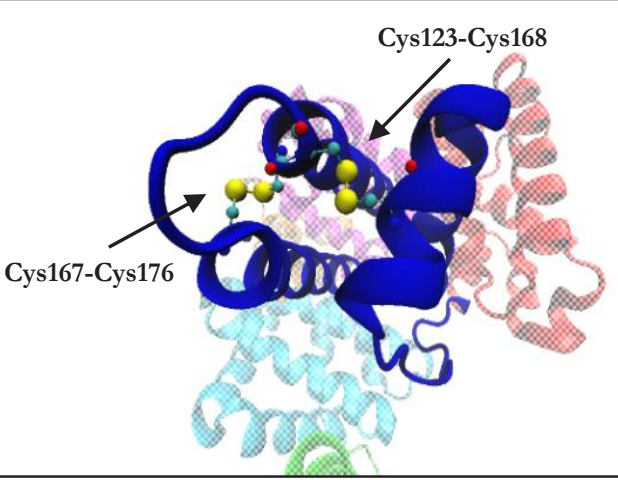

Figure 2. Cartoon representation of BSA (based on PDB entry 3V03). ${ }^{29}$ Regions (a) are represented as subdomains: IA (red), IB (blue), IIA (purple), IIB (orange), IIIA (cyan), and IIIB (green). Sulfur atoms are depicted as yellow van der Waals (vdW) spheres. Double SS bond (b) formed by Cys123-Cys168 and Cys167Cys176 is shown. SS bonds are represented as ball-and-stick, with sulfur atoms as yellow. The cartoons were created using Visual Molecular Dynamics. ${ }^{26}$

Due to the flexible nature of $\operatorname{IgG}$, connected by a hinge region with no stabilizing secondary structure, it was hypothesized that inter-chain SS bonds (such as that found in the hinge region) would be important to structural stability. There is a similarity of structure between BSA and HSA, with SS bonds conserved across the albumin proteins. Therefore, it was hypothesized that homologous SS bonds in BSA would be more important for maintaining protein structure. This study reveals conformational changes in IgG and BSA when native SS bonds are removed. The prescreening methodology is applied to guide cleavage of specific SS bonds, and resulting systems are analyzed. This work provides a predictive tool, with utility in investigating phenomena such as protein aggregation. 


\section{METHODS AND PROCEDURES}

Results for $1.6 \mu$ s of total simulation time using the AMBER16 simulation package are presented for IgG and BSA. ${ }^{31}$ The SANDER program was used for energy optimization and the GPU-accelerated PMEMD program was used for production runs. 31, 32 Initial structures were generated from entries in the Protein Data Bank (PDB entries 1HZH and 3V03 for IgG and BSA, respectively) using LEaP and the Amber 14SB force field.22, 23, 29, 33 Thirteen missing residues from heavy chain $\mathrm{K}$ in PDB crystal structure $1 \mathrm{HZH}$ were reconstructed using heavy chain $\mathrm{H}$ as a reference; the homology modeled $1 \mathrm{HZH}$ crystal structure is referred to as $1 \mathrm{HZH}^{+}$. For both proteins, the native disulfide (SS) bond connectivity was defined using information in the PDB entries. These disulfide bonds were those directly observed in experiment. ${ }^{23,}{ }^{29}$ Eight total simulations were performed with various SS bond connectivity for each protein. Table 1 summarizes the cleavage of SS bonds in each MD simulation performed in this study. Non-zero charges were neutralized for each protein by adding counterions; $22 \mathrm{Cl}^{-}$and $16 \mathrm{Na}^{+}$ions were needed to neutralize IgG and BSA, respectively. Each protein was solvated with explicit TIP3P water in a truncated octahedral box with a buffering distance of 10 Angstroms $(\AA)$ and $20 \AA$ for IgG and BSA, respectively, from the edge of the protein to any edge of the box. ${ }^{34,35}$

\begin{tabular}{|c|c|c|c|c|}
\hline Simulation Run & PDB & Protein & Length (ns) & Cleaved SS Bonds \\
\hline $\mathbf{1}$ & $1 \mathrm{HZH}^{+}$ & Immunoglobulin G & 200 & None \\
\hline $\mathbf{2}$ & $1 \mathrm{HZH}^{+}$ & Immunoglobulin G & 200 & All \\
\hline $\mathbf{3}$ & $1 \mathrm{HZH}^{+}$ & Immunoglobulin G & 200 & None \\
\hline $\mathbf{4}$ & $3 \mathrm{~V} 03$ & Bovine Serum Albumin & 200 & All \\
\hline $\mathbf{5}$ & $3 \mathrm{~V} 03$ & Bovine Serum Albumin & 200 & Cys123-Cys168 \\
\hline $\mathbf{6}$ & $3 \mathrm{~V} 03$ & Bovine Serum Albumin & 200 & Cys167-Cys176 \\
\hline $\mathbf{7}$ & $3 \mathrm{~V} 03$ & Bovine Serum Albumin & 200 & Cys123-Cys168 and \\
\hline $\mathbf{8}$ & $3 \mathrm{~V} 03$ & Bovine Serum Albumin & 200 & Cys \\
\hline
\end{tabular}

Table 1. Summary of Molecular Dynamics simulations, detailing crystal structures, simulation lengths, and SS bond state for each run. Simulations were performed for a total time of $1.6 \mu \mathrm{s}$.

Energy minimization was conducted in two steps: 1) protein atoms were restrained to initial coordinates with a harmonic potential of $500.0 \mathrm{kCal} / \mathrm{mol}$ while the protein-water interface relaxed and 2) the whole system was relaxed with no restraints. Minimization consisted of steepest descent succeeded by conjugate gradient, each for an equal number of steps (total steps: 20000 for $1 \mathrm{HZH}$ and 25000 for BSA), with a $12 \AA$ cutoff for all nonbonded interactions. The particle mesh Ewald algorithm was used to consider long range electrostatic interactions. ${ }^{36-38}$ Then, each system was heated continuously from 0 to $300 \mathrm{~K}$ for a period of 40 ps, while protein atoms were restrained with a weak harmonic potential of $10.0 \mathrm{kCal} / \mathrm{mol}$.

Heating of systems was followed by NPT (constant number of atoms, pressure, and temperature) simulations at $300 \mathrm{~K}$, employing the Langevin thermostat and a collision frequency of $2.0 \mathrm{ps}^{-1}$. MD simulations were performed with periodic boundary conditions. The pressure was maintained at 1 atmosphere with a pressure relaxation time of 2 ps. The SHAKE Algorithm was applied during all MD simulations to place constraints on any bond containing hydrogen atoms. This permitted a time step of $2 \mathrm{fs}$, and system information was recorded every 20 ps. The CPPTRAJ module of AmberTools was used for analysis of the trajectories, including root mean squared deviation (RMSD), atomic distance and vector analysis. ${ }^{31,39}$

RMSDs provided information about structural changes in the proteins. Atomic distances between sulfur atoms in pairs of cysteine residues were analyzed to identify the effects of SS bond cleavage on protein structure. Systems of each protein with all native SS bonds cleaved (simulation runs 2 and 5) were prepared and simulated. Separation distances between S $\gamma$ atoms were calculated and compared to equilibrium bond lengths. Following identification of $S \gamma$ atom pairs with noticeably large $(>6 \AA)$ separation distances, atomic simulations were performed with these specific SS bonds removed. Vector analysis and imaging in 
Visual Molecular Dynamics (VMD) were used to investigate structural deviations with respect to changes in distances between domains of proteins.

\section{RESULTS AND DISCUSSION}

\section{Root Mean Squared Deviation}

Root mean square deviations (RMSDs) for backbone atoms (C $\alpha, \mathrm{C}, \mathrm{N})$ in $\operatorname{Ig} \mathrm{G}$ and BSA were calculated with reference to the initial crystal structures (in the case of $\operatorname{IgG}, 1 \mathrm{HZH}^{+}$was referenced). RMSDs measure average distances between atoms of a protein and reference structure throughout a simulation trajectory, providing information about possible conformational changes. These calculations were conducted for IgG structures in simulation runs 1, 2 and 3 (with SS Bonds, without SS Bonds, and without Cys236H-Cys236K, respectively) described in Table 1.

Results of RMSD calculations are presented in Figure 3. In all simulations, an increase in RMSD values was observed during the first $\sim 20 \mathrm{~ns}$, which can be attributed to the change in environment from X-ray crystallographic conditions to a solvated ambient environment. After $\sim 30$ ns, RMSD values in simulation run 1 fluctuated around an average of $7 \AA$. RMSD values in simulation run 2 increased for $\sim 100 \mathrm{~ns}$, after which values fluctuated around an average of $8 \AA$. The RMSD values for simulation run 3 increased initially (from a change to solvated environment), and increased again after $\sim 60$ ns. After $\sim 120$ ns, RMSD values fluctuated around an average of $10 \AA$. Table 2 details RMSD information for simulation runs when no noticeable increase in RMSD values were observed (after $\sim 30, \sim 100$, and $\sim 120$ ns for simulation runs 1, 2, and 3, respectively).

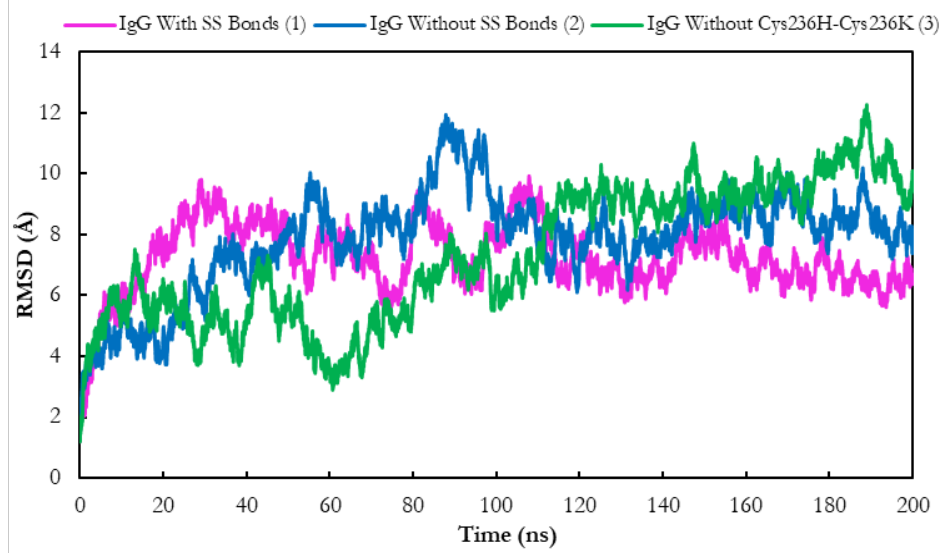

Figure 3. RMSDs for three cases of $\operatorname{IgG}$ simulations studied in this work, showing evolution over $200 \mathrm{~ns}$ for structures of $\operatorname{IgG}$ with SS Bonds (simulation run 1 , in pink), without SS Bonds (simulation run 2, in blue), and without Cys236H-Cys236K (simulation run 3, in green), referencing crystal structure $1 \mathrm{HZH}^{+}$.

\begin{tabular}{|c|c|c|}
\hline Simulation Run & Average $(\AA)$ & Standard Deviation $(\AA)$ \\
\hline $\mathbf{1}$ & 6.9 & 0.6 \\
\hline $\mathbf{2}$ & 8.4 & 0.6 \\
\hline $\mathbf{3}$ & 9.6 & 0.7 \\
\hline
\end{tabular}

Table 2. RMSDs for IgG simulations after no noticeable increases in values occurred. Provided are average and standard deviation of values for structures of IgG with SS Bonds (simulation run 1), without SS Bonds (simulation run 2), and without Cys236H-Cys236K (simulation run 3), referencing crystal structure $1 \mathrm{HZH}^{+}$.

For simulation runs 1 and 2, RMSDs were also calculated for each domain of IgG. Figure 4 presents the calculated RMSDs for each domain in simulation runs 1 and 2 . In $\mathrm{F}_{\mathrm{ab}} 1, \mathrm{~F}_{\mathrm{ab}} 2$, and $\mathrm{F}_{\mathrm{c}}, \mathrm{RMSD}$ values for both simulation runs initially increased and then fluctuated around an average of 1 to $3 \AA$. In the hinge region, RMSDs increased and fluctuated around $\sim 5$ and $\sim 4 \AA$ for simulation runs 1 and 2, respectively. Domain RMSD values were relatively smaller than those for the whole protein, agreeing with previous work that analyzed the protein structure of $\operatorname{IgG}{ }^{40}$ 

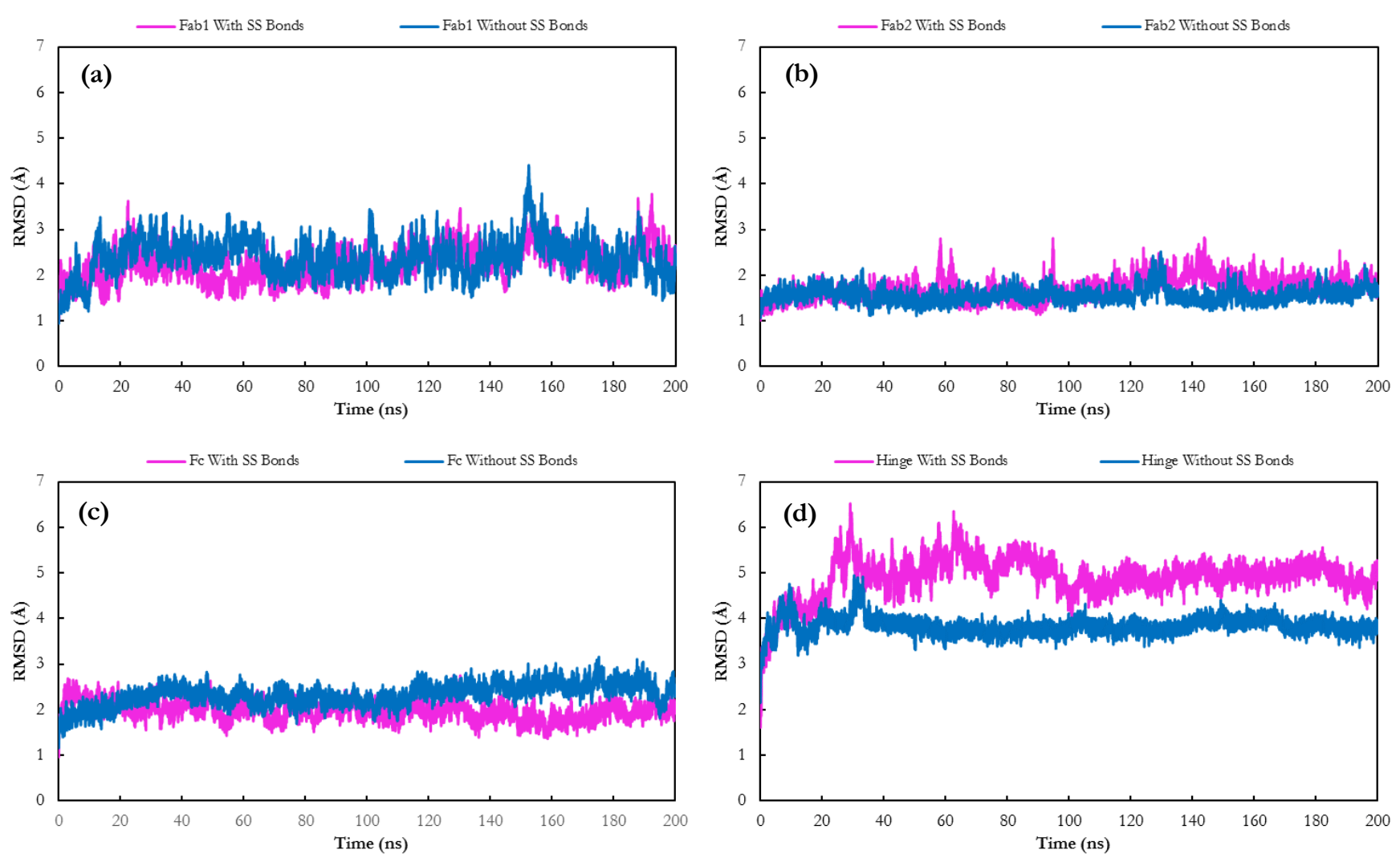

Figure 4. IgG domain RMSDs for backbone atoms during simulation runs 1 and 2 for: (a) $F_{a b} 1$ domain, (b) $F_{a b} 2$ domain, (c) $F_{c}$ domain, and (d) hinge region. Results are presented for IgG with SS Bonds (simulation run 1, in pink) and without SS Bonds (simulation run 2, in blue), referencing crystal structure $1 \mathrm{HZH}^{+}$.

The RMSD analyses for IgG structures, shown in Table 2, provided information about the relative stability of the protein and possible conformational changes that occurred over 200 ns. Average RMSD calculations shown in Figure 3 showed that values for the entire IgG backbone were noticeably higher (compared to domain RMSDs) than what would be expected for proteins in solvated conditions. Generally, larger collections of residues (as with the domains) would show larger backbone deviations. RMSD values for each domain of IgG, in Figure 4, were stable and lower than those of the whole protein, confirming the stability of the protein domains in solution and indicating that the relative motions of the domains contributed to the high average RMSD of the entire protein backbone structure. It was also evident that the hinge region, which contains a low number of residues, displayed higher backbone deviations in comparison to other domains. These results agree with well-studied segmental motion in IgG, in which the hinge region is known to impart a high degree of molecular flexibility. ${ }^{21}$ Although RMSD values for all three IgG simulations reached a similar range of 6 to $10 \AA$, an increased equilibration time for simulation runs with non-native SS bond connectivity was observed. These longer equilibration timescales indicate that structural changes likely occurred.

RMSD calculations were also performed for BSA structures in simulation runs 4, 5, 6, 7, and 8, described in Table 1. Results for all simulation runs are shown in Figure 5. In all cases, an increase in RMSD values was seen during the first $\sim 10$ ns due to the change to a solvated environment. After $\sim 10$ ns, no noticeable deviation in RMSD was observed for all BSA simulation runs; with values fluctuating between 2 to $4 \AA$. Table 3 presents average RMSD values for simulation runs $4-8$ after $\sim 10$ ns.

Figure 5 shows that BSA RMSD values for all simulation runs (4-8) increased for only 10 ns before equilibrating for the remainder of simulation time. When compared to $\operatorname{IgG}$, the RMSDs showed relatively quick equilibration times and lower average values. This can be attributed to the globular nature of BSA, imposing a relative inflexibility. The results from the RMSD analysis did not indicate large scale structural changes even when cleaving SS bonds in BSA; however, that did not rule out possible changes to local protein structure which could play a role in ligand binding. Analysis of HSA, a structurally homologous protein, from previous work by our lab showed comparable RMSD values, with a range of 2 to $4 \AA$ in corresponding simulations. ${ }^{19}$ 


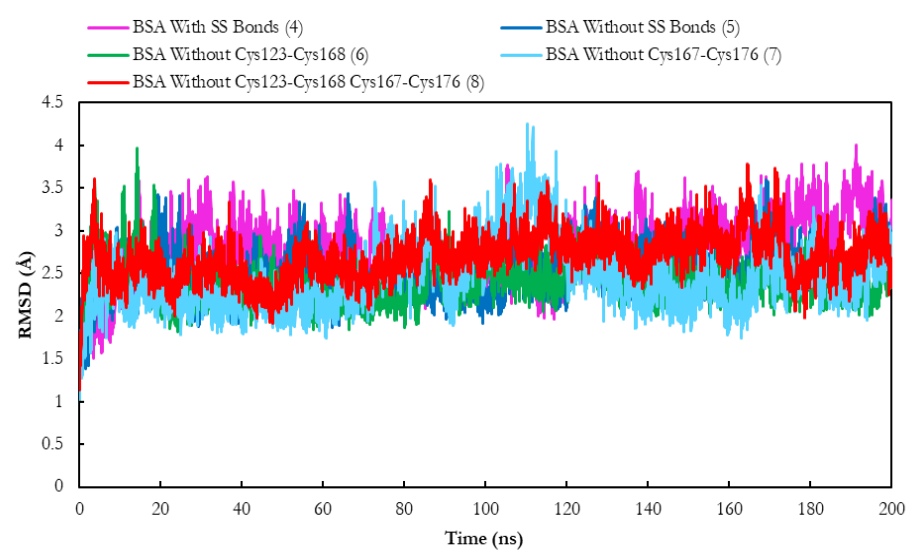

Figure 5. RMSDs for all runs of BSA simulations studied, showing evolution over $200 \mathrm{~ns}$ for structures of BSA with SS Bonds (simulation run 4, in pink), without SS Bonds (simulation run 5, in blue), without Cys123-Cys168 (simulation run 6, in green), without Cys167-Cys176 (simulation run 7, in teal), and without Cys123-Cys168 and Cys167-Cys176 (simulation run 8, in red), referencing crystal structure 3V03.

\begin{tabular}{|c|c|c|}
\hline Simulation Run & Average $(\AA)$ & Standard Deviation $(\AA)$ \\
\hline $\mathbf{4}$ & 2.8 & 0.4 \\
\hline $\mathbf{5}$ & 2.5 & 0.3 \\
\hline $\mathbf{6}$ & 2.3 & 0.2 \\
\hline $\mathbf{7}$ & 2.4 & 0.4 \\
\hline $\mathbf{8}$ & 2.6 & 0.3 \\
\hline
\end{tabular}

Table 3. RMSD values for simulations of BSA after $10 \mathrm{~ns}$. Provided are average and standard deviation of values for structures of BSA with SS Bonds (simulation run 4), without SS Bonds (simulation run 5), without Cys123-Cys168 (simulation run 6), without Cys167-Cys176 (simulation run 7), and without Cys123-Cys168 and Cys167-Cys176 (simulation run 8), referencing crystal structure 3V03.

\section{Disulfide Bonds}

Analyses of separation distances for simulation runs 1, 2, 4, and 5 in Table 1 (IgG and BSA with and without native SS bonds) provided information about SS bonds in both protein structures. SS bonds can be very important in maintaining protein structure; however, intermolecular interactions such as hydrogen bonding or the exclusion of water from hydrophobic regions can also help maintain protein structure. Analysis of experimental data have shown that in native, bonded states, sulfur atoms in pairs of cysteine residues maintain an average distance of $2.05 \AA$, with a standard deviation of $0.11 \AA .{ }^{41}$ In the Amber $14 \mathrm{SB}$ force field, sulfur atoms in SS bonds are held together with a harmonic potential at an equilibrium bond distance of $2.038 \AA$. This enforces a narrow distribution of bond distances centered at this value in our simulations for sulfurs connected in SS bonds. Once SS bonds are broken, sulfur atoms interact through a Leonard Jones potential with a minimum in the energy well at a value of $4.00 \AA$. Consequently, most cleaved pairs of $S \gamma$ atoms maintain a range between 4 to $5 \AA$, suggesting that the $S \gamma$ atoms remain in close enough proximity to possibly reform the SS bond in the case it is broken. Therefore, it was not surprising to observe an increase in the separation distance of $\mathrm{S} \gamma$ atoms to the range of 4 to $5 \AA$ upon cleavage, as seen in the majority of distances for both $\mathrm{IgG}$ and BSA.

It is important to note, however, that the atomistic simulations presented here do not allow reformation of covalent bonds. Pairs of sulfur atoms that separate significantly upon cleavage of the SS bond have a much lower probability of bond reformation. Because $S \gamma$ atoms of cleaved SS bonds maintain an average distance of $4.1 \pm 0.5 \AA$, a significant separation was defined as $>6 \AA$ (roughly 4 standard deviations). ${ }^{19}$ Thus, in the atomistic simulations, when the separation distance of S $\gamma$ atoms increased past $6 \AA$ it was likely that there was not sufficient intermolecular interaction energy and it was probable that the SS bond existed to maintain local protein structure. In contrast, when pairs of $S \gamma$ atoms remained in close proximity after breaking a SS bond, the importance the SS bond played in maintaining local structure may have been supplemented with other protein-protein interactions. In each protein, separation distances between $\mathrm{S} \gamma$ atoms of SS bonds were calculated. Separation distances in simulation runs with all native SS bonds connected were compared to distances in simulations runs with all native SS bonds 
disconnected. As with native non-bonded cysteine residues, $\mathrm{S} \gamma$ atoms in cysteine residues of proteins with no SS bonds were changed to a sulfhydryl group.

In IgG, separation distances for all connected SS bonds (simulation run 1) maintained an average value of $2.0 \AA$, with a standard deviation less than $0.1 \AA$. In $\operatorname{IgG}$ without native SS bonds (simulation run 2), distances between all pairs of S $\gamma$ atoms from cleaved intra-chain SS bonds ranged between 3 to $5 \AA$, with an average value of $3.8 \pm 0.3 \AA$. Given the Leonard Jones potential, this suggested possibility of bond reformation. In contrast, distances between pairs of $\mathrm{S} \gamma$ atoms from cleaved inter-chain SS bonds were significantly higher, averaging 14.2 $\pm 3.4 \AA$. Table 4 details separation distances for $S \gamma$ atoms in $\operatorname{IgG}$ simulation run 2 (without SS bonds) for all 15 sulfhydryl pairs. Figure 6 presents the separation distance between S $\gamma$ atoms for Cys236H-Cys236K, an inter-chain SS bond of the hinge region, in IgG simulation run 1 (with SS bonds) and simulation run 2 (without SS bonds). Separation distances in the remaining two inter-chain SS bonds (Cys230H-Cys215L and Cys230K-Cys215M) for simulation run 2 are also shown. Additionally, the separation distance between $\mathrm{S} \gamma$ atoms of simulation run 2 for Cys22H-Cys96H, an intra-chain SS bond in $\mathrm{F}_{\mathrm{ab}} 1$, is presented for comparison.

It is important to note that all intra-chain SS bonds in the protein with native SS bonds cleaved maintained a narrow separation distance distribution centered at $3.8 \AA$, indicating a possibility to reform the SS bond (see Table 4). Therefore, Cys22H-Cys96H was selected as a representative of intra-chain SS bonds to serve as a comparison in Figure 6. Similarly, all S $\gamma$ pair atom distances in IgG with SS bonds maintained a narrow separation distribution centered at $2.0 \AA$; therefore, Cys $236 \mathrm{H}-\mathrm{Cys} 236 \mathrm{~K}$ was selected to represent a bonded $\mathrm{S}_{\gamma}$ pair for comparison in Figure 6. As expected, distances between $\mathrm{S} \gamma$ atoms of Cys $236 \mathrm{H}-\mathrm{Cys} 236 \mathrm{~K}$ in IgG simulation run 1 (with SS bonds) maintained an average separation distance of $2.0 \AA$. In IgG simulation run 2 (without SS bonds), distances between $\mathrm{S} \gamma$ atoms of Cys22H-Cys96H averaged $3.8 \AA$. Separation distances for S $\gamma$ atoms of Cys $236 \mathrm{H}-\mathrm{Cys} 236 \mathrm{~K}$ were significantly higher, averaging at a value of $10.3 \AA$.

\begin{tabular}{|c|c|c|c|}
\hline Sulfhydryl Pair & Inter-Chain SS Bond & Average $(\AA)$ & Standard deviation $(\AA)$ \\
\hline $22 \mathrm{H}-96 \mathrm{H}$ & No & 3.8 & 0.3 \\
\hline 23L-89L & No & 3.9 & 0.3 \\
\hline $154 \mathrm{H}-210 \mathrm{H}$ & No & 3.9 & 0.4 \\
\hline 135L-195L & No & 3.8 & 0.4 \\
\hline $22 \mathrm{~K}-96 \mathrm{~K}$ & No & 3.8 & 0.3 \\
\hline 23M-89M & No & 3.9 & 0.3 \\
\hline $154 \mathrm{~K}-210 \mathrm{~K}$ & No & 3.8 & 0.3 \\
\hline 135M-195M & No & 3.8 & 0.4 \\
\hline 271H-331H & No & 3.9 & 0.4 \\
\hline $377 \mathrm{H}-435 \mathrm{H}$ & No & 3.8 & 0.4 \\
\hline 271K-331K & No & 3.9 & 0.3 \\
\hline 377K-435K & No & 3.8 & 0.3 \\
\hline $230 \mathrm{H}-215 \mathrm{~L}$ & Yes & 16.4 & 1.7 \\
\hline $230 \mathrm{~K}-215 \mathrm{M}$ & Yes & 15.8 & 2.6 \\
\hline $236 \mathrm{H}-236 \mathrm{~K}$ & Yes & 10.3 & 0.9 \\
\hline
\end{tabular}

Table 4. Summary of separation distances between $S \gamma$ atoms of all 15 sulfhydryl pairs of $\operatorname{IgG}$ without SS bonds (simulation run 2), including identification of inter-chain or intra-chain SS bonds. All intra-chain S $\gamma$ atoms maintained a distance suggestive of possible bond reformation ( 3 to $5 \AA$ ), while inter-chain S $\gamma$ atoms deviated significantly, 


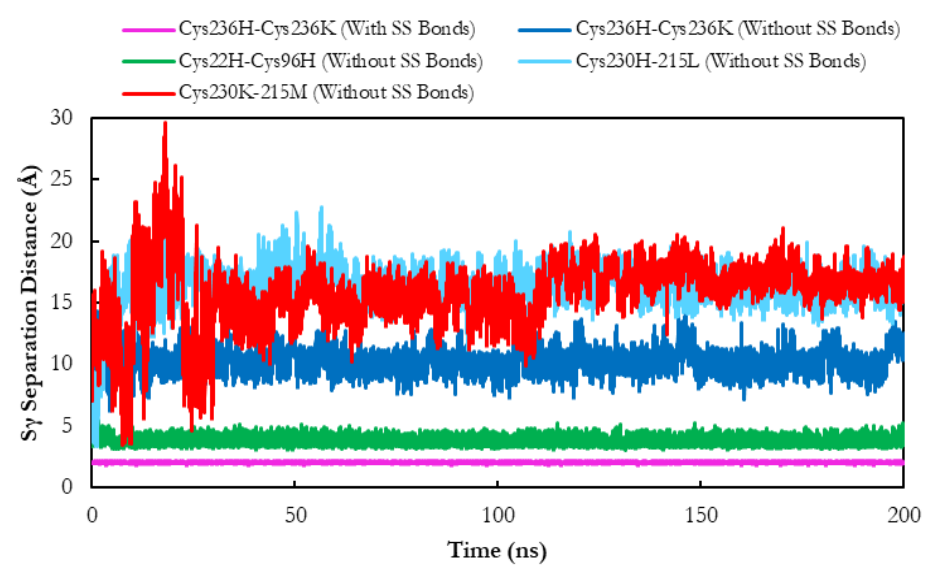

Figure 6. Separation distances between $S \gamma$ atoms of selected sulfhydryl pairs for $\operatorname{IgG}$ with SS Bonds (simulation run 1) and without SS Bonds (simulation run 2). Separation distances are presented for Cys236H-Cys236K with SS Bonds (simulation run 1, in pink), Cys236H-Cys $236 \mathrm{~K}$ without SS Bonds (simulation run 2, in blue), Cys22H-Cys96H without SS Bonds (simulation run 2, in green), Cys230H-Cys215L without SS bonds (simulation run 2, in teal) and Cys230K-Cys215M without SS bonds (simulation run 2, in red).

As discussed, Sy atoms of intra-chain SS bonds in IgG without SS bonds sampled distances ranging between 3 to $5 \AA$. The previously developed prescreening methodology yielded three inter-chain $S \gamma$ atom pairs with significant distance separations. In addition to notable separation distances in the hinge Cys236H-Cys236K bond, the two remaining inter-chain SS bonds also deviated significantly (>6 $\AA$ ). Cys230H-Cys215L and Cys230K-Cys215M are found at symmetric junctions of a heavy chain and light chain in $\mathrm{F}_{\mathrm{ab}} 1$ and $\mathrm{F}_{\mathrm{ab}} 2$, respectively, of $\operatorname{IgG}$. Because intra-chain SS bonds of $\operatorname{Ig} \mathrm{G}$ are buried in a stable secondary structure of $\beta$ (beta) sheets, it is hypothesized that cleaved bonds were supplemented by other local interactions. In inter-chain SS bonds, the large variability (range of 3 to $30 \AA$ ) and significantly increased values of $S \gamma$ atom separation distances suggested these SS bonds maintain local protein structure and possibly control the dynamic movement of $\mathrm{F}_{\mathrm{ab}}$ domains. Previous work detailing the segmental motion and high degree of flexibility imparted by the hinge region, and therefore suggesting structural importance, prompted selection of Cys236H-Cys236K for cleavage.

S $\gamma$ atom pair separation distances were also calculated for BSA. Similar to IgG, S $\gamma$ atom pairs of BSA with native SS bonds connected maintained an average distance of $2.0 \AA$, with a standard deviation less than $0.1 \AA$, while most $S \gamma$ atom pairs in BSA with all native SS bonds disconnected sampled a range of distances from 3 to $5 \AA$, with an average value of $4.5 \pm 0.6 \AA$. Distances between $S \gamma$ atom pairs of Cys167-Cys176 and Cys123-Cys168 deviated significantly (>6 $\AA$ ), averaging $7.0 \pm 2 \AA$ and $5.0 \pm 1.5 \AA$, respectively. S $\gamma$ atom pairs of Cys475-Cys486, Cys513-Cys558, and Cys315-Cys360 also showed significant separation distances, fluctuating between 3 to $11 \AA$. Cys167-Cys176, comprised of a sulfhydryl pair with a noticeably high S $\gamma$ separation distance and a large degree of fluctuation, is part of a double SS bond with Cys123-Cys168. From the results of the prescreening approach, as well as a homologous double SS bond with high S $\gamma$ separation found in our previous study of HSA, this double SS bond was selected. Therefore, simulation runs 6-8 consisted of the native BSA structure with Cys123-Cys168 disconnected, Cys167-Cys176 disconnected, and both Cys123-Cys168 and Cys167-Cys176 disconnected, respectively.

Figure 7 presents a comparison between S $\gamma$ atom pair separation distances in Cys167-Cys176 in BSA with SS bonds (simulation run 4) and without SS bonds (simulation run 5). Additionally, S $\gamma$ atom pair separation distances in Cys199-Cys245 in BSA without SS bonds (simulation run 5) is presented. All S $\gamma$ atom pairs with no significant $(<6 \AA)$ separation distances maintained a narrow separation distance distribution centered at $4.5 \AA$, indicating a strong possibility of bond reformation given the Leonard Jones potential. Therefore, Cys199-Cys245 was selected to represent these SS bonds to serve as a comparison in Figure 7. Similarly, all S $\gamma$ pair atom distances in BSA with SS bonds maintained a narrow distance distribution centered at $2.0 \AA$; therefore, Cys167-

Cys176 was selected as a bonded S $\gamma$ pair for comparison in Figure 7. As expected, distances between S $\gamma$ atoms of Cys167-Cys176 in BSA simulation run 4 (with SS bonds) maintained an average separation distance of $2.0 \AA$. In BSA simulation run 5 (without SS bonds), distances between $S \gamma$ atoms of Cys199-Cys245 averaged $4.9 \AA$. While separation distances exceeded $6 \AA$ in several frames of the trajectory, $S \gamma$ atoms of Cys199-Cys 245 remained in a separation range of 4 to $6 \AA$ for the vast majority of simulation time, supporting the possibility of bond reformation. S $\gamma$ atoms of Cys167-Cys176 averaged a noticeably higher average value of $7.3 \AA$ and sampled a range of distances from 4 to $13 \AA$. 


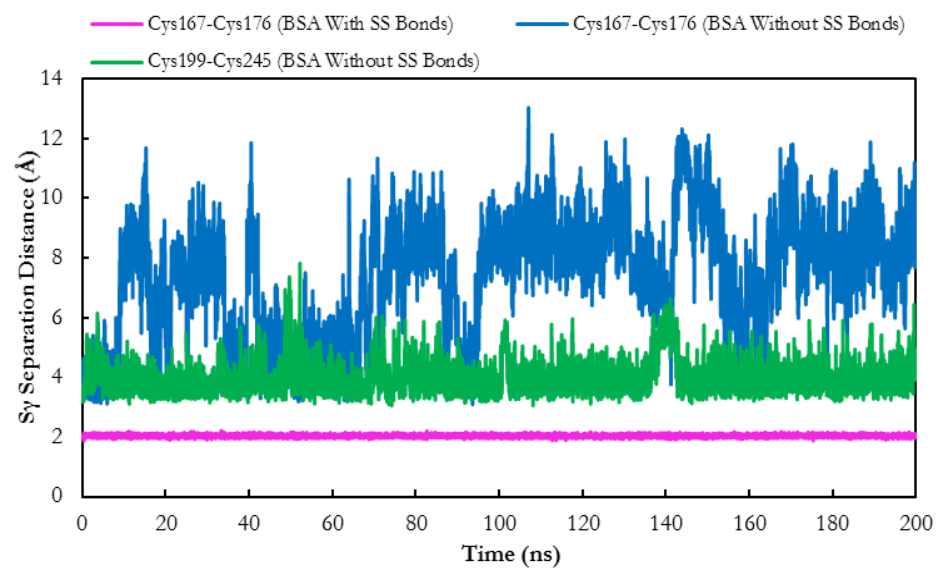

Figure 7. Separation distances between $S \gamma$ atoms of selected sulfhydryl pairs for BSA with SS Bonds (simulation run 4) and without SS Bonds (simulation run 5). Separation distances are presented for Cys167-Cys176 with SS Bonds (simulation run 4, in pink), Cys167-Cys176 without SS Bonds (simulation run 5, in blue), and Cys199-Cys245 without SS Bonds (simulation run 5, in green).

Figure 8a shows separation distances between S $\gamma$ atoms in Cys123-Cys168 for simulation runs 4 (with SS bonds), 6 (Cys123Cys168 disconnected), and 8 (both Cys123-Cys168 and Cys167-176 disconnected). In simulation runs 6 and 8, S $\gamma$ atoms of Cys123-Cys168 maintained a similar separation distance, ranging between 4.5 to $6 \AA$. Figure $8 \mathbf{b}$ shows separation distance of $\mathrm{S} \gamma$ atoms in Cys167-Cys176 for simulation runs 4 (with SS bonds), 7 (Cys167-Cys176 disconnected) and 8 (both Cys123-Cys168 and Cys167-176 disconnected). In contrast to Cys123-Cys168 S $\gamma$ atoms, Cys167-Cys176 S $\gamma$ atoms were able to separate to a greater extent when both Cys123-Cys168 and Cys167-Cys176 were broken in the double SS bond, as evidenced in a greater average separation distance of $8 \AA$ in simulation run 8 compared to a distance of $5 \AA$ in simulation run 7 . Figure 9 summarizes the average separation distances of $S \gamma$ atoms in Cys123-Cys168 and Cys167-Cys176 when only one or both of the double SS bonds were broken. The average separation distance of Cys167-Cys176 shown in Figure 9 was calculated from data gathered after 100 ns, where clear separation was first observed.
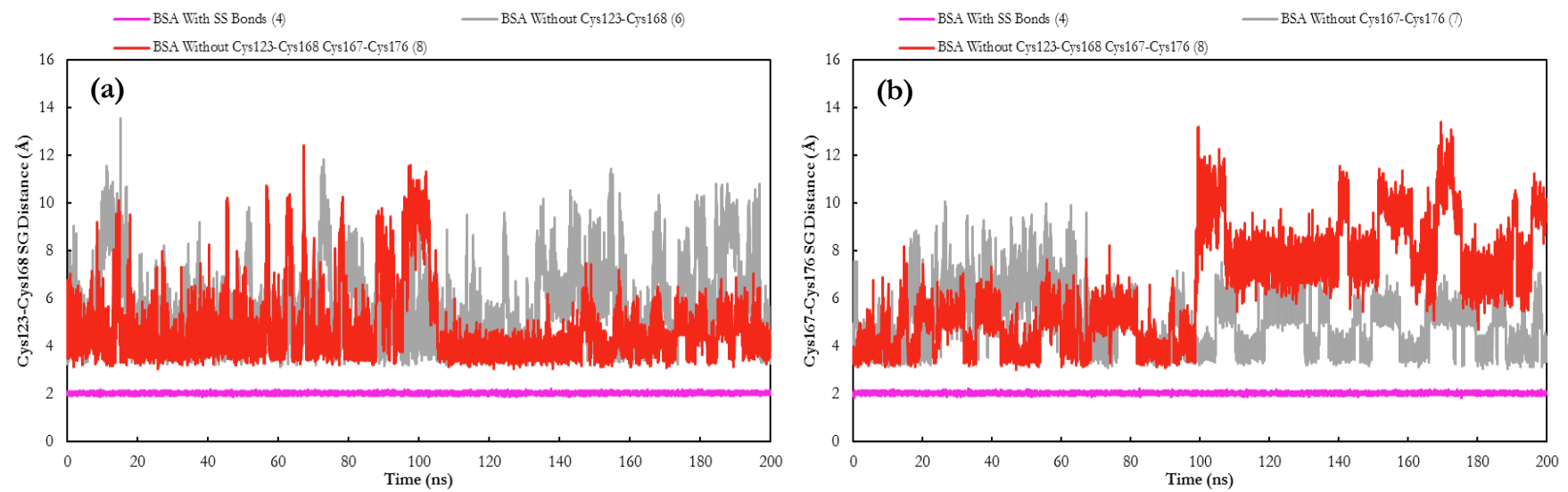

Figure 8. Separation distances between Sy atoms of Cys123-Cys168 (a) and Cys167-Cys176 (b) for BSA simulation runs 4, 6, 7, and 8. Separation distances are presented for BSA with SS Bonds (simulation run 4, in pink), BSA without one SS Bond (simulation runs 6 and 7, in gray), and BSA without both SS Bonds (simulation run 8 , in red). 


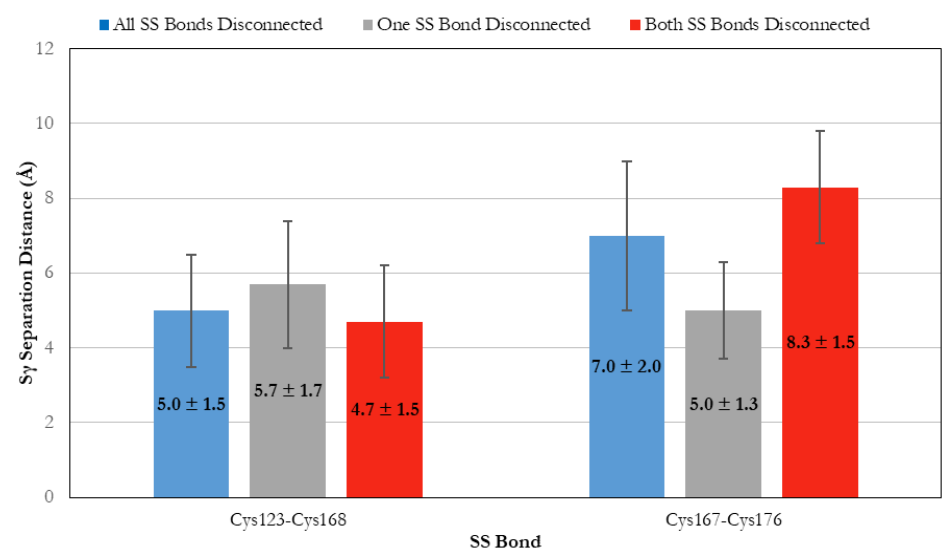

Figure 9. Summary of separation distances between $S \gamma$ atoms of Cys123-Cys168 and Cys167-Cys176 for BSA simulation runs 5-8. Separation distances are presented for BSA without SS Bonds (simulation run 5, in blue), BSA without one SS Bond (simulation runs 6 and 7, in gray), and BSA without both SS Bonds (simulation run 8 , in red).

All 17 SS bonds in BSA are intra-domain and buried in the $\alpha$ helices of the protein structure. Results shown in Figures 7-9 show that Cys123-Cys168 and Cys167-Cys176 form a double SS bond, in subdomain IB, with significant separation distances in S $\gamma$ atoms upon cleavage, suggesting contributions to protein structure stability. Lower separation distances in $\mathrm{S} \gamma$ atoms of Cys123Cys168 in comparison to Cys167-Cys176 also suggests that intermolecular forces may have contributed more to local protein structure stabilization around Cys123-Cys168, while Cys167-Cys176 may have been responsible in maintaining secondary or tertiary structure of BSA. Homologous SS bonds were identified in previous work on HSA, in which Cys124-Cys169 and Cys168177 were identified as a double SS bond in IB. ${ }^{19}$ These SS bonds were found to provide stability to local structure and dynamics.

\section{Structural Changes}

To investigate structural changes in the proteins, vector analysis was used to track coordinates of the center of mass of each protein domain, relative to the origin, over the entire trajectory of all simulation runs. Separation distances (Euclidean distance) between domains over time were then calculated using the coordinates. In IgG, vector coordinates of the center of mass of the $\mathrm{F}_{\mathrm{ab}} 1, \mathrm{~F}_{\mathrm{ab}} 2$, and $\mathrm{F}_{\mathrm{c}}$ domains were calculated. In BSA, vector coordinates of the center of mass of the I, II, and III domains were calculated. Pairwise separation distances were computed. After vector analysis, VMD was used visualize conformational changes in IgG and BSA after cleavage of SS bonds. For reference, Figure 10 shows a visualization of IgG and BSA with their respective domain centers of mass highlighted and associated distances displayed.
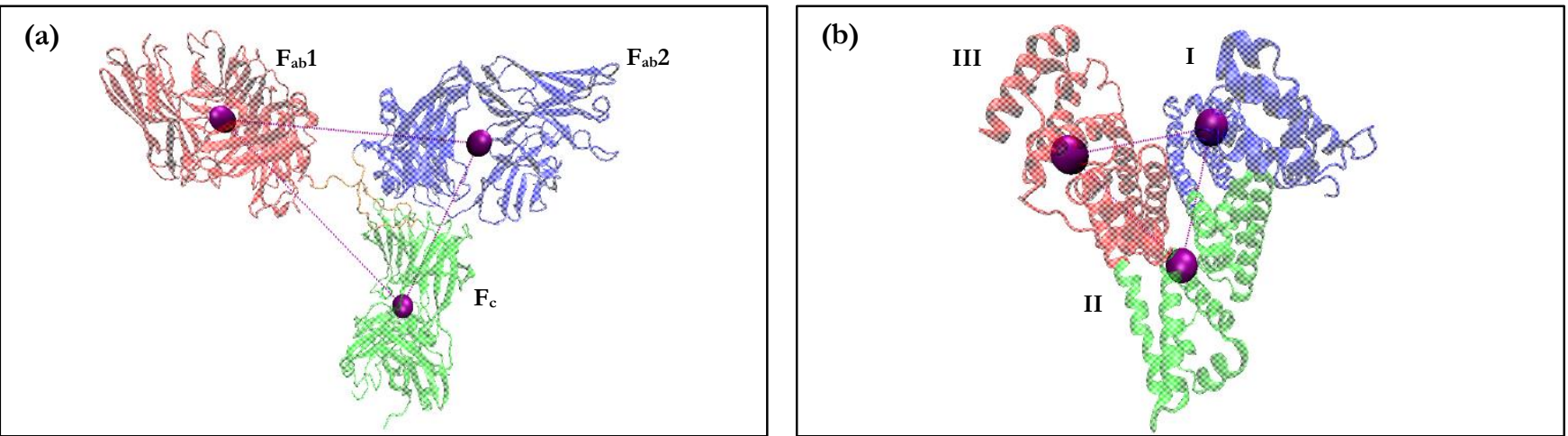

Figure 10. Cartoon representation of $\operatorname{IgG}$ (a) depicts centers of mass as purple van der Waals (vdW) spheres and associated distances as dashed purple lines. Regions are represented as: $\mathrm{F}_{\mathrm{ab}} 1$ (red), $\mathrm{F}_{\mathrm{ab}} 2$ (blue), $\mathrm{F}_{\mathrm{c}}$ (green), and hinge (orange). Cartoon representation of BSA (b) depicts centers of mass as purple van der Waals (vdW) spheres and associated distances as dashed purple lines. Domains are represented as: I (blue), II (green), and III (red). The cartoons were created using Visual Molecular Dynamics. ${ }^{26}$

Distances between domains $F_{a b} 1$ and $F_{c}$, as well as between $F_{a b} 2$ and $F_{c}$, remained stable across all simulation runs (1-3), with little deviation between $\operatorname{IgG}$ systems. Figure 11 details a notable observation in the distance between the centers of mass for $\mathrm{F}_{\mathrm{ab}} 1$ and $\mathrm{F}_{\mathrm{ab}} 2$. For simulation runs 1 and 3, the distances between the two domains remained stable over evolution of 200 ns with average 
values of $81.5 \pm 2.25 \AA$ and $79.9 \pm 2.36 \AA$, respectively. The separation distance in simulation run 2 maintained an average value of $75.6 \pm 4.94 \AA$ during the entire simulation time. The distance increased for the first $\sim 15 \mathrm{~ns}$, then decreased significantly for $\sim 45 \mathrm{~ns}$, where the distance then remained at a stable $72.2 \pm 1.26 \AA$ for $\sim 20 \mathrm{~ns}$, and then increased again to remain at a stable $73.8 \pm 1.24 \AA$ for the remainder of simulation time. After $\sim 30 \mathrm{~ns}$, the separation distance between the two domains for simulation run 2 was consistently lower than those for simulation runs 1 and 3, suggesting a contraction of the protein structure. Separation distances in simulation runs 1 and 3 were not significantly different (deviation of average values for equivalent sample sizes overlapped), indicating that removal of the Cys236H-Cys236K SS bond was not responsible for the contraction.

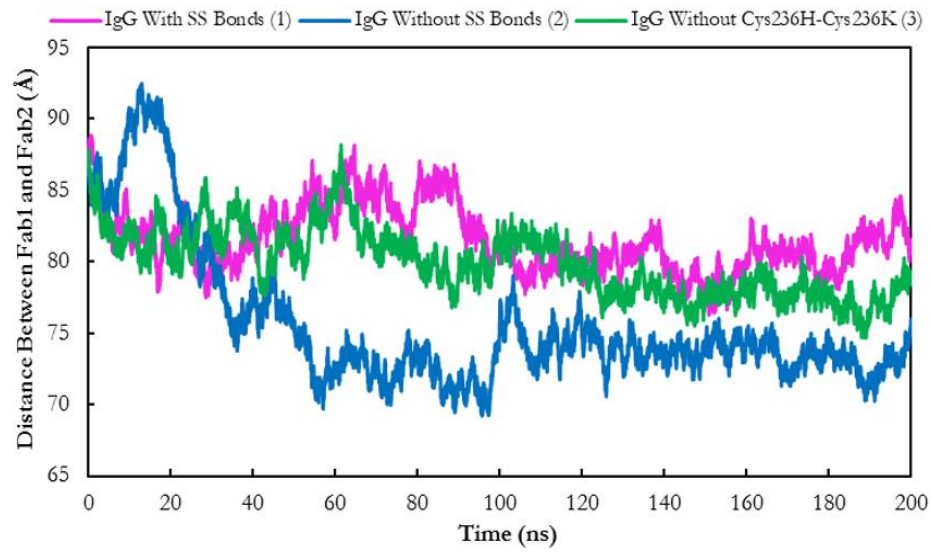

Figure 11. Distance between centers of mass of $\mathrm{F}_{\mathrm{ab}} 1$ and $\mathrm{F}_{\mathrm{ab}} 2$ domains for $\mathrm{IgG}$ simulations 1-3. Distances are presented for IgG with SS Bonds (simulation run 1 , pink), IgG without SS Bonds (simulation run 2, blue), and IgG without Cys236H-Cys236K (simulation run 3, green).

Vector analysis of IgG was followed by use of VMD to visualize structural changes. The hinge region itself lacks secondary structure; therefore, absence of stabilizing forces allows for the perceived segmental motion and flexibility. With this knowledge, visualization of local structure about the hinge region would not provide relevant observations. Visualization of domains displayed evidence of the contraction seen in Figure 11. Figure 12 shows snapshots of IgG during simulation run 2 (without SS bonds) at 20 ns (Figure 12a) and at 120 ns (Figure 12b). Relative to the protein structure in early stages of the trajectory, distance between $\mathrm{F}_{\mathrm{ab}} 1$ and $\mathrm{F}_{\mathrm{ab}} 2$ domains of $\mathrm{IgG}$ decreased. This contraction was seen in the IgG molecule, and this state persisted for the remainder of simulation time. When trajectories of simulation run 1 and 3 (with SS bonds and without Cys236H-Cys236K) were visualized, no similar contraction was seen. Again, this suggested that cleavage of Cys236H-Cys236K was not directly responsible for the observed conformational change.
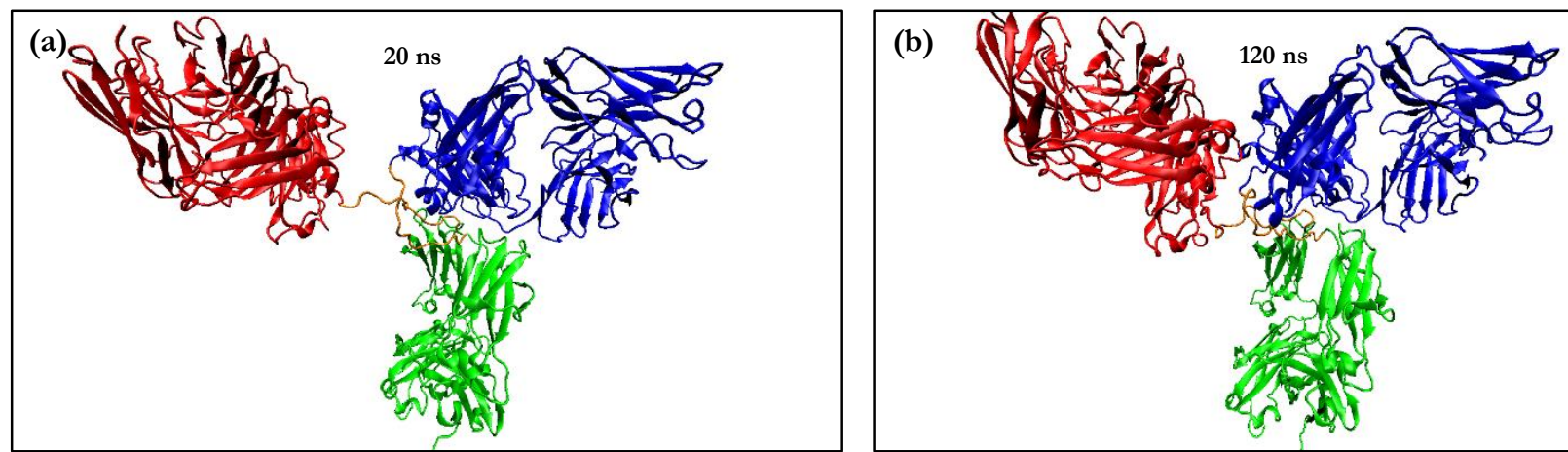

Figure 12. Cartoon representation of IgG at $20 \mathrm{~ns}$ (a) and $120 \mathrm{~ns}$ (b). Regions are represented as: $\mathrm{F}_{\mathrm{ab}} 1$ (red), $\mathrm{F}_{\mathrm{ab}} 2$ (blue), $\mathrm{F}_{\mathrm{c}}$ (green), and hinge (orange). Contraction of protein is seen with the decrease in distance between $\mathrm{F}_{\mathrm{ab}} 1$ and $\mathrm{F}_{\mathrm{ab}} 2$ domains. The cartoons were created using Visual Molecular Dynamics. ${ }^{26}$

In BSA, pairwise distances between domains I, II, and III were computed. Distances remained stable across all simulations runs (4-8), with little deviation between BSA systems. Figure 13 shows distances for domains I-II, I-III, and II-III over 200 ns simulation time. Domains I and II maintained an average distance of $32 \pm 0.4 \AA$, and domains I and III had an average distance of $35 \pm 0.7 \AA$ for all simulation runs. For domains II and III, distances fluctuated around $35 \pm 0.6 \AA$. In all cases, distances were not significantly different across simulation runs. 

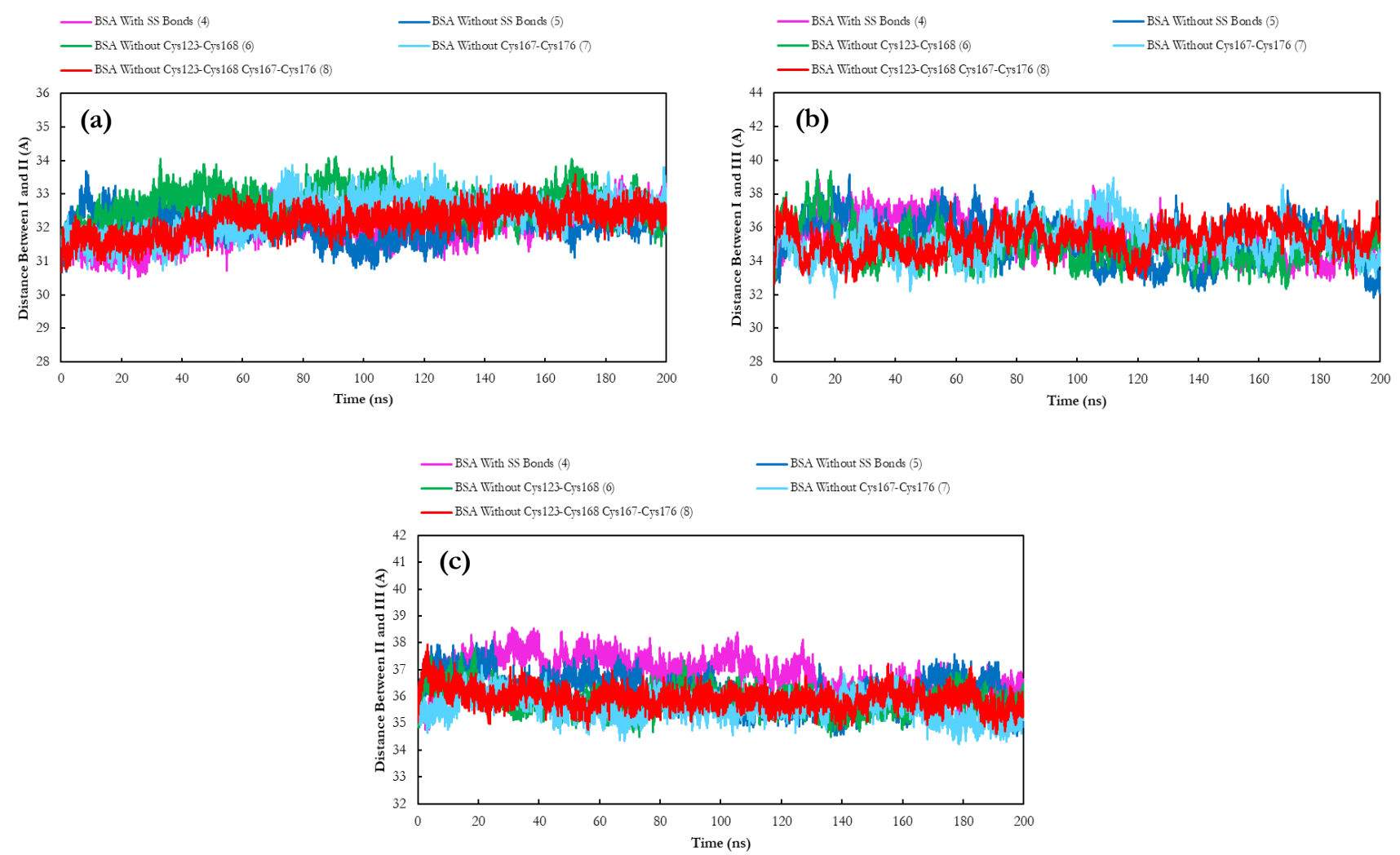

Figure 13. Distance between centers of mass of I, II, and III domains for BSA simulations 4-8. Distances for I-II (a), I-III (b), and II-III (c) are presented for BSA with SS Bonds (simulation run 4, in pink), without SS Bonds (simulation run 5, in blue), without Cys123-Cys168 (simulation run 6, in green), without Cys167Cys176 (simulation run 7, in teal), and without Cys123-Cys168 and Cys167-Cys176 (simulation run 8, in red).

All SS bonds of BSA are buried in the $\alpha$ helices of the protein structure. The protein's globular nature imparts little to no flexibility. Therefore, large deviations in distances between domains of BSA were not expected. Upon application of the prescreening method, S $\gamma$ atom pairs of the double SS bond (Cys123-Cys168 and Cys167-Cys176) of BSA with all SS bonds cleaved separated significantly; this suggested possible changes in local protein structure. Three $\alpha$ helices were involved in the double SS bond. These helices, identified as A, B, and C, were comprised of residues 119-145, residues 150-172, and residues 175 to 195, respectively. Cys123-Cys168 was between helices A and B, and Cys167-Cys176 was between helices B and C. Figure 14 shows snapshots of those $\alpha$ helices in BSA during simulation run 5 (without SS bonds) at 10 ns (Figure 14a) and 180 ns (Figure 14b). A local perturbation in secondary structure was seen in helix $C$ during the course of the simulation run, as identified in the figure.
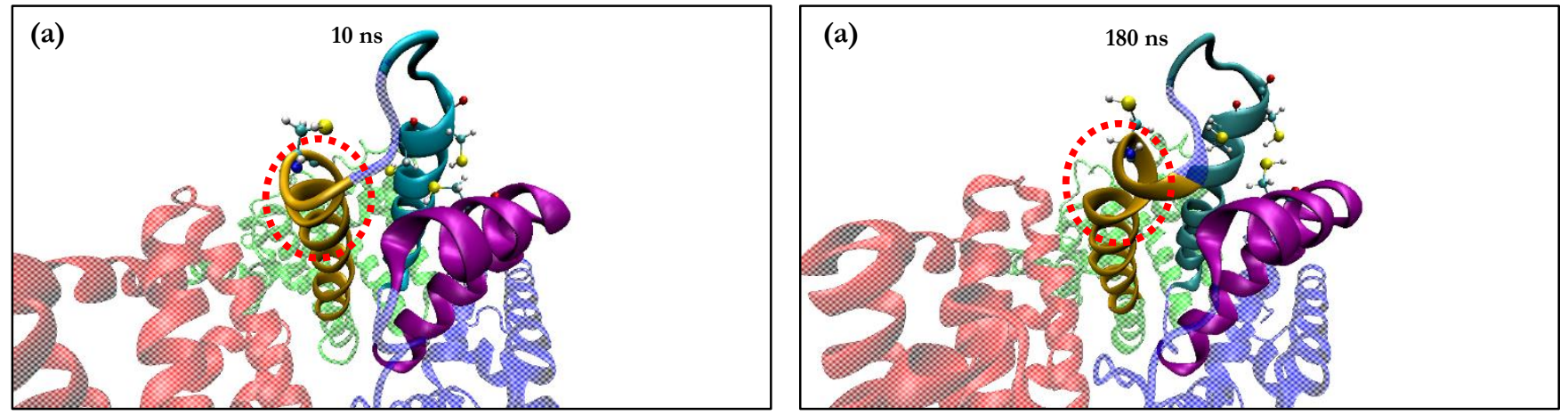

Figure 14. Cartoon representation of BSA at $10 \mathrm{~ns}$ (a) and $180 \mathrm{~ns}$ (b). Domains are represented as: I (blue), II (green), and III (red). Helices are represented as: A (purple), B (cyan), and C (orange). SS bonds are represented as ball-and-stick. Local perturbation in secondary structure seen in helix C, as identified by the red circle. The cartoons were created using Visual Molecular Dynamics. ${ }^{26}$ 
Because of the distinct protein structures of $\mathrm{IgG}$ and BSA, the role and contributions of SS bonds to overall structure maintenance were inherently dissimilar. As discussed, application of the prescreening methodology showed that $\mathrm{S} \gamma$ atom pairs of inter-chain SS bonds in IgG deviated significantly, while pairs in intra-chain SS bonds did not. Unlike intra-chain SS bonds, interchain bonds of $\mathrm{IgG}$ are not buried in $\beta$ sheets; therefore, secondary structure cannot supplement interactions in these local residues. Specifically, the selected SS bond for cleavage, Cys236H-Cys236K, was between two heavy chains in the hinge region. Thus, analysis of structural change in IgG was made at the domain level, and a contraction of the protein was observed. In contrast, all SS bonds of BSA are intra-domain and buried in $\alpha$ helices. For S $\gamma$ atom pairs with significant deviations, secondary structure was present, but did not stabilize local interactions. This suggested more local structural changes, and perturbations were observed at the $\alpha$ helix level. In the case of IgG, the selected SS bond (Cys236H-Cys236K) did not reproduce the contraction, but separation distance data does suggest that one or more of the three inter-chain SS bonds contributed to structural changes in simulation run 2 (without SS bonds). In both cases, the prescreening method showed that analyzing simulations of proteins with all native SS bonds cleaved and calculating S $\gamma$ atom pair separation distances successfully identified specific SS bonds critical to structural stability.

\section{CONCLUSIONS}

In this study, a prescreening methodology introduced in previous work by our lab to identify potentially significant SS bonds was extended to study their role in the stabilization of IgG and BSA. IgG was selected for its importance in current monoclonal antibody therapies, high density of SS bonds, and novel structure. BSA maintains structural homology with HSA, the protein studied in our previous work, and therefore provided an avenue through which the relationship between structure and function could be considered. Atomistic MD simulations of IgG and BSA were performed for a total simulation time of $1.6 \mu$ s. For each protein, SS bond distances for independent simulation runs with native SS bonds cleaved were calculated and analyzed. The relative importance of inter, intra, and double SS bonds was evaluated with respect to which sulfur atom pairs deviated significantly from the average disulfide bond distance $(2.05 \AA)$.

In IgG, RMSD analyses of three independent simulation runs showed longer equilibration time scales for runs without all native SS bonds, indicating the likelihood of structural changes. Only S $\gamma$ atoms of inter-chain SS bonds, such as Cys236H-Cys236K, showed significant $(>6 \AA)$ deviations in distances, while those of intra-chain SS bonds remained in a stable range of 3 to $6 \AA$. The high degree of $\beta$ sheet secondary structure likely stabilized intra-chain SS bonds. Thus, intra-chain SS bonds of IgG may provide redundant interactions for conformational stability or play a role in protein function. Vector analysis and visualization showed a contraction of the protein between $\mathrm{F}_{\mathrm{ab}} 1$ and $\mathrm{F}_{\mathrm{ab}} 2$ domains, but cleavage of only Cys236H-Cys236K showed it was not solely responsible. This does not discount the possibility of one or more inter-chain SS bonds contributing to these protein dynamics. In BSA, all SS bonds are intra-domain and supplemented by secondary structure of $\alpha$ helices. S $\gamma$ atoms of several bonds, including a double SS bond (Cys123-Cys168 and Cys167-Cys176), showed significant deviations. Previous work on HSA showed a homologous double SS bond, whose $\mathrm{S} \gamma$ atoms also deviated significantly upon cleavage and contributed to structural stability. Analysis and visualization showed that Cys167-Cys176 may noticeably contribute to maintaining local structure.

This work demonstrated the efficacy of a previously established prescreening methodology in the use of atomistic simulations to identify structurally significant SS bonds. In simulations of both IgG, a large, Y-shaped protein, and BSA, a small, globular protein similar to HSA, use of the methodology effectively identified pairs of $\mathrm{S} \gamma$ atoms with significant changes in distances. Application of the prescreening method was achieved; future work may include more rigorous analysis of protein dynamics shown in this study (local RMSDs, secondary structure analysis, correlated motions, etc.). The absence of select disulfide bonds in proteins has been identified to lead to susceptibility to protein aggregation. These simulations provide information on the effects of specific SS bonds in conformational stability of both proteins, and the discussed method provides a predictive power in identifying these bonds, which may be critical in protein stability and/or aggregation phenomena. Additionally, prediction of SS bonds relevant to protein stabilization can be subsequently tested experimentally (selective reduction), providing a practical approach for faster screening in SS bond analysis. Results of this prompt more comprehensive insight into the role of SS bonds and changes in protein dynamics following their absence.

\section{ACKNOWLEDGEMENTS}

The authors thank Dr. Ping Lin and Dr. Maria Monica Castellanos for assistance in the modeling and reconstruction of the IgG structure. They also acknowledge the computational resources (HiPerGator 2.0) provided by University of Florida Research Computing and the funding provided by National Science Foundation (grant ACI-1613155). 


\section{REFERENCES}

1. Berg, J. M., Tymoczko, J. L., and Stryer, L. (2002) Biochemistry $5^{\text {th }}$ ed., 84-137, W. H. Freeman, New York.

2. Bulaj, G. (2005) Formation of disulfide bonds in proteins and peptides, Biotechnol. Adv. 23, 87-92. doi:10.1016/j.biotechadv.2004.09.002.

3. Trivedi, M. V., Laurence, J. S., and Siahaan, T. J. (2009) The role of thiols and disulfides in protein chemical and physical stability, Curr. Protein Pept. Sci. 10(6), 614-625. doi:10.2174/138920309789630534.

4. Creighton T. E. (1997) Protein folding coupled to disulphide bond formation, Biol. Chem. 378(8), 731-744. doi:10.1515/bchm.1997.378.8.731.

5. Ross, C. A., and Poirier, M. A. (2004) Protein aggregation and neurodegenerative disease, Nat. Med. 10, S10-S17. doi:10.1038/nm1066.

6. Trottier Y., Lutz Y., Stevanin G., Imbert G., Devys D., Cancel G., Saudou F., Weber C., David G., Tora L., Agid Y., Brice A., and Mandel J. L. (1995) Polyglutamine expansion as a pathological epitope in Huntington's disease and four dominant cerebellar ataxias, Nature 378, 403-406. doi:10.1038/378403a0.

7. Voisine C., Pedersen J. S., and Morimoto R. I. (2010) Chaperone networks: Tipping the balance in protein folding diseases, Neurobiol. Dis. 40(1), 12-20. doi:10.1016/j.nbd.2010.05.00.

8. Williams A., Jahreiss L., Sarkar S., Saiki S., Menzies F. M., Ravikumar B., and Rubinsztein D. C. (2006) Aggregate-prone proteins are cleared from the cytosol by autophagy: Therapeutic implications, Curr. Top. Dev. Biol. 76, 89-101. doi:10.1016/S0070-2153(06)76003-3.

9. Cho S. S., Levy Y., Onuchic J. N., and Wolynes P. G. (2005) Overcoming residual frustration in domain-swapping: the roles of disulfide bonds in dimerization and aggregation, Phys. Biol. 2, S44-S55. doi:10.1088/1478-3975/2/2/S05.

10. Welker E., Wedemeyer W. J., and Scheraga H. A. (2001) A role for intermolecular disulfide bonds in prion diseases? Proc. Natl. Acad. Sci. USA 98, 4334-4336. doi:10.1073/pnas.071066598.

11. Liu, H., and May, K. (2012) Disulfide bond structures of IgG molecules: Structural variations, chemical modifications and possible impacts to stability and biological function, $M A b s .4(1), 17-23$. doi:10.4161/mabs.4.1.18347.

12. Yang, M., Dutta, C., and Tiwari, A. (2015) Disulfide-bond scrambling promotes amorphous aggregates in lysozyme and bovine serum albumin, J. Phys. Chem. B 119(10), 3969-3981. doi:10.1021/acs.jpcb.5b00144.

13. Betz, S. F. (1993) Disulfide bonds and the stability of globular proteins, Protein Sci. 2(10), 1551-1558. doi:10.1002/pro.5560021002.

14. Borges, C. R., and Sherma, N. D. (2014) Techniques for the analysis of cysteine sulfhydryls and oxidative protein folding, Antioxid. Redox Signal. 21(3), 511-531. doi:10.1089/ars.2013.5559.

15. Buyong M. A., Xiong, J., Lubkowski, J., and Nussinov, R. (2000) Homology modeling and molecular dynamics simulations of lymphotactin, Protein Sci. 9(11), 2192-2199. doi:10.1110/ps.9.11.2192.

16. Godwin, A., Choi, J. W., Pedone, E. et al. (2007) Molecular dynamics simulations of proteins with chemically modified disulfide bonds, Theor. Chem. Acc. 117, 259. doi:10.1007/s00214-006-0134-0.

17. He J., Xu L., Zou Z., Ueyama N., Li H., Kato A., Jones G. W., and Song Y. (2013) Molecular dynamics simulation to investigate the impact of disulfide bond formation on conformational stability of chicken cystatin I66Q mutant, J Biomol. Struct. Dyn. 31(10), 1101-1110. doi:10.1080/07391102.2012.721498.

18. Zhang, L. (2017) Different dynamics and pathway of disulfide bonds reduction of two human defensins, a molecular dynamics simulation study, Proteins Struct. Funct. Bioinf. 85(4), 665-681. doi:10.1002/prot.25247.

19. Castellanos, M. M., and Colina, C. M. (2013) Molecular dynamics simulations of human serum albumin and role of disulfide bonds, J. Phys. Chem. B 117 (40), 11895-11905. doi:10.1021/jp402994r.

20. Vidarsson, G., Dekkers, G., and Rispens, T. (2014) IgG subclasses and allotypes: From structure to effector functions, Front. Immunol. 5, 520. doi:10.3389/fimmu.2014.00520.

21. Sandin, S., Ofverstedt, L. G., Wikstrom, A. C., Wrange, O., and Skoglund, U. (2004) Structure and flexibility of individual immunoglobulin G molecules in solution, Structure 12, 409-415. doi:10.1016/j.str.2004.02.011.

22. Berman, H. M., Westbrook, J., Feng, Z., Gilliland, G., Bhat, T. N., Weissig, H., Shindyalov, I. N., and Bourne, P. E. (2000) The Protein Data Bank, Nucleic Acids Res. 28, 235-242.

23. Saphire, E., Parren, P., Pantophlet, R., Zwick, M., Morris, G., Rudd, P., Dwek, R., Stanfield, R., Burton, D., and Wilson, I. (2001) Crystal structure of a neutralizing human IGG against HIV-1: a template for vaccine design, Science 293, $1155-1159$. doi:10.1126/science.1061692.

24. Chames, P., Van R. M., Weiss, E., and Baty, D. (2009) Therapeutic antibodies: successes, limitations and hopes for the future, Br. J. Pharmacol. 157(2), 220-233. doi:10.1111/j.1476-5381.2009.00190.x. 
25. Michaelsen, T. E., Brekke, O. H., Aase, A., Sandin, R. H., Bremnes, B., and Sandlie, I. (1994) One disulfide bond in front of the second heavy chain constant region is necessary and sufficient for effector functions of human $\operatorname{IgG} 3$ without a genetic hinge, Proc. Natl. Acad. Sci. USA 91(20), 9243-9247.

26. Humphrey, W., Dalke, A., and Schulten, K. (1996) VMD: Visual Molecular Dynamics, J. Molec. Graphics 14, 33-38. doi:10.1016/0263-7855(96)00018-5.

27. Merlot A. M., Kalinowski D. S., and Richardson D. R. (2014) Unraveling the mysteries of serum albumin-more than just a serum protein, Front. Physiol. 5, 299. doi:10.3389/fphys.2014.00299.

28. Gelamo E. L., and Tabak M. (2000) Spectroscopic studies on the interaction of bovine (BSA) and human (HSA) serum albumins with ionic surfactants, Spectrochim. Acta A Mol. Biomol. Spectrosc. 56, 2255-2271. doi:10.1016/S1386-1425(00)00313-9.

29. Majorek, K. A., Porebski, P. J., Dayal, A., Zimmerman, M. D., Jablonska, K., Stewart, A. J., and Minor, W. (2012) Structural and immunologic characterization of bovine, horse, and rabbit serum albumins, Mol. Immunol. 52(3-4), 174-182. doi:10.1016/j.molimm.2012.05.011.

30. Sugio, S., Kashima, A., Mochizuki, S., Noda, M., and Kobayashi, K. (1999) Crystal structure of human serum albumin at $2.5 \AA$ resolution, Protein Eng. 12, 439-446. doi:10.1093/protein/12.6.439.

31. Case, D. A., Betz, R. M., Cerutti, D. S., Cheatham, T. E., III, Darden, T. A., Duke, R. E., Giese, T. J., Gohlke, H., Goetz, A. W., Homeyer, N., Izadi, S., Janowski, P., Kaus, J., Kovalenko, A., Lee, T. S., LeGrand, S., Li, P., Lin, C., Luchko, T., Luo, R., Madej, B., Mermelstein, D., Merz, K. M., Monard, G., Nguyen, H., Nguyen, H. T., Omelyan, I., Onufriev, A., Roe, D. R., Roitberg, A., Sagui, C., Simmerling, C. L., Botello-Smith, W. M., Swails, J., Walker, R. C., Wang, J., Wolf, R. M., Wu, X., Xiao, L. and Kollman, P. A. (2016) AMBER 2016, University of California, San Francisco.

32. Salomon-Ferrer, R., Goetz, A., Poole, D., Le Grand, S., and Walker, R. (2013) Routine microsecond molecular dynamics simulations with AMBER on GPUs. 2. explicit solvent particle mesh Ewald, J. Chem. Theory Comput. 9, 3878-3888. doi:10.1021/ct400314y.

33. Maier, J. A., Martinez, C., Kasavajhala, K., Wickstrom, L., Hauser, K. E., and Simmerling, C. (2015) ff14SB: Improving the accuracy of protein side chain and backbone Pparameters from ff99SB, J. Chem. Theor. Comput. 11, 3696-3713. doi:10.1021/acs.jctc.5b00255.

34. Jorgensen, W. L., Chandrasekhar J., Madura J. D., Impey R. W., and Klein, M.L. (1983) Comparison of simple potential functions for simulating liquid water, J. Chem. Phys. 79, 926. doi:10.1063/1.445869.

35. Mahoney, M. W., and Jorgensen, W. L. (2000) A five-site model liquid water and the reproduction of the density anomaly by rigid, non-polarizable models, J. Chem. Phys. 112, 8910-8922. doi:10.1063/1.481505.

36. Crowley, M., Darden, T., Cheatham, T., III, and Deerfield, D., II. (1997) Adventures in improving the scaling and accuracy of a parallel molecular dynamics program, J. Supercomput. 11, 255-278. doi:10.1023/A:1007907925007.

37. Darden, T., York, D., Pedersen, L. (1993) Particle mesh ewald: An N · $\log (\mathrm{N})$ method for ewald sums in large systems, J. Chem. Phys. 98, 10089-10092. doi:10.1063/1.464397.

38. Essmann, U., Perera, L., Berkowitz, M. L., Darden, T., Lee, H., and Pedersen, L. G. (1995) A smooth particle mesh ewald method, J. Chem. Phys. 103, 8577-8593. doi:10.1063/1.470117.

39. Roe, D. R., Cheatham, T. III. (2013) PTRAJ and CPPTRAJ: Software for processing and analysis of molecular dynamics trajectory data, J. Chem. Theory Comput. 9, 3084-3095. doi:10.1021/ct400341p.

40. Fortunato, M. E., and Colina, C. M. (2014) Effects of galactosylation in immunoglobulin G from all-atom molecular dynamics simulations, J. Phys. Chem. B 118 (33), 9844-9851. doi:10.1021/jp504243e.

41. Bošnjak, I., Bojović, V., Šegvić-Bubić T., Bielen, A. (2014) Occurrence of protein disulfide bonds in different domains of life: a comparison of proteins from the Protein Data Bank, Protein Eng. Des. Sel. 27 (3), 65-72. doi.org/10.1093/protein/gzt063.

\section{ABOUT THE STUDENT AUTHORS}

Akshay Mathavan grew up in Davie in South Florida. He is currently an undergraduate junior student at the University of Florida, where he is pursuing a B. S. in Biomedical Engineering with minors in Mathematics and Computer Science. Akshay joined the Colina Research Group in the Winter of 2016. He plans to attend medical school and become an oncologist.

Akash Mathavan joined the Colina Research Group in December 2016. He grew up in Davie, Florida. Currently an undergraduate junior at the University of Florida, he is pursuing a B. S. in Biomedical Engineering with minors in Mathematics and Computer Science. He is interested in attending medical school and engaging in surgery.

Mike Fortunato earned his B. S. and M.S. in Materials Science and Engineering at the Pennsylvania State University in 2012 and 2015. He joined the Colina Research Group at the University of Florida in 2015 and is a Ph.D. candidate. 


\section{PRESS SUMMARY}

Research indicates that disulfide bonds may play an important role in the stabilization of protein structure and conformation. In this study, atomistic molecular dynamics simulations were performed on Immunoglobulin G and Bovine Serum Albumin. A previously established prescreening methodology was extended to both proteins to identify disulfide bonds with significant contributions to local structure, supporting the reproducibility of the methodology. The prescreening methodology can therefore be applied as a predictive tool to gauge the relative importance of disulfide bonds to structural stability, which can then be experimentally tested. This work provides insight into the role of these specific disulfide bonds in the stabilization of protein structure. 


\title{
Syndecan Affects Odor Response as well as Learning and Memory in Drosophila melanogaster
}

\author{
Dena Arizanovska*, Jonathan King*, \& Karl G. Johnson \\ Department of Neuroscience, Pomona College, Claremont, CA \\ Students:da032014@pomona.edu*,jbk02014@pomona.edu \\ Mentor:karl.johnson@pomona.edu
}

\begin{abstract}
Syndecan $(\mathrm{Sdc})$ is a transmembrane heparan sulfate proteoglycan that plays a crucial role in axon guidance and synapse formation during CNS development in Drosophila melanogaster. To further examine the effect of syndecan on CNS function, Sdc ${ }^{23}$ mutant $D$. melanogaster larvae were used to examine odor preference and the capacity for learning and memory. A series of olfaction assays in both wild type and mutant larvae were performed to characterize naive odor responses before adding a training period to identify the capacity for associative learning. These results showed that $\mathrm{Sdc}^{23}$ larvae prefer odors that wild type larvae do not respond to, suggesting a difference in odor receptor pathways and wiring. In addition, associative learning has been documented in wild type larvae, yet no evidence of associative learning in $\mathrm{Sdc}^{23}$ larvae was found, suggesting that the syndecan also plays a role in learning and memory in D. melanogaster larvae.
\end{abstract}

\section{KEYWORDS}

Syndecan; Proteoglycans; Neurodevelopment; Axon Guidance; Olfaction; Attraction Index; Associative Learning; Drosophila

\section{INTRODUCTION}

Olfactory systems are critical in most organisms because chemical cues in the environment provide essential information about a wide range of stimuli such as food, predators, mates, and pathogens. ${ }^{1}$ Many of these interactions are made possible because no physiological interaction is needed between organisms and these cues. This allows organisms to make behavioral decisions at longer distances from their cues. ${ }^{2}$ Because olfaction is so important for organism survival, the development and function of the olfactory system is very highly regulated. In Drosophila melanogaster, the olfactory system gets organized twice; first, during development from the egg to larval stage, and then again when the larvae enters the pupal stage before becoming an adult. ${ }^{3}$ Because this system is so well organized, it is a valuable area to study in order to better understand the distinct roles of genes, proteins, and compounds, such as proteoglycans, as they relate to basic neural processes such as axon guidance.

Insects provide a great platform through which to study olfaction because of the shared qualities their olfactory systems have with mammals. Most notably, both mammalian and insect olfactory systems operate under the principle that every glomerulus, or cluster of nerve endings in the olfactory bulb, receives input from one class of olfactory receptor neurons and each individual olfactory receptor neuron expresses a single odorant receptor. ${ }^{4}$ At the level of the receptor, odorants primarily interact with transmembrane proteins through a signaling process in the cilia, which extend from olfactory receptor neurons. ${ }^{2}$ Although the sizes of insect and vertebrate olfactory systems are highly variable, these core similarities are very well conserved. Maintaining so much similarity in their organization of the olfactory system with vertebrates, while also having a relatively simple nervous system, is what makes $D$. melanogaster an excellent model organism. ${ }^{5}$ Furthermore, the different olfactory receptors and olfactory receptor genes expressed in $D$. melanogaster are very well characterized in both the larval and adult stages, making it an ideal model organism for studying olfaction as it relates to neurodevelopment. ${ }^{3}$

Proteoglycans are a particular topic of interest in neurodevelopment because of their crucial roles in neurogenesis, axon guidance, and synaptogenesis in the central nervous system. ${ }^{6-7}$ They are expressed in the extracellular matrix (ECM) of many tissues, including the vertebrate spinal cord and the brain, where they have been implicated in controlling neural development by activating various growth factors. ${ }^{8}$ Heparan sulfate proteoglycans (HSPGs) are abundant cell surface molecules in the ECM that have extremely important functions for ECM-mediated neurodevelopment, specifically axon guidance. ${ }^{9}$ The structure of HSPGs consists of a core protein that is covalently bound to the highly charged glycosaminoglycan (GAG) side chain heparan sulfate (HS), a close structural relative of heparin. ${ }^{10}$ These HS side chains are disaccharides that can exist on their own, but usually covalently attach to the HSPG core at a serine residue when they're found in vivo. ${ }^{11}$

While the functions of HSPGs are very diverse, one of their most important roles is in guiding developing axons along their proper neuronal pathways during development. ${ }^{6}$ Studies in both vertebrates and invertebrates have shown that mutations in 
HSPG core proteins lead to a variety of phenotypes with different neuronal connectivity, implying that they have a significant contribution in axon path guidance and synapse formation during development. ${ }^{11}$ This study focuses on a specific type of HSPG called syndecan (Sdc) that localizes to developing axons and is necessary for axon growth cone navigation in the CNS. ${ }^{12}$ Syndecan core proteins are highly evolutionarily conserved type 1 transmembrane proteins with multiple HS side chains. Syndecan is expressed at the cell surface in the nervous system of mammals and has homologs in a diverse array of species, including Drosophila melanogaster, Caenorhabditis elegans, and Xenopus laevis. ${ }^{13-14}$

In Drosophila, syndecan is a necessary component of Slit/Robo signaling pathway at the Drosophila central nervous system midline, and the axons of syndecan mutant embryos have frequent midline crossing defects. ${ }^{12,15}$ Syndecan has been shown to facilitate the interactions between Slit and Robo, ${ }^{16}$ allowing Slit and Robo signaling to regulate axon guidance in the CNS, including odorant receptor neuronal projections to glomerular targets. ${ }^{17}$ Because Slit and Robo play crucial roles in regulating the development of the olfactory system in Drosophila, we hypothesized that Syndecan might also influence olfactory system development using a similar mechanism to how Sdc works in the developing embryonic central nervous system. To explore this on a behavioral level, we hypothesized that Sdc mutant Drosophila larvae will be attracted to different odorants than wild-type larvae due to a lack of proper olfactory wiring.

In addition, it is possible that Syndecan plays a role in other functions of the CNS, such as learning and memory, that have not yet been fully explored. Currently, there is some evidence that as a member of the ECM, HS signaling is important for learning and memory; HSPG knockout mice have been found to display an array of behavioral problems. Furthermore, mice that have been treated with heparinase (a substance that removes HSPGs locally) have demonstrated decreased LTP and hippocampal formation of contextual memories. ${ }^{18}$ Therefore, it would be useful to study the effects of an HSPG mutation on learning and memory in a much simpler model organism. Drosophila possess the cellular machinery for learning and memory as early as the first instar larval stage, and can be trained to avoid an odor that is presented in tandem with a shock. ${ }^{19}$ Drosophila larvae are also capable of associative learning and memory using either attractive (fructose) or aversive (quinine) gustatory cues to induce changes in naive odor preference. ${ }^{5}$ However, the role of syndecan in Drosophila learning and memory has not yet been characterized. This study therefore aims to address the hypothesis that syndecan is necessary for learning and memory by assessing changes in third instar $\mathrm{Sdc}^{23}$ Drosophila melanogaster larvae odor preference following an associative conditioning training protocol. Given the previous literature showing that HSPGs regulate learning and memory in vertebrate systems, we hypothesize that Sdc mutants larvae will also show impaired associative learning and memory compared to control larvae for odors that are paired with gustatory cues.

\section{METHODS AND PROCEDURES}

Drosophila Stocks

Drosophila were reared in bottles of standard fly food at $25^{\circ} \mathrm{C}$, and adult flies were transferred to new bottles every two days. $\mathrm{Sdc}^{23}$ loss of function mutants have been previously described as a deletion that removes the start codon and the first two exons of Sdc. ${ }^{20} \mathrm{Sdc}^{23}$ mutants were balanced over CyoGFP and reared in bottles of instant fly food (Formula 4-24; Carolina Biological Supply Company) on a 12:12 light dark cycle at $25^{\circ} \mathrm{C}$. Homozygous $\mathrm{Sdc}^{23}$ mutants were selected on a fluorescent dissecting microscope by selecting against GFP, and were transferred to odorant testing chambers.

Larval Chemotaxis Assays

Olfaction assays were performed for ten different odorants: ethyl acetate, isoamyl acetate, pentanol, hexanol, hexan-3-ol, heptanol, hexanal, hex-2-enal, toluene, and ethyl proprionate. Pure odorants ( $2 \mu \mathrm{L}$; Sigma Aldrich) were pipetted onto a small piece of Kimwipe in an Eppendorf tube cap on one side of a petri dish on a layer of $1.5 \%$ agar. Water $(2 \mu \mathrm{L})$ was pipetted onto a small piece of Kimwipe in an Eppendorf cap on the opposite side of the petri dish to serve as a control. Fifteen larvae per trial were placed in a straight line down the center of the Petri dish, and allowed to chemotax freely for two minutes. Larval position was marked at the end of the assay, and an "Attraction Index" was calculated by dividing distance from the odorant by the sum of their distance from the odorant and water $(\mathrm{a} /(\mathrm{a}+\mathrm{b})$. Attraction Index values close to 0 indicated that larvae crawled toward the odor, while Attractive Index values close to 1 indicated that larvae crawled away from the odor (Figure 1). This protocol was repeated 6 times for a total of 90 wild-type and $90 \mathrm{Sdc}^{23}$ larvae per odor assay. 

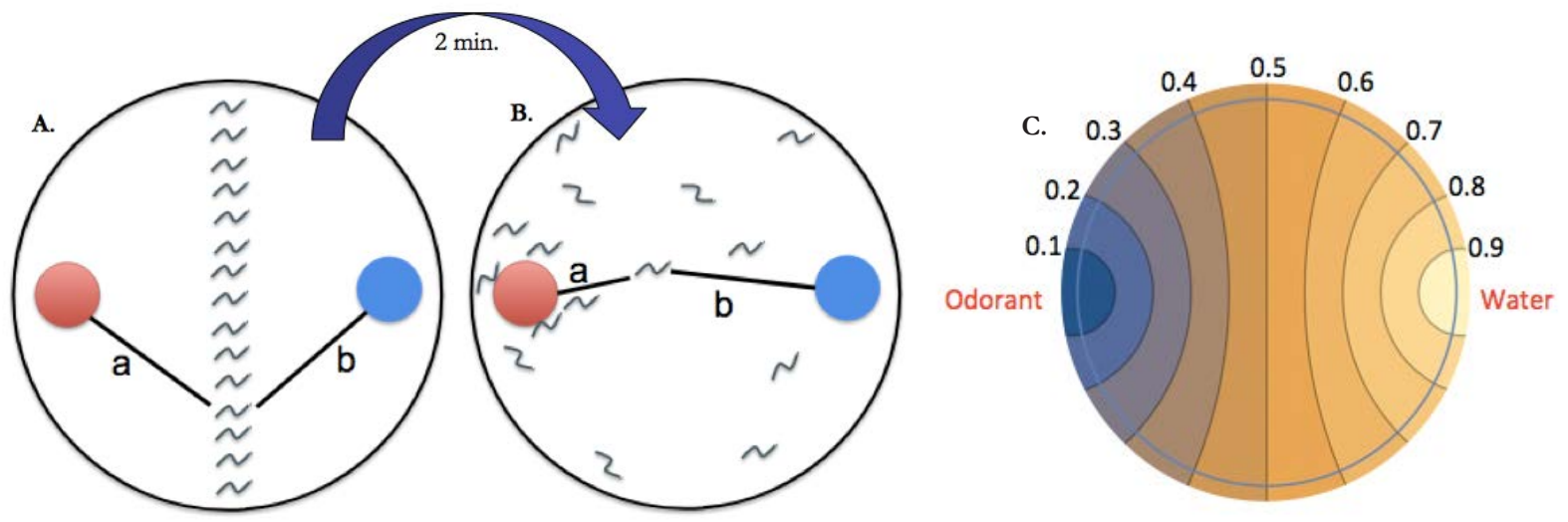

Figure 1. Olfaction assay testing naïve odor versus water preference. A) Olfaction assay setup at the beginning of a trial where fifteen larvae are placed in the center of a Petri dish, equidistant from an odorant on one side and water on the other. B) Olfaction assay following the 2 minute chemotaxis period, where $a$ is the final distance of each larvae from the odorant and $b$ is the final distance from the water. $\mathrm{C})$ Schematic representation of $\mathrm{a} /(\mathrm{a}+\mathrm{b})$ values for larval positions following chemotaxis.

Some of the odorants identified as significantly attractive were then tested against each other to assess larval preference for one odorant over another using the same protocol as before. To measure wild-type and $\mathrm{Sdc}^{23}$ mutant ability for learning and memory, larvae underwent an associative learning training protocol. ${ }^{21}$ Fifteen larvae were placed in the center of a "Training Plate" prepared with $2 \mathrm{M}$ fructose dissolved in the $1.5 \%$ agar with odorant $(2 \mu \mathrm{L})$ in Eppendorf tube caps on either side of the dish. After one minute of chemotaxis, larvae were transferred to a second petri dish that was prepared without fructose and contained a different odorant $(2 \mu \mathrm{L})$ for one minute. Larvae were then transferred to a petri dish without odorant or fructose for one minute of rest. This protocol was repeated four times before larvae were transferred to a chemotaxis assay as described above, with the odorant they were pre-exposed to with fructose against the other. The training was done reciprocally by switching which odorant was paired with fructose to control for differences due to odorant preference. Odorants tested against each other were hexanol versus ethyl acetate, hexanol versus pentanol, and heptanol versus ethyl proprionate, and a total of 90 wild-type and $90 \mathrm{Sdc}^{23}$ mutant larvae were tested per each condition.

\section{Quantification of Results}

Larval position was marked on the Petri dish cap using a Sharpie marker immediately following the two-minute chemotaxis period. Larval position was used to calculate the Attraction Index for each odorant as described above. A two-tailed independent samples t-test of the average attraction index for each odorant vs. water condition was conducted against a water vs. water control in Excel. A two-tailed independent samples t-test of the average attraction index after training with fructose against the corresponding naïve odor versus odor attraction index was conducted in Excel. All statistical analyses were subject to Bonferroni corrections for multiple samples.

\section{RESULTS}

Naïe Olfaction Assays

In order to determine if $\mathrm{Sdc}^{23}$ mutant larvae have different odor preferences from control larvae, olfaction assays were performed to examine 90 wild-type and $90 \mathrm{Sdc}^{23}$ mutant larvae odorant preferences. A two-tailed independent samples t-test comparing responses of larvae to odorant versus water against control water versus water assays indicated that wild-type and $\mathrm{Sdc}^{23}$ larvae are attracted to different odorants. Naïve odorant versus water assays indicated that wild-type larvae are significantly attracted to ethyl acetate, pentanol, hexanol, hexan-3-ol, and hex-2-enal (Figure 2). Sdc ${ }^{23}$ mutant larvae demonstrate significant attraction to hexanol, hexan-3-ol, and ethyl proprionate (Figure 2). Two-tailed independent samples t-tests indicated that wild-type and Sdc ${ }^{23}$ larvae exhibit significantly different responses to ethyl acetate, pentanol, hexanol and hex-2-enal (Table 1). 


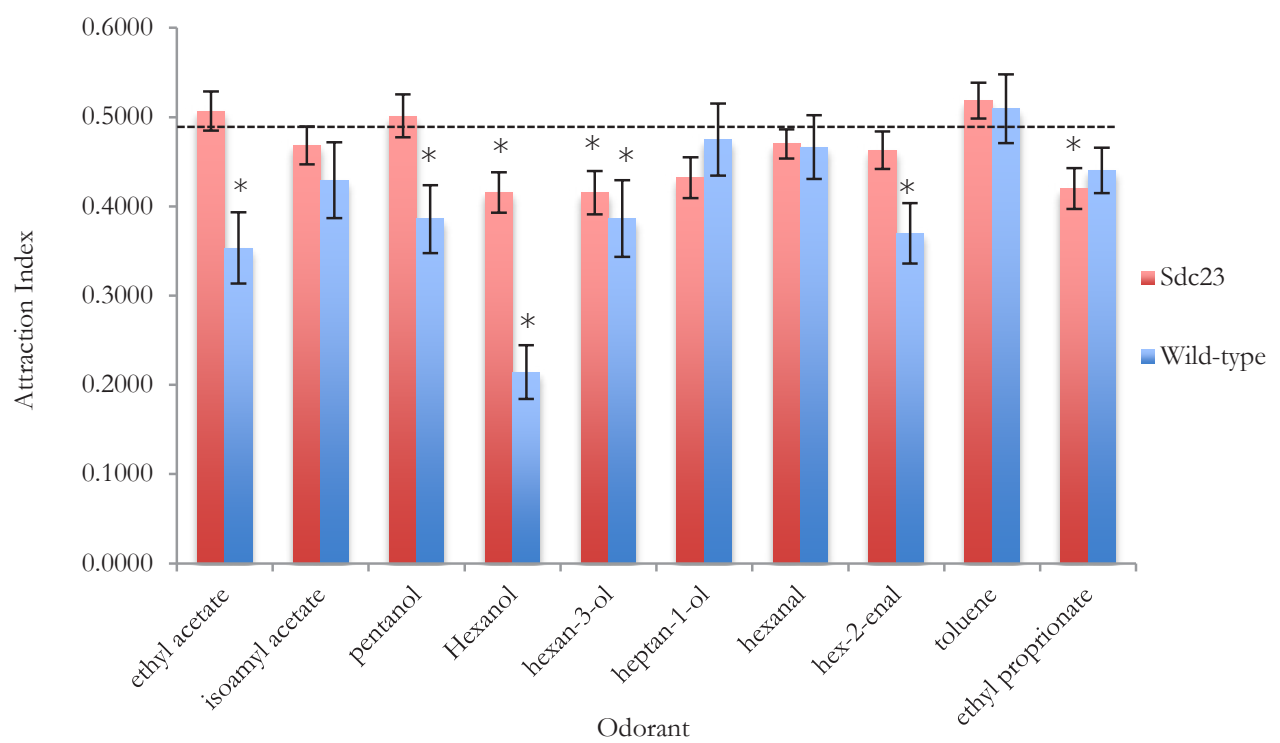

Figure 2. Wild-type and $\mathrm{Sdc}^{23}$ larvae show attraction to different odorants. Attraction index for wild-type and $\mathrm{Sdc}^{23}$ larvae in response to different odorants. Error bars denote the standard error of the mean and stars indicate attraction at the .005 significance level (significance was established by comparing responses to the water vs. water control with Bonferroni correction for multiple comparisons). The dashed line represents a water vs. water control condition with an average attraction index of $.48(\mathrm{SEM}=.03, \mathrm{n}=265)$. Note: Attraction index values less than 0.5 indicate increased attraction to an odorant over the water control.

\begin{tabular}{|c|c|c|c|c|c|c|c|}
\hline & $\begin{array}{l}\text { Ethyl } \\
\text { Acetate }\end{array}$ & Pentanol & Hexanol & Hexan-3-ol & Heptanol & Hex-2-enal & $\begin{array}{l}\text { Ethyl } \\
\text { Proprionate }\end{array}$ \\
\hline Wildtype & $\nu$ & $\nu$ & $\nu$ & $\nu$ & & $\nu$ & \\
\hline $\mathrm{Sdc}^{23}$ & & & $\nu$ & $\nu$ & & & $\nu$ \\
\hline $\begin{array}{l}\text { Significant } \\
\text { Difference }\end{array}$ & Yes & Yes & Yes & No & No & Yes & No \\
\hline
\end{tabular}

Table 1. Attractive Odors for Wild-type and Sdc ${ }^{23}$ Mutant Larvae. Check marks indicate the odorant was significantly attractive. The third row indicates whether or not the attraction index for each odor was significantly different between wildtype and Sdc ${ }^{23}$ mutant larvae at the .007 significance level (significance was established by comparing behavioral responses between wildtype and $\mathrm{Sdc}^{23}$ larvae with Bonferroni correction for multiple comparisons).

To determine naïve odor preference measures, wildtype and mutant larvae were put in an odor choice assay with two different odors to choose from. When given a choice between hexanol and ethyl acetate, wildtype larvae exhibit a slight preference for hexanol over ethyl acetate. Following an associative learning protocol where hexanol was paired with the gustatory reward fructose, wildtype larvae exhibited a significant increase in their preference for hexanol $(\mathrm{p}<.01)$. Sdc ${ }^{23}$ larvae also display a slight naïve preference for hexanol over ethyl acetate, however, they displayed no increase in preference for hexanol following the same training protocol (Figure 3). 


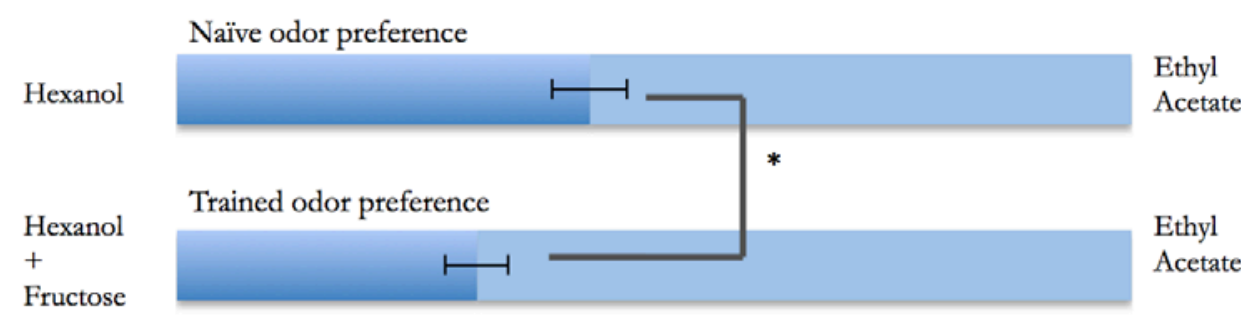

Naïve odor preference

Hexanol

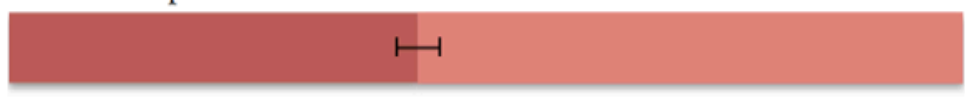

Ethyl

Acetate

Trained odor preference

Hexanol

$+$

Fructose

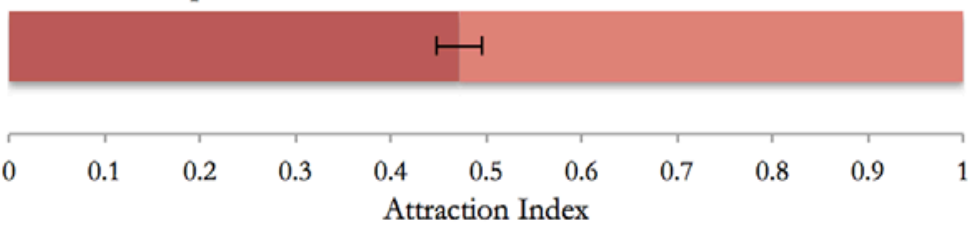

Ethyl

Acetate

\section{Wildtype}

Sdc23

Figure 3. Wild-type but not $\mathbf{s d c}^{23}$ larvae learn to associate hexanol with fructose reward. Comparison between naïve odor preference behavior and odor preferences following fructose training. Error bars indicate the SEM, and stars indicate evidence of successful learning at the .05 significance level ( $\mathrm{p}=.0022$ for wild-type larvae, $\mathrm{p}=0.19$ for $\mathrm{sdc}^{23}$ larvae).

Conversely, when ethyl acetate was paired with the gustatory reward fructose during the training protocol, wildtype larvae exhibited a significant shift in preference for ethyl acetate over hexanol. Again, $\mathrm{Sdc}^{23}$ larvae exhibited no behavioral changes in response to this training (Figure 4).

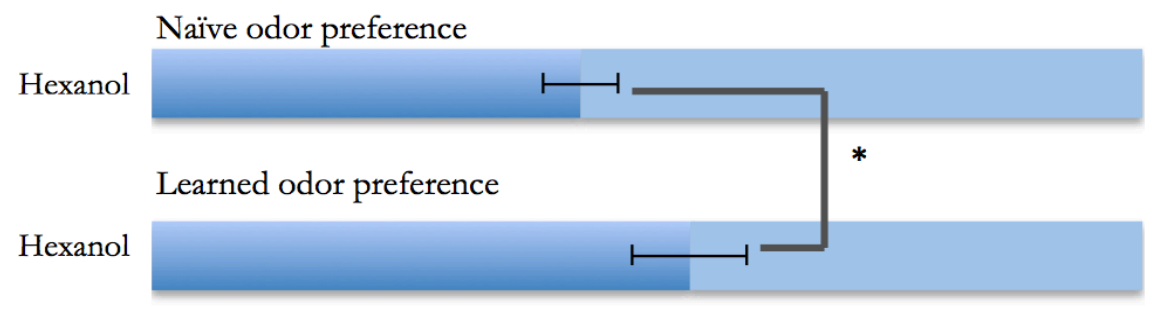

Ethyl

Acetate

Naïve odor preference

Hexanol

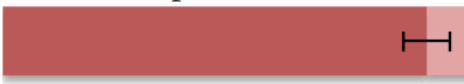

Wildtype

Ethyl

Acetate +

Fructose

Ethyl

Acetate

Sdc23

\section{Learned odor preference}

Hexanol

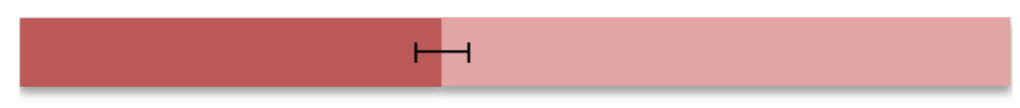

Ethyl

Acetate +

Fructose

\begin{tabular}{lllllllllll}
\hline & 0.1 & 0.2 & 0.3 & 0.4 & 0.5 & 0.6 & 0.7 & 0.8 & 0.9 & 1 \\
& \multicolumn{1}{c|}{ Attraction Index } & & & &
\end{tabular}

Figure 4. Wild-type but not $\mathbf{s d c}^{23}$ larvae learn to associate ethyl acetate with fructose reward. Comparison between naïve odor preference behavior and odor preferences following fructose training. Error bars indicate the SEM, and stars indicate evidence of successful learning at the .05 significance level ( $\mathrm{p}=.0064$ for wild-type larvae, $\mathrm{p}=.95$ for $\mathrm{sdc}^{23}$ larvae).

The inability for $\mathrm{Sdc}^{23}$ larvae to change their behavior in response to training could be due either to their inability to learn, or to their inability to smell the odorants. However, we knew that both wildtype and $\mathrm{Sdc}^{23}$ mutant larvae could smell hexanol, since it was significantly attractive to both sets of larvae. To further examine the ability to train wildtype and Sdc ${ }^{23}$ larvae, we conducted the same training protocol using another odorant previously demonstrated to be attractive to Sdc ${ }^{23}$ larvae; ethyl proprionate. Both wildtype and $\mathrm{Sdc}^{23}$ naïve larvae showed no significant preference for either odor. Following training where heptanol was paired 
with fructose, there was no significant change in preference for either wildtype or Sdc ${ }^{23}$ larvae (Figure 5). Similarly, no significant change in preference was observed for either genotype when ethyl proprionate was paired with fructose during training (Figure 6).
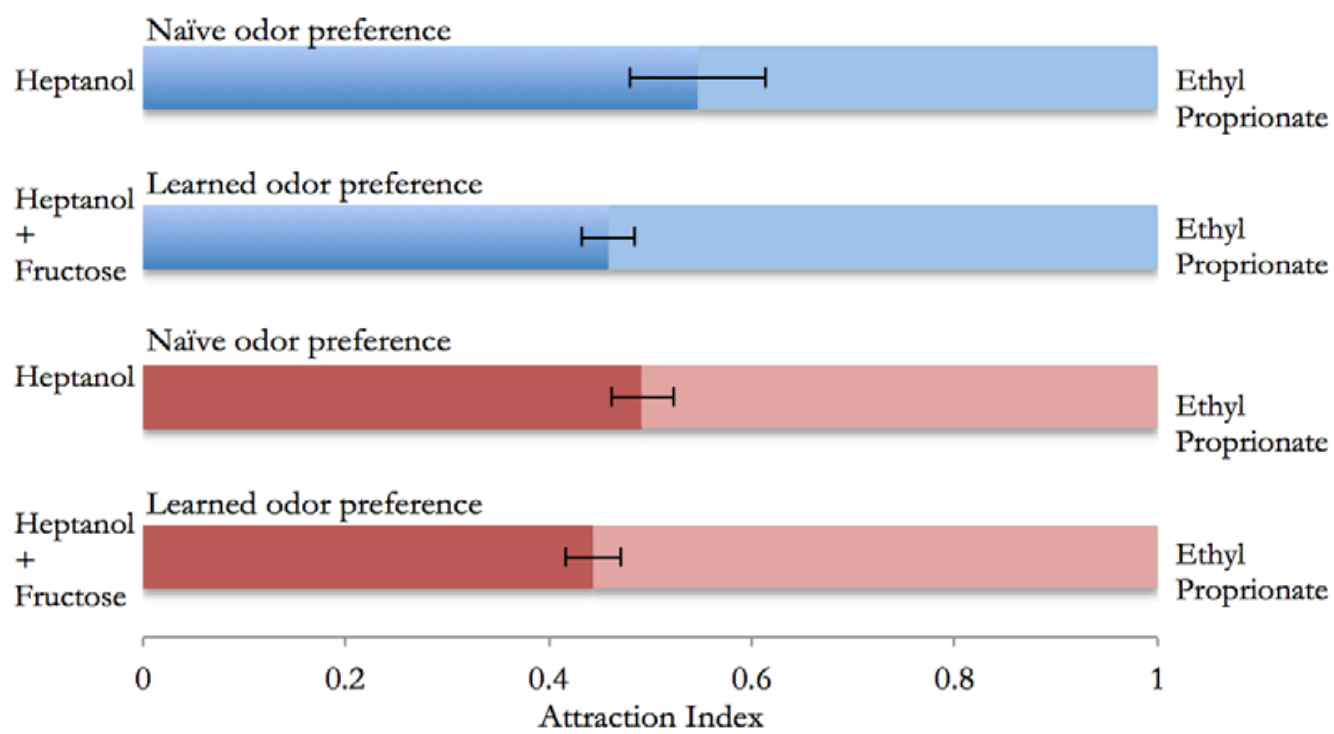

Figure 5. Wild-type and $\mathbf{s d c}^{23}$ larvae do not learn to associate heptanol with fructose reward. Comparison between naïve and learned odor preferences. Error bars indicate the SEM. There is no evidence of successful learning at the .05 significance level ( $\mathrm{p}=.13$ for wild-type larvae, $\mathrm{p}=.24$ for sdc ${ }^{23}$ larvae).

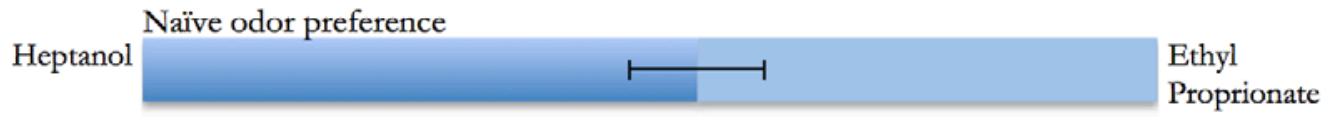

\section{Learned odor preference}

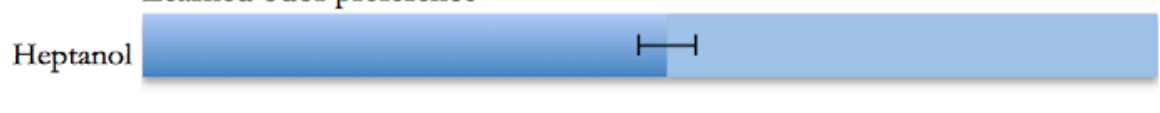

Ethyl

Proprionate

+ Fructose

Naïve odor preference

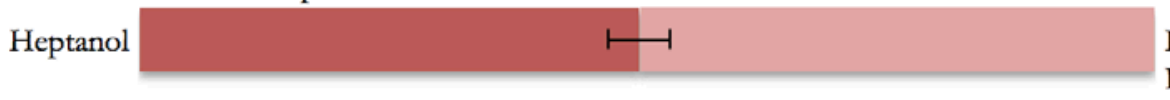

Ethyl

Proprionate 


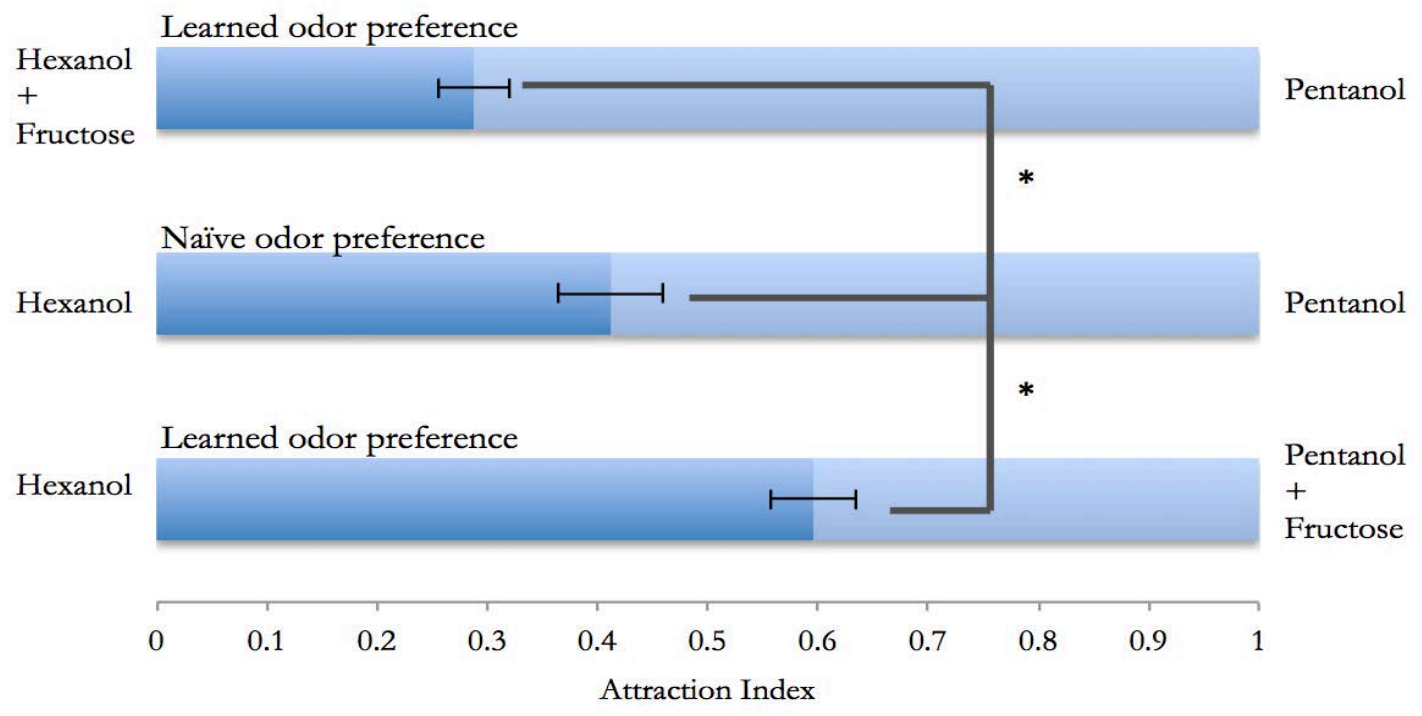

Figure 7. Wild-type larvae learn to associate hexanol and pentanol with fructose reward. Comparison between naïve and learned odor preferences. Error bars indicate the SEM, and stars indicate evidence of successful learning at the .05 significance level $(\mathrm{p}=.0027$ when hexanal is paired with fructose, $\mathrm{p}=.00065$ when pentanol is paired with fructose).

\section{DISCUSSION}

Wild-type larvae and $\mathrm{Sdc}^{23}$ larvae exhibit different preferences for different odors, as wild-type larvae are significantly attracted to ethyl acetate, pentanol, hexanol, hexan-3-ol, and hex-2-enal while Sdc ${ }^{23}$ larvae are significantly attracted to hexanol, hexan-3-ol, and ethyl proprionate. In addition, $\mathrm{Sdc}^{23}$ larvae have a weaker behavioral response to all odors they find attractive, suggesting a mutation in the syndecan gene may result in altered wiring of the larval olfactory systems. One possible mechanism for such a wiring defect is that syndecan is known to be a necessary axon guidance receptor and its absence leads to improper Slit/Robo signalling. ${ }^{12,15}$ Perhaps the absence of Sdc disrupts odorant receptor neuron projections to glomerular targets during neural development, or perhaps Sdc functions further downstream in the olfactory pathways. However, despite having less extreme attraction indexes than wild-type, $\mathrm{Sdc}^{23}$ larvae still exhibit a significant preference for several odorants over water, indicating that $\mathrm{Sdc}^{23}$ larvae are not simply anosmic.

Despite the fact that our associative learning training protocol was effective in training wild-type larvae, learning and memory was not exhibited by $\mathrm{Sdc}^{23}$ mutants. Olfaction assays performed following the associative learning training protocol showed $\mathrm{Sdc}^{23}$ larvae exhibit no change in odor preference after pre-exposure to odorants paired with the gustatory reward fructose. Conversely, wild-type larvae's attraction to either hexanol or ethyl acetate significantly increased after respective pairing with fructose, indicating that the absence of syndecan prevented mutant larvae from being able to either learn or recall a reward associated with an odor. The training protocol was repeated with heptanol and ethyl proprionate, once again, no evidence of learning and memory was observed for $\mathrm{Sdc}^{23}$ larvae, however this time wild-type larvae also failed to respond to training. This is likely due to the fact that wild-type larvae are not sensitive to either odorant, as neither heptanol nor ethyl proprionate on its own produced a significant attraction index in wildtype larvae. To confirm the validity of this protocol for assessing learning and memory, wildtype larvae underwent a third training procedure with hexanol and pentanol, two attractive odorants. Significant increases in preference after training was observed in wildtype larvae, supporting the hypothesis that the training protocol was robust, and that syndecan mutants have deficits in learning and memory in Drosophila melanogaster.

Since the absence of syndecan changes larval olfaction-mediated behaviors significantly, it is possible that other complex cognitive processes are affected as well. Not surprisingly, previous research has shown impairments to vision in Sdc mutant adult Drosopbila ${ }^{22}$, raising the possibility that $\mathrm{Sdc}^{23}$ mutants also have defects in gustatory system function as well. In this case, $\mathrm{Sdc}^{23}$ larvae would have been unable to detect the fructose in the associative training protocol, meaning their failure to respond to reward is due to gustatory rather than learning and memory deficits. Future studies could examine $\mathrm{Sdc}^{23}$ mutants for associative learning capabilities using tactile aversive cues as opposed to attractive or repulsive gustatory cues. Previous work demonstrates that wild-type Drosopbila are capable of learning to avoid odors that are paired with an electric shock. ${ }^{23}$ In such an experiment, if $\mathrm{Sdc}^{23}$ mutants do demonstrate learned aversion, then it would follow that syndecan plays an important role in gustatory system function, and that the failure to respond positively to fructose-paired odorants is due to an inability to detect fructose rather than a failure of learning and memory. 
In order to further explore the mechanism of Sdc function in the olfactory system and in learning and memory, it would be useful to attempt to rescue the Sdc mutant phenotype by expressing a Sdc transgene in different spatial and temporal patterns in the developing larval brain. For example, if syndecan is only necessary during development to establish proper axonal connections, the syndecan mutant phenotype should be rescued with embryonic expression of a Sdc transgene, perhaps using a heat-shock promoter. Likewise, if syndecan is only involved in learning and memory, mushroom body expression of a Sdc transgene may rescue this mutant phenotype. Elucidating the spatial and temporal requirements of Sdc expression would provide insight into the molecular mechanisms of syndecan function in the developing olfactory system.

\section{CONCLUSIONS}

$\mathrm{Sdc}^{23}$ mutant Drosophila larvae find different food odors to be attractive compared to wild-type larvae, and have a less pronounced behavioral response to those odors. In addition, $\mathrm{Sdc}^{23}$ mutant larvae cannot complete an associative learning task using gustatory cues. Together, this evidence indicates that the Syndecan gene mutation affects the wiring of the larval olfaction system and may be involved in the development of other cognitive processes, such as learning and memory. This finding is significant as previous studies have not explored the effects of Syndecan on memory, and further research should replicate these findings using different associative cues to determine if the $\mathrm{Sdc}^{23}$ mutation impairs gustation in addition to olfaction or if it affects learning and memory directly.

\section{ACKNOWLEDGEMENTS}

The authors thank the Pomona College Summer Undergraduate Research Program and the Pomona College Neuroscience Department for funding this study.

\section{REFERENCES}

1. Kaupp, U. B. (2010) Olfactory signaling in vertebrates and insects: differences and commonalities, Nat Rev Neuro 11, 188200.

2. Field, L. M., Pickett, J. A., and Wadhams, L. J. (2000) Molecular studies in insect olfaction, Insect Mol Bio 9, 545-551.

3. Gerber, B., and Stocker, R. F. (2007) The Drosophila Larva as a Model for Studying Chemosensation and Chemosensory Learning: A Review. Chem Sens 32, 65-89.

4. Couto, A., Alenius, M., and Dickson, B. J. (2005) Molecular, Anatomical, and Functional Organization of the Drosophila, Olfactory System. Curr Biol 15, 1535-1547.

5. Scherer, S., Stocker, R. F., and Gerber, B. (2003) Olfactory Learning in Individually Assayed Drosophila Larvae, Learn \& Mem 10, 217-225.

6. Properzi, F., and Fawcett, J. W. (2004) Proteoglycans and Brain Repair, Physio 19, 33-38.

7. Yamaguchi, Y. (2001) Heparan sulfate proteoglycans in the nervous system: their diverse roles in neurogenesis, axon guidance, and synaptogenesis, Sem in Cell \& Dev Biol 12, 99-106.

8. Kresse, H., and Schönherr, E. (2001) Proteoglycans of the extracellular matrix and growth control, J Cell Physio 189, $266-274$.

9. Zou, Y., and Lyuksyutova, A. I. (2007) Morphogens as conserved axon guidance cues, Curr Op in Neurobiol 17, $22-28$.

10. Bernfield, M., Kokenyesi, R., Kato, M., Hinkes, M. T., Spring, J., Gallo, R. L., and Lose, E. J. (1992) Biology of the syndecans: a family of transmembrane heparan sulfate proteoglycans, Ann Rev of Cell Biol 8, 365-393.

11. Van Vactor, D., Wall, D. P., and Johnson, K. G. (2006) Heparan sulfate proteoglycans and the emergence of neuronal connectivity, Curr Op in Neurobiol 16, 40-51.

12. Johnson, K. G., Ghose, A., Epstein, E., Lincecum, J., O’Connor, M. B., and Van Vactor, D. (2004) Axonal Heparan Sulfate Proteoglycans Regulate the Distribution and Efficiency of the Repellent Slit during Midline Axon Guidance, Curr Biol 14, 499-504.

13. Bandtlow, C. E., and Zimmermann, D. R. (2000) Proteoglycans in the Developing Brain: New Conceptual Insights for Old Proteins, Physio Rev 80, 1267-1290.

14. Bernfield, M., Götte, M., Park, P. W., Reizes, O., Fitzgerald, M. L., Lincecum, J., and Zako, M. (1999) Functions of cell surface heparan sulfate proteoglycans, Ann Rev of Biochem 68, 729-777.

15. Steigemann, P., Molitor, A., Fellert, S., Jäckle, H., and Vorbrüggen, G. (2004) Heparan Sulfate Proteoglycan Syndecan Promotes Axonal and Myotube Guidance by Slit/Robo Signaling, Curr Biol 14, 225-230.

16. Rhiner, C., Gysi, S., Fröhli, E., Hengartner, M. O., and Hajnal, A. (2005) Syndecan regulates cell migration and axon guidance in C. elegans, Dev 132, 4621-4633.

17. Jefferis, G. S. X. E., Marin, E. C., Stocker, R. F., and Luo, L. (2001) Target neuron prespecification in the olfactory map of Drosophila, Nat 414, 204-208.

18. Senkov, O., Andjus, P., Radenovic, L., Soriano, E., and Dityatev, A. (2014) Neural ECM molecules in synaptic plasticity, learning, and memory, in Progress in Brain Research Vol. 214., 53-80. 
19. Aceves-Piña, E. O., Quinn, W. G., Smith, K. D., Steinberger, E., and Rodriguez-Rigau, L. J. (1979) Learning in Normal and Mutant Drosophila Larvae, Sci 206, 93-96.

20. Schulz, J. G., Ceulemans, H., Caussinus, E., Baietti, M. F., Affolter, M., Hassan, B. A., and David, G. (2011) Drosophila syndecan regulates tracheal cell migration by stabilizing Robo levels, EMBO Rep 12, 1039-1046.

21. Neuser, K., Husse, J., Stock, P., and Gerber, B. (2005) Appetitive olfactory learning in Drosophila larvae: effects of repetition, reward strength, age, gender, assay type and memory span, Anima behavior 69, 891-898.

22. Rawson, J. M., Dimitroff, B., Johnson, K. G., Rawson, J. M., Ge, X., Van Vactor, D., and Selleck, S. B. (2005) The heparan sulfate proteoglycans Dally-like and Syndecan have distinct functions in axon guidance and visual-system assembly in Drosophila, Curr biol 15, 833-838.

23. Pauls, D., Pfitzenmaier, J. E. R., Krebs-Wheaton, R., Selcho, M., Stocker, R. F., and Thum, A. S. (2010) Electric shockinduced associative olfactory learning in Drosophila larvae, Chem sens 35, 335-346.

\section{ABOUT STUDENT AUTHORS}

Dena Arizanovska and Jonathan King are neuroscience majors at Pomona College who graduated in May 2018. Dena plans on attending graduate school and having a career in research. Jonathan plans on pursuing an M.D.

\section{PRESS SUMMARY}

This study examines the effects of the Syndecan $\left(\mathrm{Sdc}^{23}\right)$ gene on the development of Drosophila larvae's sense of smell. It was found the larvae with a mutated Syndecan gene prefer different odors than larvae with an intact Syndecan gene, suggesting that the Syndecan gene is necessary for the proper development of the olfactory system. Furthermore, this study examined whether Syndecan is involved in learning and memory by exposing larvae to certain odors with fructose to see if this would enhance future attraction to the odorant. While wildtype larvae showed increased attraction to odorants following pairing with fructose, Sdc ${ }^{23}$ larvae did not, suggesting an impairment learning and memory. This implies that the Syndecan gene is important for developing learning and memory abilities, and that it should be further studied for its role in higher cognitive functions. 


\title{
Investigation of Flow Rate on Chemical Bath Deposition of Silver Films Inside Hollow Polymer Cylinders
}

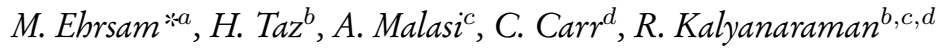 \\ ${ }^{a}$ Department of Mechanical, Aerospace and Biomedical, Engineering, University of Tennessee, Knoxville, TN. \\ ${ }^{b}$ Bredesen Center, University of Tennessee, Knoxville, TN. \\ ${ }^{c}$ Department of Chemical and Biomolecular Engineering, University of Tennessee, Knoxville, TN. \\ ${ }^{d}$ Department of Materials Science and Engineering, University of Tennessee, Knoxville, TN.
}

Students:mehrsam@vols.utk.edu*, htaz@vols.utk.edu, amalasi@vols.utk.edu, ccarr20@vols.utk.edu

Mentor:ramki@utk.edu

\begin{abstract}
In thin film solar cells made as planar structures, the absorption of solar light and hence, the solar conversion efficiency, is heavily determined by the position and orientation of the sun. One way to overcome this is to use solar cell geometries that could better absorb sunlight from different angles. One potential geometry is a hollow cylinder, which has the ability to better absorb the sun's rays incident at various angles due to the light trapping nature of the cylindrical geometry. If such solar cells could be fabricated inside polymer tubes with micrometer diameters, many potential applications built around non-woven or woven textiles could be realized. Here we investigate the deposition of homogeneous thin metallic films inside hollow polymer cylinders using the process of chemical bath deposition (CBD). Although films deposited via CBD have been studied extensively, mechanisms to achieve high quality deposition inside hollow cylinders are still not well understood. The objective of this project was to deposit a smooth, homogeneous silver film inside a hollow cylindrical structure using varying flow rates. The early stage film growth for very short deposition times was observed by the localized surface plasmon resonance of the silver nanoclusters via absorption spectra along the length of the tube. For longer deposition times, silver films formed and were analyzed for their morphology, thickness, roughness, and resistance using a combination of optical microscopy, scanning electron microscopy, and twoprobe conductivity. The findings from this study showed that deposition under flow with different Reynolds numbers had a strong influence on the morphology and electrical resistance of the deposited films.
\end{abstract}

\section{KEYWORDS}

Thin-films; Chemical Bath Deposition; Nanoparticles; Solar Cells; Silver

\section{INTRODUCTION}

Planar thin film semiconductors, which are the core of current generation solar cell technology, face fundamental challenges towards a continued reduction in cost while simultaneously increasing efficiency. For example, lower costs can be achieved by reducing the thickness of the semiconductor, eliminating materials, and reducing processing steps. However, ultrathin devices have significantly lower efficiency over bulk solar cell technology. For instance, Si ultrathin solar cell efficiencies are currently at $8-10 \%$, while that for bulk Si technology is at $15-25 \% .{ }^{1}$ The fundamental reason for this is the reduction in the amount of light trapped and absorbed in the ultrathin devices. A planar structure also has other disadvantages such as the efficiency and absorbency of the solar cell being heavily dependent on the position and orientation of the sun. Recently, a United States patent was awarded to a solar cell design in which the various metal and semiconductor layers of the solar cell were made by thin film deposition processes inside small diameter hollow cylinders, such as those made of polymer materials. ${ }^{2}$ The authors have suggested that the cylindrical geometry could better absorb the sun's rays due to its light trapping nature. In addition, the hollow nature of the substrate could also help in 


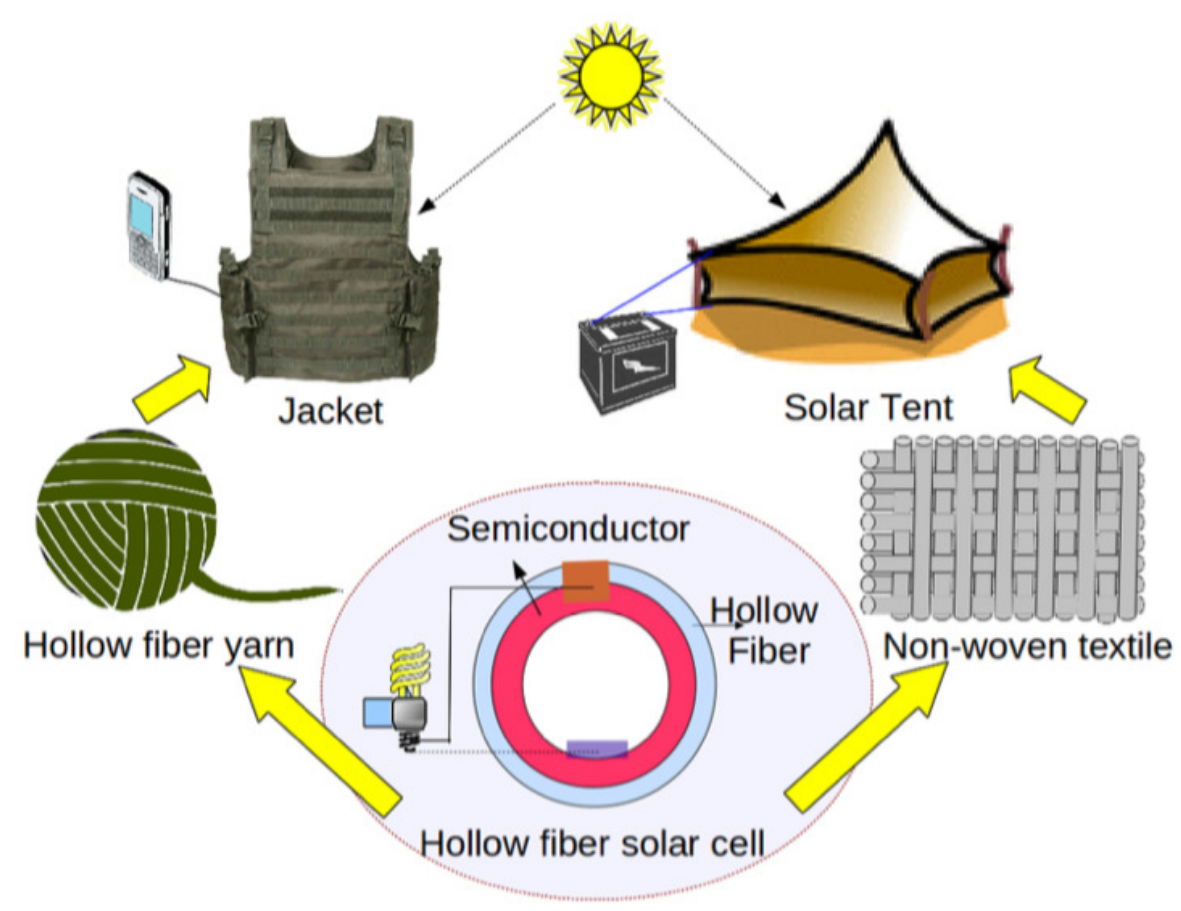

Figure 1. A schematic illustration of the potential applications that could be enabled by having a flexible hollow fiber solar cell. ${ }^{2}$

applications such as solar textiles as shown in Figure 1. Figure 1 depicts a semiconductor solar cell made inside a hollow polymer fiber. The vision is that this flexible hollow polymer fiber could be spun into a non-woven yarn or a woven fabric. Subsequently, the material can be transformed into a wearable device such as a jacket or a utilitarian device, such as a tent, from which solar energy can be harnessed.

A review of scientific literature has shown that there has been prior work on making organic and dye-sensitized solar cells on the outside of glass tubes or on fibers with a solid conducting core. ${ }^{3-7}$ Recently, the optical trapping properties of arrays of cylindrical radial junction solar cells motivated the fabrication of nanowire arrays of $\mathrm{Si}$ by various groups. ${ }^{8-12}$ However, there is little previous work on making inorganic solar cells inside hollow polymer fibers. Over the past decade, the chemical bath deposition (CBD) process has been successfully applied to deposit metal, semiconductor and dielectric films inside hollow fibers, largely for the purpose of infrared light transmission. ${ }^{13-25}$ In the CBD deposition process, salt solutions are mixed together at room temperature and flowed through the hollow fibers, resulting in a precipitation reaction of the desired metal, semiconductor or dielectric. ${ }^{26}$ The major focus of these previous works on deposition inside hollow fibers were on optimizing the film deposition conditions to obtain desired light transmission properties.

Here we present investigations of chemical bath deposition of silver ( $\mathrm{Ag}$ ) metal films by the CBD process inside $22 \mathrm{~cm}$ long and $0.8 \mathrm{~cm}$ diameter hollow polymer tubes. Depending upon the rate at which a fluid flows through the tube, the resulting velocity profile of the fluid may be stationary (i.e. flow velocity of zero), laminar (smooth flow) or turbulent, and this could greatly impact the deposition kinetics of the thin films. Furthermore, when the fluid enters the tube, the velocity profile typically requires a characteristic distance before achieving its steady-state behavior, potentially leading to differences in deposition at the entrance versus further inside the hollow tube. Therefore, the goal of this work was to understand how the morphology of silver thin films were affected by the rate of flow as well as with distance from the entrance of the fluid under the CBD process. We have utilized a combination of scanning electron microscopy (SEM) imaging, electrical resistivity measurements, and optical spectroscopy of the Ag plasmon properties to understand the $\mathrm{CBD}$ deposition of $\mathrm{Ag}$. 


\section{METHODS AND PROCEDURES}

For the $\mathrm{CBD}$ of $\mathrm{Ag}$ thin films, we utilized the known process in which two solutions were required. ${ }^{24}$ The first was a silver precursor made of a $8.3 \%$ molar Silver Nitrate (Acros Silver Nitrate 99.85\%) solution that was titrated using ammonium hydroxide to form $2 \mathrm{Ag}\left(\mathrm{NH}_{3}\right)_{2} \mathrm{OH}$. Next, a $25 \%$ molar sodium hydroxide [Fisher Scientific Sodium Hydroxide (beads)] solution was added to this silver solution, and was titrated again with ammonium hydroxide. The second solution was a reducing agent: a 6.7\% molar aqueous solution of dextrose [Fisher Scientific Dextrose (D-Glucose) Anhydrous]. The densities of these solutions are shown in Table 1. These solutions were then pumped through a Tygon Tube (ID: $1 / 16$ in) into a $22 \mathrm{~cm}$ long commercially available poly propylene tube (D\&W Fine Pack) with a $0.8 \mathrm{~cm}$ diameter.

\begin{tabular}{|c|c|}
\hline Solution & Density $\left(\mathrm{g} / \mathrm{cm}^{3}\right)$ \\
\hline \hline Silver Nitrate Solution & 1.014 \\
\hline Sodium Hydroxide Solution & 1.007 \\
\hline Reducing Agent Solution & 1.000 \\
\hline Final Solution & 1.007 \\
\hline
\end{tabular}

Table 1. Densities of Experimental Solutions

\section{Deposition at varying flow rates}

For the first part of this experiment, $200 \mathrm{~mL}$ of the final solution was allowed to flow at four different flow speeds, identified as low, medium, high, highest. From Table 1, it can be seen that the densities of these solutions were very similar to that of water. Therefore, the flow rates and velocities of the pump were approximated by timing $100 \mathrm{~mL}$ of water flow through the $1 / 16$ in $(.0016 \mathrm{~m})$ Tygon tubes. The Reynolds Number was then calculated using the Reynolds number equation for flow in a pipe shown in Equation 1:27

$$
R e=\left(\rho v_{a v g} D\right) / \mu
$$

Equation 1.

where the average velocity $\left(v_{\text {avg }}\right)$ of each of the speeds is shown in Table 2, the dynamic viscosity $(\mu)$ of water is $0.00089 \mathrm{~Pa}^{*}$, the density of water at room temperature $(\rho)$ is $1000 \mathrm{~kg} / \mathrm{m}^{3}$, and the diameter of the tube (D) is $\mathrm{D}=$ $0.0016 \mathrm{~m}$. Based on the calculation of the Reynolds number Re, presented in Table 2, all these speeds were in the laminar flow regime (i.e. $\operatorname{Re}<2600){ }^{27}$

\begin{tabular}{|c|c|c|c|c|}
\hline Pump Speed & Flow Rate $\left(\mathrm{m}^{3} / \mathbf{s}\right)$ & Velocity $(\mathrm{m} / \mathbf{s})$ & Reynolds Number $(\mathrm{Re})$ & Deposition time (s) \\
\hline \hline Low & $1.07 \times 10^{-7}$ & 0.053 & 95.4 & 1876 \\
\hline Medium & $1.91 \times 10^{-7}$ & 0.095 & 170.7 & 1048 \\
\hline High & $2.27 \times 10^{-7}$ & 0.113 & 203.3 & 880 \\
\hline Highest & $3.36 \times 10^{-7}$ & 0.167 & 300.2 & 596 \\
\hline
\end{tabular}

Table 2. Flow Parameters at Varying Speeds

The thickness and morphology of the deposited films were characterized at the entrance of the tube as well as at 10 and $20 \mathrm{~cm}$ distances along the tube using SEM (Zeiss Merlin). For this, the tubes were cut and samples were collected at the entrance of the tube and at the 10 and $20 \mathrm{~cm}$ positions. Prior to cutting the tubes, two-probe electrical conductivity (Keithley 2400) measurements were made for the total resistance of the deposited films along the entire length of the tube.

\section{Early stage deposition at highest speed}

For the second part of this experiment, the final solution was allowed to flow at the highest speed of $0.167 \mathrm{~m} / \mathrm{s}$ for 1 , 1.5 , and 2 minutes. The localized surface plasmon resonance (LSPR) peaks of the deposited silver nanoparticles were analyzed at various locations along the length of the tube in $2 \mathrm{~cm}$ increments from 2 to $20 \mathrm{~cm}$, using optical spectroscopy (Ocean Optics UV-Vis Spectrometer). Additionally, the concentration of the silver nanoparticles at 1, 1.5 and 2 minutes were analyzed using SEM (Phenom ProX). 


\section{RESULTS AND DISCUSSION}

I. Deposition at Varying Flow Rates

a. Morphology: Figure 2 shows plane-view SEM micrographs depicting the contrast resulting from the deposited Ag films at the three different locations and for the various flow rates mentioned in Table 1. Based on these images, it was clear that the Ag film morphology appeared to depend both on position along the tube as well as the flow rate.

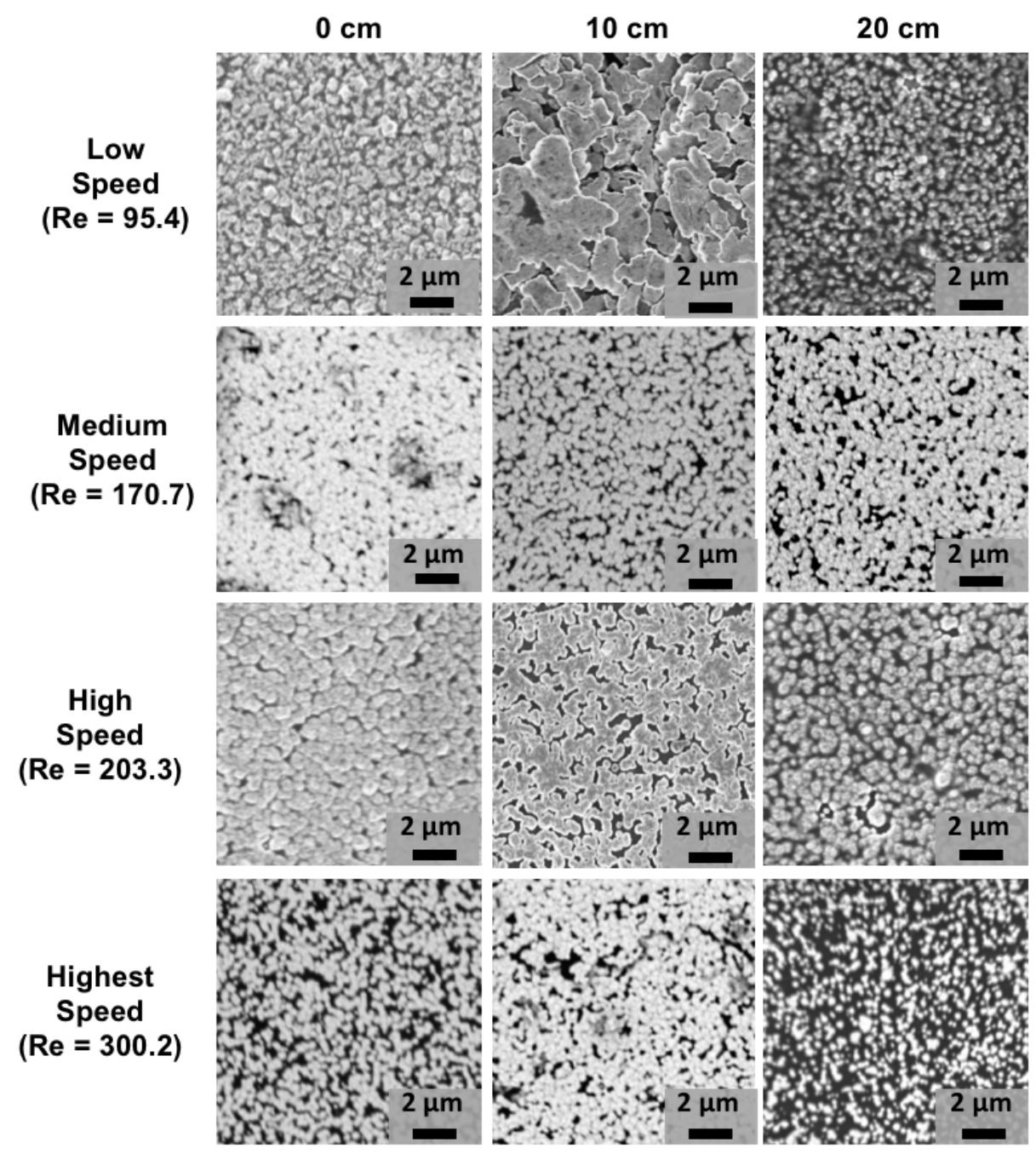

Figure 2. SEM images of deposited films at the Low, Medium, High, and Highest speeds (with the respective Re values indicated) at the entrance of the tube as well as 10 and $20 \mathrm{~cm}$ from the entrance.

For the low speed, there was a significant increase in the size of the $\mathrm{Ag}$ deposits in going from the entrance $(0 \mathrm{~cm})$ to $10 \mathrm{~cm}$, while the film appeared to consist of much finer features at $20 \mathrm{~cm}$. For the medium and high speeds, the film appeared to be fairly uniform along the length of the tube. It was also apparent that the microstructure became more aggregated or discontinuous towards the end of the tube. At the highest speed, the film appeared to have a smaller grained and discontinuous microstructure at the entry and end of the tube, with a more homogeneous deposit near the center (at $10 \mathrm{~cm})$.

b. Film thickness and deposition rate: After analyzing the plane-view morphology of the films, cross-sectional SEM was performed to analyze the thickness of the films for the four different speeds at the various locations (entrance $=0 \mathrm{~cm}$, middle $=10 \mathrm{~cm}$, near exit $=20 \mathrm{~cm}$ ). Figure 3(a) shows a typical cross-section of a Ag film deposited at low speed and 
taken at the entrance of the cylinder. The film thickness was estimated by averaging several thickness measurements (typically at least 5). Figure 3(b) plots the average thickness of the silver film at different locations of the tube as a function of the Reynolds number Re. The error bars for each measurement correspond to 1 standard deviation from the mean value.
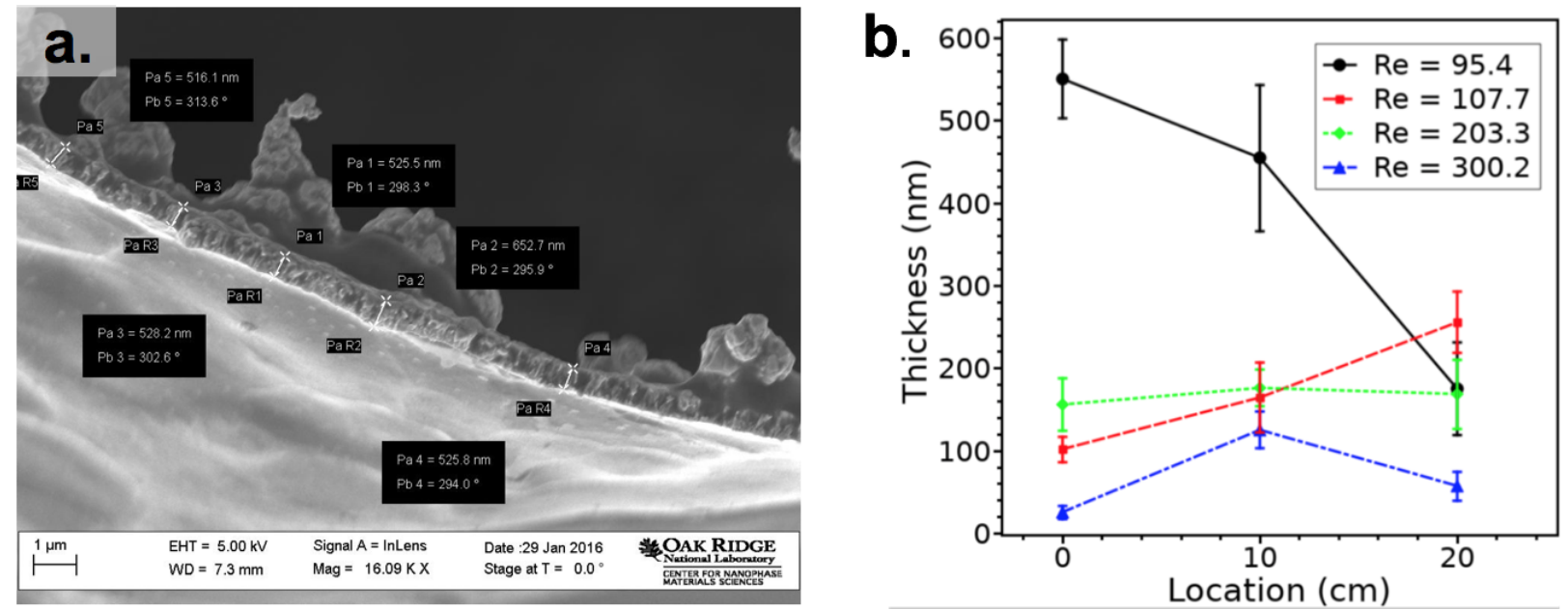

Figure 3. (a) Cross section SEM of silver film deposited at the low speed taken at the entrance of cylinder. (b) Thickness of film as a function of tube length.

In Figure 3(b), the thickness at each location was compared to the Reynolds number $R e$ for the various flow speeds. Near the entrance, the thickness dropped rapidly with increasing $R e$. In contrast to the low speed case with $R e=95$, the $R e=170$, showed an increase in film thickness across the length of the tube. The higher speed case of $R e=203$ showed a fairly uniform film thickness across the tube length. The highest speed (with $R e=300$ ) showed a much thinner film across the entire length as well as a larger thickness at the center of the tube $($ at $10 \mathrm{~cm})$ as compared to at the entrance and exit. For solar cell applications, it is important to have a homogeneous film thickness, and based on the thickness measurements in Figure 3(b), the high speed case with $R e=203$ appeared to be the most desirable for producing uniform thickness films. This suggested that for the conditions of our experiments, of tube diameter and flow rates, the $R e=203$ flow had the most optimal conditions of silver deposition along the inside walls of the tube.

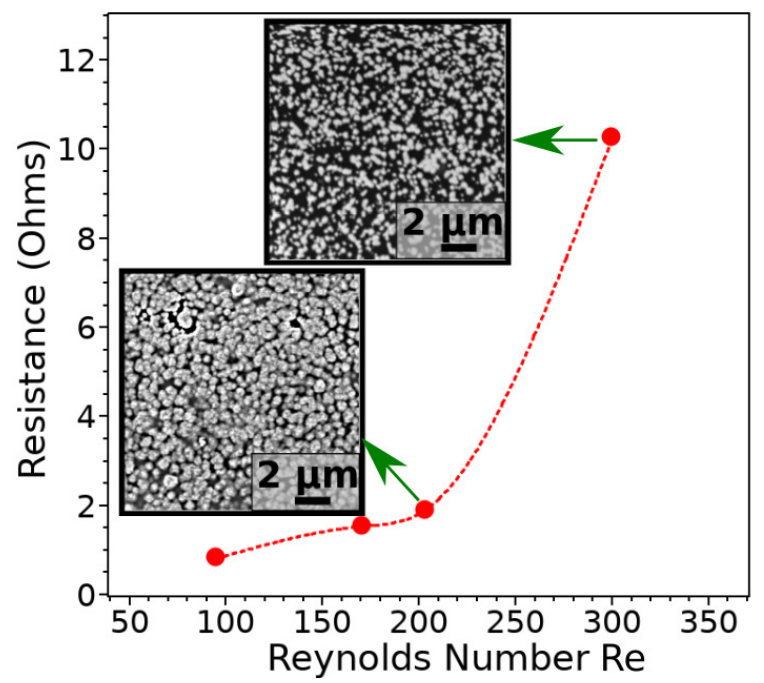

Figure 4. Measured resistance values of the deposited Ag films across the length of the hollow cylinder as a function of Re. SEM image of nanoparticles formed at $R e$ of 203 and 300 at the tube position of $10 \mathrm{~cm}$ are also shown. 
c. Film Resistance: In order to correlate the film thickness with film quality, the electrical resistance was measured by obtaining current versus voltage curves by placing electrical connections at either end of the tube so that the resulting behavior was representative of the entire length of the tube. From this data, the resistance versus Re (Reynolds number) was plotted and is shown in Figure 4. Based on these results, it is clear that for the films deposited with $95 \leq$ $R e \leq 203$, there was little change in overall film resistance. However, the highest speed with $R e=300$ resulted in nearly 5 fold increase in overall resistance across the tube. In order to understand why the resistance changed so significantly at the highest speed, the SEM images were analyzed. The insets in Figure 4 show the planar SEM images of the films at $\operatorname{Re}=203$ (high speed) and $\operatorname{Re}=300$ (highest speed). As mentioned earlier, with respect to Figure 3(b), the $R e=300$ case had the smallest thickness. Based on the SEM image shown in Figure 4, the microstructure for this case showed Ag deposit (bright regions) along with a large fraction of the dark regions, which corresponded to a lack of $\mathrm{Ag}$ deposit, suggesting poor connectivity between the Ag regions. In contrast, the SEM for the $R e=203$ case shown in Figure 4 indicated a much higher degree of connectivity between the bright regions corresponding to the Ag deposits. Therefore, the conclusion was that the higher resistance in the $R e=300$ case is a result of a poorly connected thin film morphology resulting from an overall thinner film.

\section{Early Stage Deposition at Highest Speed}

From the results of section I it became apparent that the Re was influencing the microstructure and overall continuity of the Ag films. In order to further understand this, we investigated the early stages of deposition as well as film growth as a function of time utilizing a combination of SEM (Phenom ProX) and optical spectroscopy.

\section{Minute}

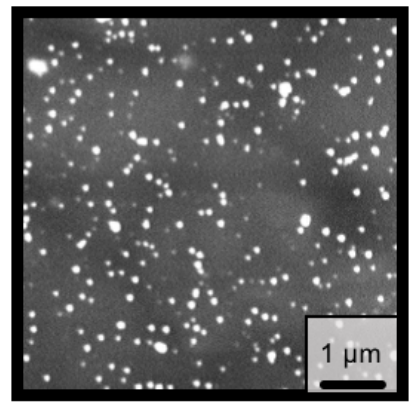

1.5 Minutes

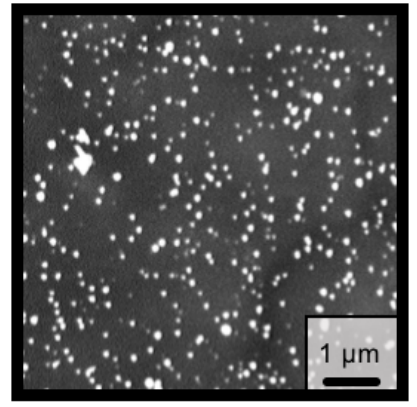

2 Minutes

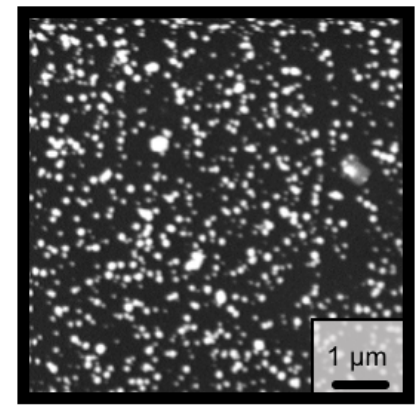

Figure 5. SEM images of Ag nanoparticles at $10 \mathrm{~cm}$ after 1, 1.5, and 2 minutes.

a. SEM investigation of Ag deposition with time: In Figure 5, SEM images taken following deposition for 1, 1.5, and 2 minutes of solution flowing at the highest $R e=300$ speed are shown. From the scale bar, it is evident that the Ag deposits at the earliest time are in the nanometer size scale. Further, increasing flow time leads to an increasing concentration of the number of these nanoparticles. At 3 minutes, evidence for agglomeration resulting in larger particles is also apparent. From this analysis, the high resistance of the $R e=300$ film measured in Figure 4(b) is supported by a deposition process that proceeds by deposition of discontinuous clusters of $\mathrm{Ag}$, thus making it harder to form a wellconnected Ag thin film microstructure.

b. Localized Surface Plasmon Resonance (LSPR) of Ag Nanoparticles (NPs): We also utilized optical spectroscopy to characterize the early stages of film deposition because Ag nanostructures show strong LSPR signal due to the conduction electrons. ${ }^{28}$ When light or electromagnetic waves interact with the Ag NPs, the electron cloud in the NPs oscillate collectively at a particular resonant frequency, giving rise to the LSPR peak. ${ }^{29}$ These resonances occur at specific wavelengths (or frequencies) that depend on particle size, shape, and the type of surrounding medium. ${ }^{30}$ Thus by observing the position of these resonances via optical spectroscopy, it is possible to determine information about the Ag nanos- 


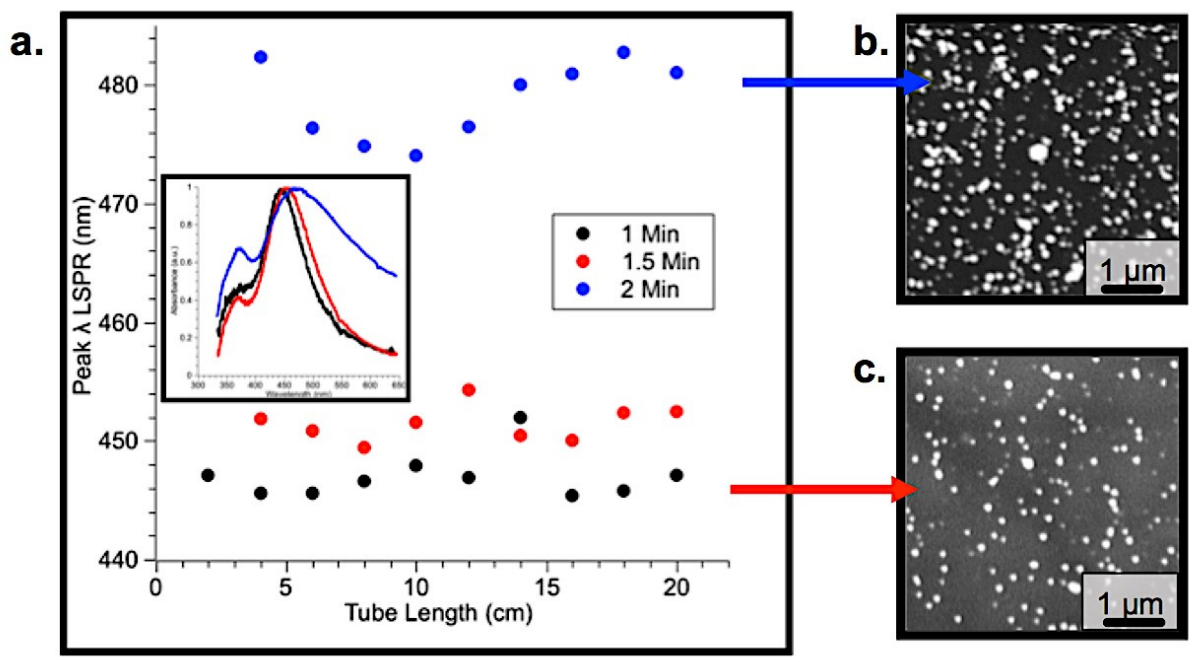

Figure 6. (a) Peak LSPR wavelengths as a function of tube length after 1, 1.5, and 2 minutes. (b) SEM image of nanoparticles after two minutes at $10 \mathrm{~cm}$ c. SEM image of nanoparticles after 1.5 minutes at $10 \mathrm{~cm}$.

tructures. In Figure 6(a) the wavelength of the LSPR peak measured at various locations along the length of the tube are plotted for the three early stage deposition times. The inset of Figure 6(a) shows typical absorbance curves from the middle of the tube for the three deposition times of 1, 1.5 and 2 mins. The LSPR wavelength corresponds to the intense peak visible around $450 \mathrm{~nm}$. From the results in Figure 6(a), the resonance wavelength appears to increase by a small amount in going from 1 to 1.5 minutes. From the SEM image in Figure 5, this increase is most likely due to a small increase in the average size of the Ag nanoparticles, which typically results in a red shift of the LSPR wavelength. More dramatic is the large red shift from the Ag deposited for 2 minutes, as seen by the blue dots in Figure 6(a). This large shift is most likely an indication of the substantial agglomeration happening in the Ag films, consistent with the SEM information shown in Figure 5. This result also suggests that LSPR could be used to monitor the deposition process in real-time in order to observe the transition from deposition of discrete islands of $\mathrm{Ag}$ to one where the agglomeration begins. Indeed, recently in-situ investigations have utilized Ag LSPR to monitor the deposition of thin films. ${ }^{31}$

\section{CONCLUSIONS}

In this paper, we explored the role of Reynolds number on the deposition of Ag thin film inside hollow cylindrical structure via the chemical bath deposition process. By utilizing a combination of scanning electron microscopy and electrical resistivity it was found that the uniformity of a film requires an optimal speed. More specifically, it was found that the Reynolds number of 203 provides a good balance between the deposition rate of the silver precipitates and the the formation of a film with fairly consistent film thickness throughout the length of a $22 \mathrm{~cm}$ hollow fiber with 0.8 $\mathrm{cm}$ inner diameter. The film quality was also verified by the overall low electrical resistance for this flow condition. Analysis of the early stage film deposition using SEM and localized surface plasmon resonance spectroscopy showed that the highest flow rate $(R e=300)$ produces segregated Ag film deposits at the early stage, likely resulting in the high resistivity of these films. Overall, these results show that controlling the flow rate can have substantial impact on the growth and ensuing morphology and physical properties of Ag thin films deposited inside hollow cylinders.

\section{ACKNOWLEDGMENTS}

This research was supported by NSF grant CBET-1349507 and TNSCORE grant NSF-EPS-10004083. This work was also supported by the Research and Instructional Strategies for Engineering Retention (RISER) at the University of Tennessee, Knoxville which is funded by the NSF through the Science, Technology, Engineering, and Mathematics Talent Expansion Program (STEP) award number 1068103. RK and HT acknowledge Oak Ridge National Laboratory CNMS grant CNMS2013-284 and University of Tennessee Department of Materials Science for SEM access. 


\section{REFERENCES}

1. Green, M. A., Emery, K., Hishikawa, Y., and Warta, W. (2009) Solar Cell Efficiency Tables (version 33), Progress in Photovoltaics: Research and Applications 17, 85-94.

2. Kalyanaraman, R., Duscher, G., Krishnan, V., Garcia, H., and Malasi, A. (2015) Hollow photovoltaic fiber, U.S. Patent No. 9,054,248.

3. Tachan, Z., Ruhle, S., and Zaban, A. (2010) Dye-sensitized solar tubes: A new solar cell design for efficient current collection and improved cell sealing, Sol Energ Mat Sol C, 94, 317-322.

4. Solyndra, Wholesale SOLAR, https://wrww.wholesalesolar.com/brands/solyndra, accessed 06/11/2018

5. Li, Y., Peterson, E. D., Huang, H., Wang, M., Xue, D., Nie, W., Zhou, W., \& Carroll, D. L. (2010) Tube-based geometries for organic photovoltaics, Appl Phys Lett 96, 243505-243508.

6. Gaudiana, R., Li, L., Chittibabu, K., Eckert, R., Montello, A., Montello, E., and Wormser, P. (2011) Photovoltaic fibers, US Patent No. 7,894,694.

7. Huang, H., Li, Y., Wang, M., Nie, W., Zhou, W., Peterson, E., Liu, J., Fang, G., and Carroll, D. L. (2011) Photovoltaic thermal solar energy collectors based on optical tubes, Sol Energy 85, 450-454.

8. Garnett, E. C. and Yang, P. (2008) Silicon nanowire radial p-n junction solar cells, J Am Chem Soc 130, 9224-9225.

9. Jung, J.-Y., Guo, Z., Jee, S.-W., Um, H.-D., Park, K.-T., Hyun, M. S., Yang, J. M., and Lee, J.-H. (2010) A waferscale Si wire solar cell using radial and bulk p-n junctions, Nanotechnology 21, 445303-445309.

10. Kelzenberg, M. D., Boettcher, S. W., Petykiewicz, J. A., Turner-Evans, D. B., Putnam, M. C., Warren, E. L., Spurgeon, J. M., Briggs, R. M., Lewis, N. S. and Atwater, H. A. (2010) Enhanced absorption and carrier collection in Si wire arrays for photovoltaic applications, Nat Mater 9, 239-244.

11. Kuo, C.-L., Wang, R.-C., Huang, J.-L., Liu, C.-P., Wang, C.-K., Chang, S.-P., Chu, W.-H., Wang, C.-H. \& Tu, C.H. (2009) The synthesis and electrical characterization of $\mathrm{Cu}(2) \mathrm{O} / \mathrm{Al}: \mathrm{ZnO}$ radial $\mathrm{p}-\mathrm{n}$ junction nanowire arrays, Nanotechnology 20, 365603-365610.

12. Yoon, H. P., Yuwen, Y. A., Kendrick, C. E., Barber, G. D., Podraza, N. J., Redwing, J. M., Mallouk, T. E., Wronski, C. R., and Mayer, T. S. (2010) Enhanced conversion efficiencies for pillar array solar cells fabricated from crystalline silicon with short minority carrier diffusion lengths, Appl Phys Lett 96, 213503-213506.

13. Dasgupta, P.K., Genfa, Z., Poruthoor, S.K., Caldwell, S., Dong, S. and Liu, S.Y. (1998) High-sensitivity gas sensors based on gas-permeable liquid core waveguides and long-path absorbance detection, Anal Chem 70, 4661-4669.

14. Eijkelenborg, M.A., Argyros, A., Barton, G., Bassett, I.M., Fellew, M., Henry, G., Issa, N.A., Large, M.C.J., Manos, S., Padden, W., Poladian, L. and Zagari, J. (2003) Recent progress in microstructured polymer optical fibre fabrication and characterisation, Opt Fiber Technol 9, 199-209.

15. Fink, Y., Ripin, D., Fan, S., Chen, C., Joannopoulos, J., and Thomas, E. (1999) Guiding optical light in air using an all-dielectric structure, J Lightwave Technol 17, 2039-2041.

16. Harrington, J. A. (2000) A review of IR transmitting, hollow waveguides, Fiber Integrated Opt 19, 211-227.

17. Johnson, S., Ibanescu, M.,Skorobogatiy, M., Weisberg, O., Engeness, T., Soljacic, M., Jacobs, S., Joannopoulos, J., and Fink, Y. (2001) Low-loss asymptotically single-mode propagation in large-core OmniGuide fibers, Opt Express 9, 748-779.

18. Kato, Y., Osawa, M., Miyagi, M., Abe, S., Aizawa, M., and Onodera, S. (1995) Loss characteristics of polymidecoated silver hollow glass wave-guides for the infrared, Electron Lett 31, 31-32.

19. Matsuura, Y. and Miyagi, M. (1998) Flexible hollow waveguides for delivery of excimer-laser light, Opt Lett 23, $1226-1228$.

20. Rabii, C. D., and Harrington, J. A. (1996) Optical properties of dual-core hollow waveguides, Appl Optics 35, 6249-625: 
21. Yan, M. and Mortensen, N. A. (2009) Hollow-core infrared fiber incorporating metal-wire metamaterial, Opt Express $17,14851-14864$.

22. Mohebbi, M., Fedosejevs, R., Gopal, V., and Harrington, J. (2002) Silver-coated hollow-glass waveguide for applications at $800 \mathrm{~nm}$, Appl Optics 41, 7031-7035.

23. Gopal, V., and Harrington, J. (2003) Deposition and characterization of metal sulfide dielectric coatings for hollow glass waveguides, Opt Express 11, 3182-3187.

24. Gopal, V. (2003) New dielectric coatings for low-loss hollow glass waveguides and bundles. Ph.D. Thesis, Rutgers, The state university of New Jersey, 52-54

25. Gopal, V., Harrington, J., Goren, A., and Gannot, I. (2004) Coherent hollow-core waveguide bundles for infrared imaging, Opt Eng 43, 1195-1199.

26. Mane, R. S. and Lokhande, C. D. (2000) Chemical deposition method for metal chalcogenide thin flms, Mater Chem Phys 65, 1-31.

27. Mayes, C., Schlichting, H., Krause, E., Oertel, H., and Gersten, K., (2003) Boundary-Layer Theory, Springer Berlin Heidelberg.

28. Lee, K. S. and El-Sayed, M. A. (2006) Gold and silver nanoparticles in sensing and imaging: Sensitivity of plasmon response to size, shape, and metal composition, J Phys Chem B 10, 19220-19225.

29. Hao, E. and Schatz, G. C. (2004) Electromagnetic Ỵelds around silver nanoparticles and dimers, J Chem Phys 120, 357-366.

30. Haes, A. J. and Van Duyne, R. P. (2002) A nanoscale optical biosensor: Sensitivity and selectivity of an approach based on the localized surface plasmon resonance spectroscopy of triangular silver nanoparticles, JAm Chem Soc 124, 10596-10604.

31. Taz, H. and Ruther, R. and Malasi, A. and Yadavali, S. and Carr, C. and Nanda, J. and Kalyanaraman, R. (2015) In situ localized surface plasmon resonance (lspr) spectroscopy to investigate kinetics of chemical bath deposition of cds thin films, J Phys Chem C 119, 5033-5039.

\section{ABOUT THE STUDENT AUTHORS}

Mikayla Ehrsam graduated from the University of Tennessee in May of 2018 with a Bachelor of Science in Mechanical Engineering and a minor in Reliability and Maintainability Engineering. She is currently working for Southern Nuclear. Humaira Taz is a PhD student studying Energy Science and Engineering at the University of Tennessee's Bredesen Center. Connor Carr graduated from the University of Tennessee in May of 2016 with a Bachelor of Science in Materials Science and Engineering. He is currently pursuing a $\mathrm{PhD}$ in Materials Science and Engineering at Northwestern University. Abhinav Malasi completed his PhD in Chemical Engineering at the University of Tennessee in May of 2016 and is currently looking for academic positions while taking time off in Europe.

\section{PRESS SUMMARY}

In order to construct such a solar device in a hollow polymer cylinder, one must be able to use chemical bath deposition to deposit homogeneous films with the appropriate microstructure. The objective of this project was to deposit a smooth, homogeneous silver film inside a hollow cylindrical structure using varying flow rates. The early stage film growth for very short deposition times was observed by the localized surface plasmon resonance of the silver nanoclusters via absorption spectra along the length of the tube. For longer deposition times, silver films formed and were analyzed for their morphology, thickness, roughness, and resistance using a combination of optical microscopy, scanning electron microscopy, and two-probe conductivity. The findings from this study showed that deposition under flow with different Reynolds numbers had a strong influence on the early stage and final morphology, and resistance of the deposited films. 


\title{
Feasibility of Man-Portable Power Generation Systems
}

\author{
Earl C. Allen and Nelson Fumo* \\ Mechanical Engineering Department, The University of Texas at Tyler, Tyler, TX \\ Student: eallen13@patriots.uttyler.edu \\ Mentor:nfumo@uttyler.edu*
}

\begin{abstract}
Electric power is the most common source of power for many portable devices used in outdoor recreation, emergency scenarios, and military applications. Batteries that have limitations regarding power and energy are currently meeting the power demand for most of these devices. There is a search to use alternative power sources for portable energy needs. This search has mostly been researched particularly for the military to satisfy functions necessary for soldiers. However, any alternative that would provide electricity in an efficient and timely manner is useful in many applications beyond the military. The feasibility of man-portable power generation systems using thermal energy from any kind of fuels needs to be studied further, to understand if they are truly an option for situations where batteries are currently used. In this study, the feasibility of fueled power systems is investigated. Commercially available small power systems show that they are not small enough to satisfy the definition of man-portable power system. However, power systems working on an internal combustion engine show to be a better option when compared to batteries and fuel cells
\end{abstract}

\section{KEYWORDS}

Fueled Power Generation; Man-portable Power; Power Density; Energy Density.

\section{INTRODUCTION}

There is a large need for power systems in today's age of technology, with all of the different devices that are reliant on some sort of electrical power source. ${ }^{1}$ In many cases, these devices are able to be connected to large-scale power systems or onto a power grid. However, there are many scenarios where connecting to a large-scale power system or onto a power grid is not possible. Because these scenarios exist, there is a need for portable power systems. These systems are designed to be used in scenarios such as, soldiers in combat, use in third world countries, and camping trips. Currently, there are already many systems that are small portable systems. The majority of these systems focus on the ability to charge cell phones. This is a very large market and is very important, but it does not mean that man-portable systems have to be restricted to this one particular function.

When someone is in a remote location and needs power, there are a couple of different types of systems that can be used. One example of this is an electric storage system, such as batteries or a person using a device to generate power. Electric storage systems are commonly used today for portable systems. The move to power generation systems for these applications needs to be further understood and further developed. ${ }^{2}$

All power systems have fuels that have certain characteristics. The key to creating small power systems is to be able to understand these characteristics and use those well in designing a portable power system. In order to maximize energy in as small of a system as possible, there has to be an understanding of energy density. There are two types of energy density specific energy, also known as gravimetric energy density, and volumetric energy density, generally referred to as energy density. Specific energy is simply energy per unit of mass, whereas volumetric energy density is energy per unit volume. ${ }^{3}$ These values are related to each other by density, and because of this one of these values may become more important in certain situations.

In order to create smaller scaled power systems there are some key concepts that need to be understood about fuels as well as the many options for fuels. There are a variety of fuel types and energy systems to use each of those fuel types. For the most part, these systems can be converted into small-scale systems in order to be able to be a portable system with the downside of producing less power. Fuels also have heating values. There are different measures for heating values, high heating value and low heating value. High heating value is the amount of heat that is released by a fuel when the temperature starts at $25^{\circ} \mathrm{C}$, is burned, then returns to $25^{\circ} \mathrm{C} .{ }^{4}$ Low heating value is the amount of heat released when the fuel starts at $25^{\circ} \mathrm{C}$, is burned, and then returns to $150^{\circ} \mathrm{C} .{ }^{4}$ These are each used in specific situations, high heating value is used when liquid water exists as a product at the end of the combustion process. Low heating value is used when water vapor exists as a product at the end of the combustion process. There are a wide variety of fuels that can be used in different systems, all of which fit in a few categories including fossil fuels, gaseous fuels, and renewable fuels. 
Another important aspect of power systems is the actual system itself. When the system is chosen it will help to limit the options for fuels that are usable with that system. The main value for being able to understand the usefulness of a system is the power density, which is the rate of energy flow measured in power per unit of volume. ${ }^{5}$ In this particular case though, there will be another limiting factor on systems and that is the ability to fit in the category of man-portable.

\section{Man-Portable Power}

To be able to understand what man-portable is, there first must be an understanding of portable. Portable is something that can be transported by people. ${ }^{6}$ The problem with this definition, when talking about power systems that need to be moved, is that by definition a portable system could take multiple people to move. For example, the Yamaha EF2000iSv2 Portable Inverter is a portable power system that weighs $44.1 \mathrm{lbs}$, but it may take multiple people to move it. ${ }^{7}$ There may be a person strong enough to move this portable system by themselves, but they will not be able to carry many other items with them at the same time. This would not be a viable option for someone who is in many scenarios such as someone backpacking or a soldier on a mission. Because of these scenarios, there is a need for man-portable power systems. Man-portable is defined by the Department of Defense as being a system that is less than 14 kilograms (31 pounds). The Department of Defense also describes man-portable power as, "equipment which can be carried by one man over long distance without serious degradation of the performance of normal duties". 8 While the Yamaha EF2000iSv2 Portable Inverter Generator may be a very useful power system in a scenario where a vehicle can be used, it would not be optimal in a scenario where just people would be required to transport it. With this definition of man-portable in mind, the previous example of the Yamaha EF2000iSv2 Portable Inverter Generator would be considered portable but would not fit the definition of man-portable. ${ }^{7}$

The definition of man-portable allows for an understanding that when creating a small power system, the greatest limitation is weight. Because the limiting factor based on that definition is weight, a design is not limited to the amount of power it can produce by definition. This means that there is a large need for understanding how to maximize power output base on weight. In a practical sense, though it is not defined based on the definition of man-portable, there is a need to keep within a reasonable volume. This means there needs to be a balance struck when choosing fuels. Just because there is a need to minimize weight does not mean that it may be necessarily reasonable to choose the fuel with the greatest specific energy. Based on the definition of man-portable the idea of what is reasonable for the amount of volume for a man-portable power system is left up to the engineer designing the system. The engineer may be helped in that decision by many constraints based on a specific application in which they are designing the system for. In the case of man-portable power, minimizing volume is still a priority. Since both of these are of huge importance for a man-portable system, there are a few fuels that would be considered the best fuels for man-portable applications. When discussing fuels later these will be very important values in selecting the best possible fuels for a man-portable system. ${ }^{7}$

\section{Ragone Plot}

When trying to understand energy storage systems it is helpful to be able to understand the options available and their characteristics through visuals. A well-known way to do this is through something called Ragone plots. A Ragone plot represents power density versus energy density of energy storage systems on a logarithmic scale. ${ }^{9}$ Both characteristics are very important when considering man-portable systems because they both are defined per unit of mass.

It is important to understand what specific energy and specific power mean in terms of production. If a system has a high specific energy, then it can produce energy for long periods of time. ${ }^{10} \mathrm{~A}$ system that has a high specific power would mean that the system can produce a lot of energy at a small interval of time for its weight. ${ }^{11}$ For instance, in the case of batteries, they have a high specific energy but they also have a relatively low specific power. Because of those properties, batteries are typically used to power systems that need to be powered for long periods of time, but they do not necessarily power systems that require a lot of power to run. It makes sense that it would be optimal to maximize both the specific power and specific energy for man-portable systems.

Most Ragone plots only show energy storage systems. However, the Ragone plot in Figure 1 also shows that combustion engines have more specific energy and more specific power than all the storage systems shown in the graph. This means that there is a very good potential that power systems have more specific power and more specific energy. ${ }^{5}$ This is a good reason to continue seeking out man-portable power generation systems as an option over man-portable energy storage systems. 


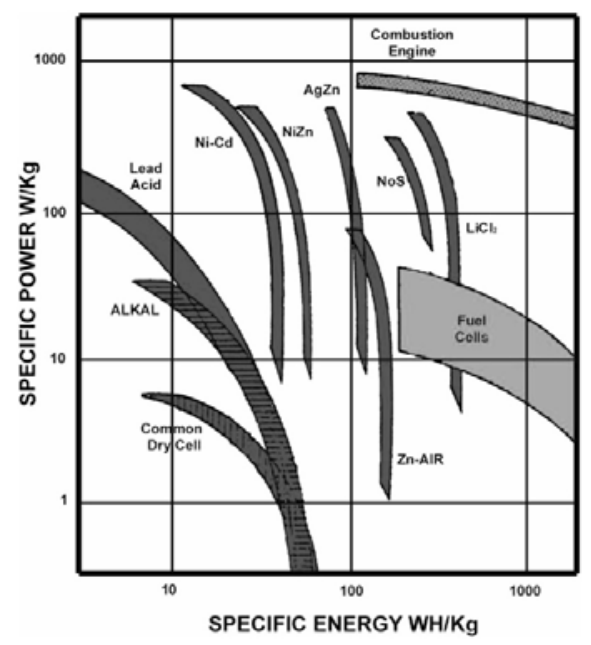

Figure 1. Example of a Ragone plot that includes many energy storage systems as well as combustion engines. ${ }^{12}$

\section{Fueled Power Systems}

There is a large amount of various types of small-scale power systems. Some of these small-scale power systems include: renewable energy systems, micro turbines, micro engines, Stirling engines, and Rankine Cycle systems. The reasons for using any of these systems should be weighed out in any design scenario. For man-portable the power system chosen should be usable no matter the environment. A man-portable power system should emphasize the ability for a lightweight design along with allowing for a small volume design. Preferably, a man-portable system would produce a relatively small amount of noise and produce a minimal amount of exhaust. ${ }^{13}$ If possible a man-portable power system would produce a low temperature profile as well, this would allow for it to be used for military applications. These many properties of any particular power system should be weighed out before choosing a particular way of producing man-portable power.

Renewable energy is a type of power source that is currently becoming very popular. For the purposes of this paper, Biomass will be considered separately even though it is considered renewable energy. This is because biomass can be interchanged with other fuel sources such as fossil fuels. Most renewable energy sources need a power system that is specific to that energy source. The best attribute of renewable energy, such as, wind, solar, and hydro power, is that it is virtually infinite. Wind and hydro power have not yet shown themselves to be a great source of portable power and most likely won't, due to the nature of them needing large amounts of fluid to be able to generate adequate power. Solar Energy, however, has shown itself to be a great source of portable power. There are already many portable systems that use solar energy and a battery to create energy on a small portable scale. Some of these, such as the Goal Zero Venture 30 Solar Recharging Kit, even fit the definition of man-portable because it weighs only 1.56 lbs. ${ }^{14}$ Though the Goal Zero Venture 30 Solar Recharging Kit is specifically designed to recharge tablets and phones it would still be considered a man-portable power system. A great thing with the solar systems is that their power source is infinite, along with the fact that it does not need to be stored along with the power system. This limits the customer costs to simply buying the power system that they want and putting that system to use. It also keeps that person from having to carry some other form of fuel along with the power system they have creating extra weight during transportation. The limiting factor to a solar power system is the fact that the sun is not always accessible. There are many scenarios where a solar powered system would not be able to generate any power, and this severely limits its ability to be a great option for a man-portable power system. Scenarios such as natural disaster settings and night missions for soldiers make it necessary for other forms of power systems to be explored.

A form of small-scale engines used to produce power has become popular, this market is Portable Generators. Portable Generator Manufacturers' Association (PGMA) defines portable generators as engine driven power generators designed for portability. ${ }^{6}$ PGMA includes in their definition that generators designed to be transported via vehicle are not considered a portable generator. ${ }^{6}$ There is a large variety of portable generators that can be located online that include previously mention Yamaha EF2000iSv2 Portable Inverter Generator ${ }^{7}$ and another called the Pulsar 1200W 2-Stroke Generator. ${ }^{15}$ Both of these are portable power systems coming in at $44.1 \mathrm{lbs}$ and $37.5 \mathrm{lbs}$ respectively. There are many other portable generators that come in at similar weights; the problem is that both of these systems do not fit the definition of man-portable power systems. The fact that there is a large industry that has many products that have not met the man-portable definition would lead to a belief that micro engines may not be a good option for man-portable power. Recently, however, there has been a portable generator that meets the man-portable definition called the GenSmart 1000. The GenSmart 1000 weighs $12 \mathrm{lbs}$ and has a large amount of power output 
for its weight. ${ }^{16}$ The GenSmart 1000 also uses methanol as opposed to what most portable generators use, gasoline. ${ }^{16}$ This may mean that in order to create man-portable generators fuels may have to be studied more deeply. A downside to micro engines in general is that there must be some type of fuel to accompany the engine; consequently this fuel creates extra weight. Due to the extra fuel needing to be carried, the user also has to determine how much fuel they will need for their trip and find a way to carry that with them. This severally limits how long a micro engine could be used for a trip. The advantage of using micro engines is when the user needs energy it is easy to start up the micro engine and use just as much fuel as needed for that particular application. A further study of fuels specifically for micro engines could lead to a break through and allow micro engines to be a feasible idea for man-portable power.

A relatively new power system altogether is microturbines. ${ }^{17}$ When talking about micro turbines, the term micro does not refer to physical size but to the power capacity. Microturbines are currently built for distribution power purposes, which are primarily large-scale systems. ${ }^{18}$ This technology has a high specific power and high specific energy ${ }^{19}$, due to this , micro turbines could be an interesting option for portable power generation systems. Microturbines do not currently seem like a viable option for manportable power generation systems, because they have not yet proven that they can be made as small-scale systems.

Stirling engines are an interesting option for use, as a man-portable power system. There are many advantages to using Stirling engines, the main being that they are maintenance free and use fuel externally. ${ }^{20}$ Meaning that nearly any fuel could be used to power the system and, theoretically, the fuel used could be changed on a situational basis. Another major advantage to Stirling engines is that they are considered quiet machines. The fact that Stirling engines are quiet allows them to be a good option for use on military missions. It also makes Stirling engines a good option for camping if there were some need for the use of a power system overnight. The major downside to Stirling engines is their relatively low specific power compared to other power systems. ${ }^{19}$ This means that relative to other system there is not a possibility of getting a large amount of power in a man-portable system. Additionally, Stirling engines have a long start time which means if for some reason there was an urgent need for power then it would not be possible with a Stirling engine. ${ }^{19}$ When considering man-portable systems, the fact that Stirling engines have a low specific power makes it necessary to highly consider other options.

Fuels

After a type of power generation system has been chosen, the fuel to be used needs to be chosen. There are a variety of options available, but these options need to be understood in depth before making that decision. When talking about man-portable power systems, there will be some very specific properties that need to be discussed to make a good decision.

There are different types of fuels. The type of fuel used is very much dependent on the type of power generation system being used. For instance, renewable energies such as solar and wind, need specific power generation systems to generate power. Also, internal combustion engines are limited to types of fuels. However, external combustion systems can use nearly any type of fuel. Regardless it is good to have a proficient understanding of what type of fuel would be good to use in any specific power generation system.

Fuels are generally grouped into subsections of renewables and fossil fuels, each of these have even smaller subsections. For example, fossil fuels can be broken down into coals, petroleum, and gaseous fuels. In this case the fuels will instead be broken down into their states at room temperature such as gas, liquid, or solid. This is because, for man-portable power, it is crucial to know how the fuel would be carried as opposed to knowing the kinds of fuels.

Table 1 shows a list of common fuels. These fuels except for wood are fossil fuels; wood is considered a biomass fuel. This table gives some typical characteristics as well as their phase state and various properties relevant to this study. The properties shown in this table are high heating value, energy density, burn temperature, and the adiabatic flame temperature of the fuels. As a refresher, the high heating value is the amount of heat released, when a fuel starts at $25^{\circ} \mathrm{C}$ and then returns to $25^{\circ} \mathrm{C} .{ }^{4}$ Adiabatic flame temperature is the theoretical temperature that can be released if no heat is lost to the environment and no heat is added from the environment. ${ }^{21}$ These properties are relevant to determining what kind of fuel to use for a man-portable application. The main two properties that will be consistently seen with the same importance are the high heating value and energy density. These two values need a balance to be struck. As previously discussed, these values are related by density. Table 1 shows that there should not be a fuel chosen based on either the high heating value or energy density alone. A balance needs to be struck between both the weight and volume that is taken up for a fuel. For example, hydrogen has the highest heating value of any of the fuels listed but it also has a very low energy density. This means, while it may have a lot of energy for the amount of mass, it will take up a lot of space. With that being said, hydrogen is probably not a viable option as a fuel, for a man-portable system. 


\begin{tabular}{|c|c|c|c|c|c|}
\hline Fuel & State & $\begin{array}{l}\text { High Heating } \\
\text { Value } \\
\quad(\mathrm{kJ} / \mathrm{kg})\end{array}$ & $\begin{array}{l}\text { Energy Density } \\
(\mathrm{kJ} / \mathrm{L})\end{array}$ & $\begin{array}{l}\text { Burn } \\
\text { Temperature } \\
\qquad\left({ }^{\circ} \mathrm{C}\right)\end{array}$ & $\begin{array}{c}\text { Adiabatic Flame } \\
\text { Temperature } \\
\left({ }^{\circ} \mathrm{C}\right)\end{array}$ \\
\hline Anthracitic Coal & Solid & 27000 & 36450 & 600 & 2180 \\
\hline Bituminous Coal & Solid & 23250 & 0.026 & 454 & 2172 \\
\hline Diesel & Liquid & 44800 & 37184 & 210 & --- \\
\hline Ethane & Liquid & 51900 & 28246 & 515 & 1955 \\
\hline Ethanol & Liquid & 29700 & 23278 & 365 & 2082 \\
\hline Gasoline & Liquid & 47300 & 33867 & 246 & 2138 \\
\hline Hydrogen & Gas & 141790 & 0.00001 & 500 & 2254 \\
\hline Kerosene & Liquid & 46200 & 38346 & 295 & 2093 \\
\hline Lignite Coal & Solid & 16300 & 12015 & 526 & --- \\
\hline Methane & Liquid & 55530 & 23529 & 580 & 1963 \\
\hline Methanol & Liquid & 23000 & 17855 & 470 & 1949 \\
\hline Natural Gas & Gas & 71525 & 37300 & 580 & 1960 \\
\hline Propane & Gas & 50350 & 29449 & 455 & 1980 \\
\hline Wood & Solid & 17400 & 9000 & 300 & 1980 \\
\hline
\end{tabular}

Table 1. Properties of some well-known fuels.

The relationship between high heating value and energy density for the fuels given in Table 1 is shown in Figure 2 . Each fuel is plotted on the graph showing how it relates in terms of high heating value and energy density. Figure 2 shows that many fuels are similar in terms of their energy density to high heating value ratio. It can also be seen that the best fuel, in terms of energy density and high heating value, is Natural Gas. However, a large problem with a gas fuel is the way they need to be transported. Gas fuels are stored at high pressures this can be a safety issue for man-portable power systems. Also, the containers in which those gases are stored in must be very thick and bulky in order to hold those gases at high pressure. With the bulky containers, it would be worth looking at other options that would be able to produce a satisfactory amount of energy to the amount of weight including the container.

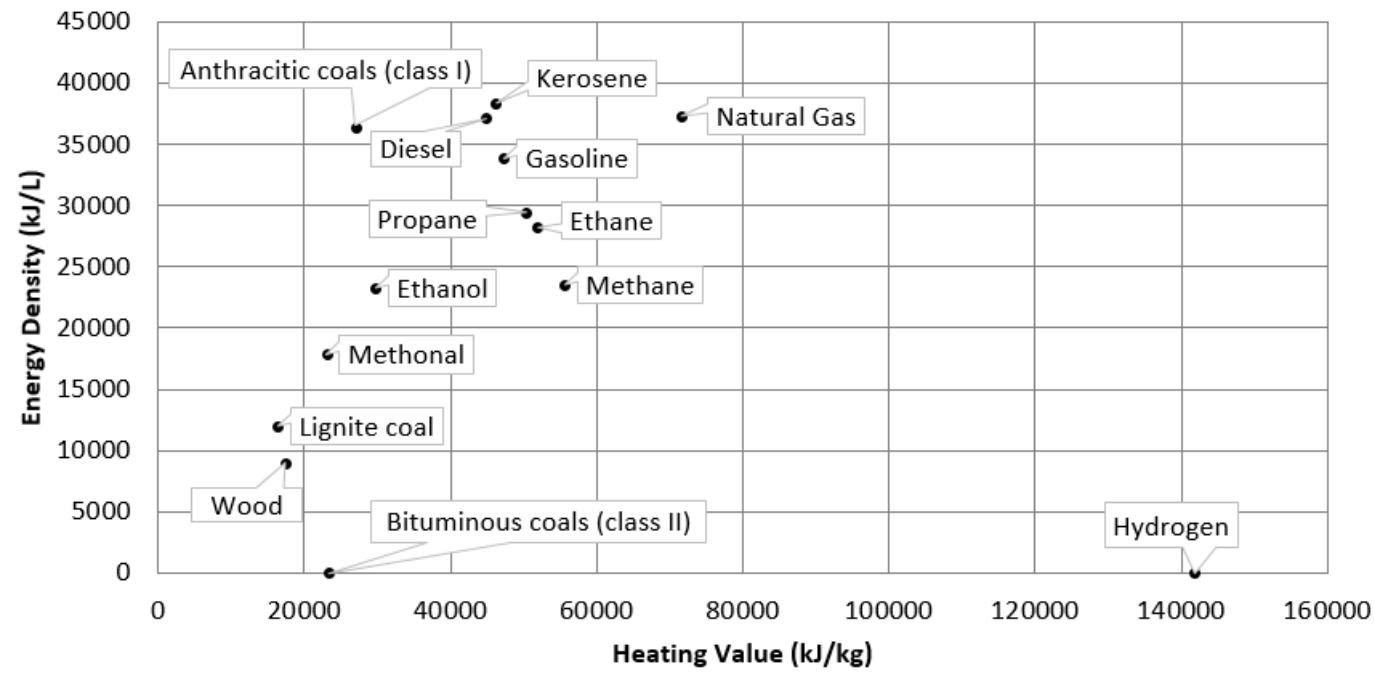

Figure 2. Fuels ratio of energy density and heating value.

Solids are another option for fuels. The main solid fuels that are used are coal and wood. Coals are generally used in large-scale facilities and are very hard to burn. As it can be seen from Table 1, the burn temperatures of the three types of coal are rather high, with the lowest burn temperature being $454^{\circ} \mathrm{C}$ for Lignite Coal. Coal would also be a rather difficult fuel to carry. The other solid option is wood. As shown in Figure 2, wood does not have a very good heating value or energy density, relative to other options. However, in the case of an external combustion engine, wood might be a good option as an alternative source of heat. This is because somebody who is in an area, particularly a campsite, could harvest wood for use as fuel to burn. Even though this is a feasible option, wood is still not a good selection when a choice is given. 
Liquids make up the majority of fuels. They are a very good option, because they are easy to carry at atmospheric pressure with a simple container. This is very crucial for man-portable situations. The two best options for liquid fuels in terms of their heating values and energy density are both kerosene and diesel fuel. Both kerosene and diesel are very close to each other in terms of heating value and energy density. Because they are comparable, it is worth noting that the burn temperature for kerosene is $295^{\circ} \mathrm{C}$ as compared to $210^{\circ} \mathrm{C}$ for diesel. That means that between the two fuels it is probably worth leaning towards diesel just because it is the easier fuel to burn. According to the Occupational Safety and Health Administration (OSHA), liquid fuels should be handled and transported in portable fuel containers. The portable containers should be metal, have tight closures (with screw or spring covers). ${ }^{22}$ They should also be equipped with spouts to allow pouring without spilling. If those fuel containers are used with portable generators, the containers should be directly attached to the generator frame to prevent static build-up and reduce risks of explosions.

\section{Current Portable Fuel Powered Systems}

Currently man-portable fuel powered systems do not have a large industry. However, there is a large industry for portable fuel powered systems, that being portable generators. This is a large industry that already exists and is good to be used to help understand what can be accomplished with man-portable fuel powered systems. Also, portable generators have man-portable fuel powered systems within them. Table 2 gives a list of 14 portable generators that are commercially available along with their specifications. Some of the specifications required basic calculations to be reached; those calculations were based on given information about the product. For example, volume was not given, but length, height, and width were, so those values were used to calculate volume.

One of the portable generators in Table 2 meets the definition of man-portable that was stated to be as a system that weighs less than 14 kilograms (31 pounds). This gives an idea of what is possible with man-portable fuel powered generators. This is a relatively limited view of what can be accomplished with man-portable fuel power systems because all the systems in Table 2 are internal combustion engines.

\begin{tabular}{|c|c|c|c|c|c|c|}
\hline Company & $\begin{array}{l}\text { Power } \\
\text { Output } \\
\text { (W) }\end{array}$ & $\begin{array}{l}\text { Weight } \\
\text { (kg) }\end{array}$ & $\begin{array}{l}\text { Specific } \\
\text { Power } \\
\text { (W/kg) }\end{array}$ & $\begin{array}{l}\text { Volume } \\
\qquad\left(\mathrm{m}^{3}\right)\end{array}$ & $\begin{array}{c}\text { Gravimetric } \\
\text { Energy Density } \\
\text { (Wh/kg) }\end{array}$ & Fuel \\
\hline Yamaha & 2000 & 20.0 & 100 & 0.0623 & 210 & Gasoline \\
\hline Pulsar & 1200 & 17.0 & 70 & 0.0368 & 225 & Dual Fuel \\
\hline Honda & 2000 & 20.7 & 97 & 0.0630 & 290 & Gasoline \\
\hline Sportsman & 4000 & 46.3 & 86 & 0.1284 & 432 & Propane \\
\hline $\begin{array}{l}\text { Champion } \\
\text { Power }\end{array}$ & 4000 & 48.6 & 82 & 0.1328 & 494 & Gasoline \\
\hline A-iPower & 4000 & 47.7 & 84 & 0.1189 & 503 & Gasoline \\
\hline Honda & 1000 & 13.0 & 77 & 0.0413 & 230 & Gasoline \\
\hline Honda & 3000 & 35.4 & 85 & 0.1155 & 212 & Gasoline \\
\hline Honda & 6500 & 81.3 & 80 & 0.2066 & 400 & Gasoline \\
\hline Honda & 3000 & 32.2 & 93 & 0.0857 & 437 & Gasoline \\
\hline $\begin{array}{l}\text { Briggs \& } \\
\text { Stratton }\end{array}$ & 8000 & 127.6 & 63 & 0.4406 & 353 & Gasoline \\
\hline Generac & 5500 & 83.0 & 66 & 0.3163 & 414 & Gasoline \\
\hline $\begin{array}{l}\text { Smarter } \\
\text { Tools }\end{array}$ & 6500 & 90.8 & 72 & 0.2794 & 413 & Dual Fuel \\
\hline Ryobi & 5500 & 88.1 & 62 & 0.2738 & 351 & Gasoline \\
\hline
\end{tabular}

Table 2. Characteristics of commercially available portable generators.

External combustion engines could be a design choice for portable generators as well as man-portable fuel powered systems. The main draw towards external combustion engines would be their versatility for fuels. They can use nearly any fuel that can be obtained. This is very useful in man-portable situations. If somebody is camping and they run out of the fuel they can use wood to help generate power. Another situation where this is beneficial is in a military setting. For example, if a soldier is on a mission and they run out of fuel, any other fuel available could be used, since a substitution for a different fuel will still produce the thermal energy needed by the power system. 
Figure 3 is built based on Table 2 and Figure 1 and is given to illustrate a relative comparison of different options for sources of power. Figure 1 shows that combustion engines have the potential to have both more specific power and more specific energy than current batteries and fuel cells. From Figure 1, regions 1, 2, and 3 in Figure 3 are shown for batteries, fuel cells, and combustion engines respectively. For comparison purposes, the current portable power generation systems were plotted on the logarithmic scale plot shown in Figure 3 and are represented by region 4. This shows that the current commercially available portable power generation systems are not yet meeting their theoretical capabilities. However, they are close to having more specific power than current batteries and they provide nearly as much specific energy as fuel cells. This means, that the current portable power generation systems already provide the best of both worlds, if there is a need for a lot of energy and a lot of power in a lightweight system. Based on Figure 1, since commercially available portable systems work with the same technology (thermodynamic cycles) as internal combustion engines, there is an expectation that portable systems will be able to provide more power and energy per unit of weight going forward in the future. This expectation is not being met, most likely because of their size. Power generation systems, based on internal combustion engines, have better efficiency for large capacity than a unit with less capacity. This decrease in efficiency has a negative effect on both the specific power and specific energy. Finding a way to create more efficient small scale fuel powered systems could allow for region 4 to get much closer to region 3 .

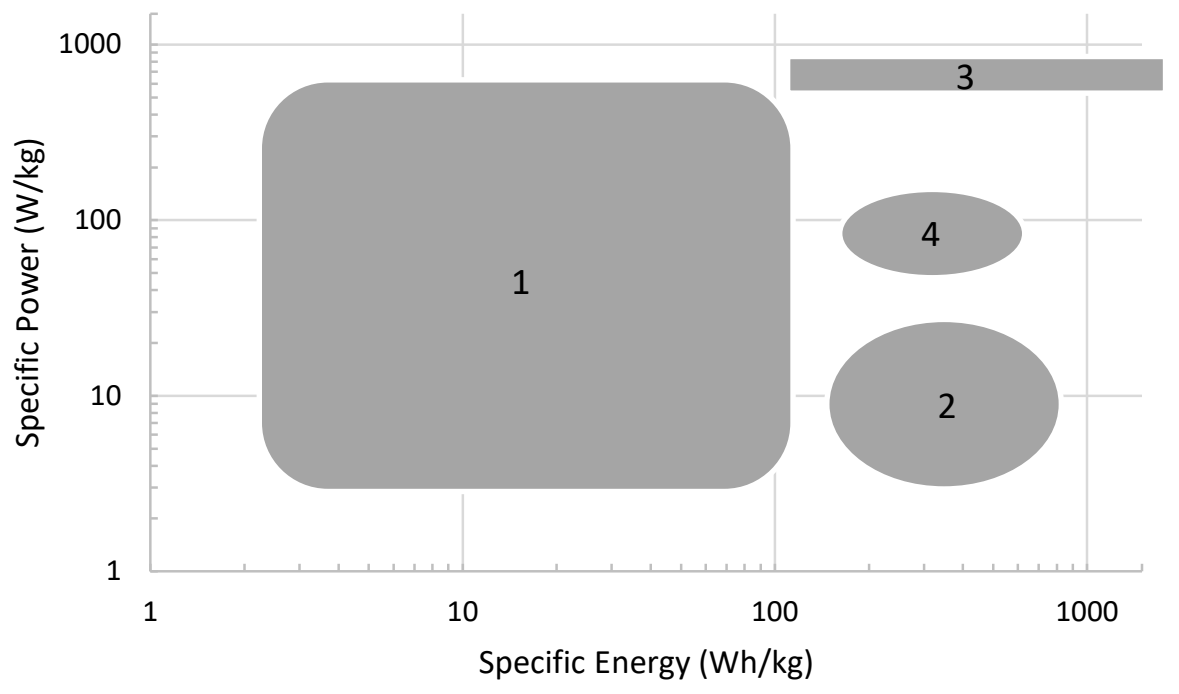

Figure 3. Ragone plot showing how commercially available Portable Power Systems compare to batteries and fuel cells and combustion engines. Number 1 refers to batteries, 2 refers to fuel cells, 3 refers to combustion engines, and 4 refers to current commercial portable fuel powered systems.

\section{Efficiency and Autonomy}

For the concept of a man-portable fuel power generator, the system is comprised of the engine, the fuel, and the container for the fuel. The volume, and consequently the weight, of the container is dependent on the amount (weight) of the fuel. Therefore, for the purposes of this study, the weight of the container has been set as 0.29 times the weight of the fuel. This value was obtained by weighing water in a stainless-steel tumbler, then getting the weight ratio of the empty container to the container full of water. The engine is a constant weight that by itself creates no energy. Since there is no energy created, when the power generation system is comprised of just an engine, the specific energy at that weight is zero. In the case of Figures $\mathbf{4}$ and $\mathbf{5}$ the specific weight of the engine was assumed to be $2.38 \mathrm{~kg}$. This weight was defined based on a need for $200 \mathrm{~W}$ (value assumed to be needed for radio communications in the military ${ }^{23}$ ) and a specific power of $84 \mathrm{~W} / \mathrm{kg}$ (assumed based on the average specific power of the portable power generation systems in Table 2). Once the fuel is introduced to the system there is specific energy. The total specific energy of the entire system is based on how much fuel is carried and therefore how much fuel the engine can use. That fuel is dependent on how much can be carried for the specific situation. This concept is shown in Figures 4, 5, and 6.

Figure 4 shows a comparison of how four different fuels affect the specific energy of a fuel power generation system. For this graph a fuel-to-electricity efficiency of the system was assumed to be $20 \%$. Also, a specific power of $84 \mathrm{~W} / \mathrm{kg}$ was assumed based on the average specific power of the portable power generation systems in Table 2 . The four fuels were chosen since propane, gasoline, and diesel can be found in portable power generation systems currently. Kerosene was chosen because from Figure 2 it can be noticed that it has a very good specific energy and specific power. As shown in Figure 4, these fuels provide a similar specific energy to the system when the same weight is carried out (at zero specific energy the weight is related to the weight of the system without any fuel). However, this figure also shows the importance of increasing the amount of fuel on the specific energy 
of the system. The first 50\% increase, in specific energy, happens within the first three kilograms of fuel added to the system. After the entire system reaches six kilograms the increase in specific energy per kilogram of fuel added is significantly less. In other words, the most significant change in specific energy increase come in the first mass of fuel and there is a much smaller impact of specific energy as more fuel is added to the system.

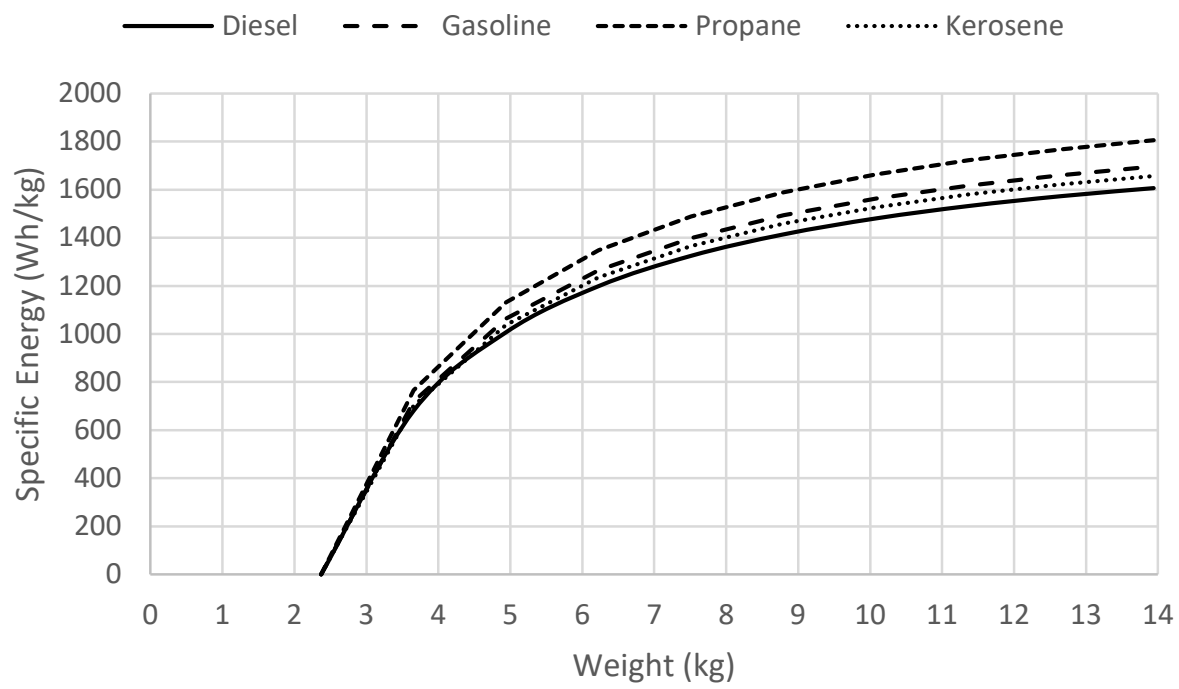

Figure 4. Comparison of four different fuels showing how much specific energy they can create with a standard engine based on the weight of the fuel. The specific power was set has $84 \mathrm{~W} / \mathrm{kg}$ and efficiency was set as $20 \%$.

Figure 5 illustrates the importance of the fuel-to-electricity efficiency of the system. This is based on the concept that the engine cannot create energy without fuel and the specific energy is dependent on the amount of fuel carried. This figure confirms the impact of the first kilograms of fuel carried for the system. As expected, the curves are proportional to each other throughout. This means that what was said to be true in Figure 4 previously, is true for all efficiencies.

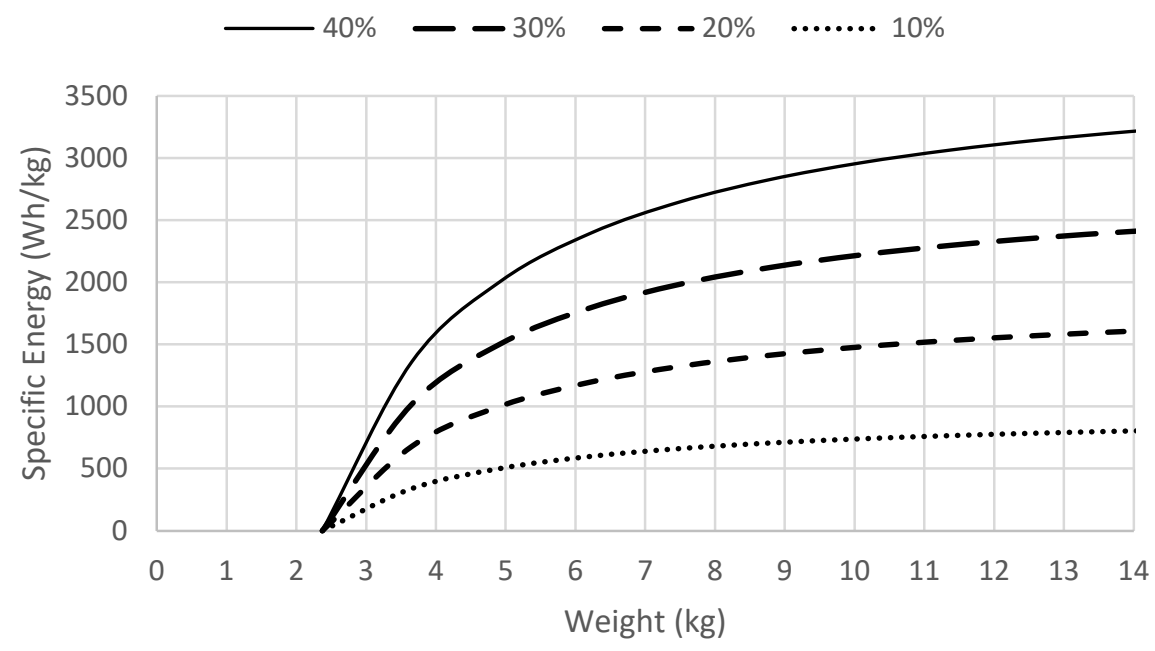

Figure 5. Comparison of fuel-to-electricity efficiency on an engines specific energy. These curves are based on the total weight of the system, and assumes specific power of $87 \mathrm{~W} / \mathrm{kg}$ and diesel fuel. Also, the weight of the container is 0.29 times the weight of the fuel.

Systems in Table 2 have specific power in a range of about $70 \mathrm{~W} / \mathrm{kg}$ to $100 \mathrm{~W} / \mathrm{kg}$, for portable power generation systems.

Figure 6 was created based on this range at intervals of $10 \mathrm{~W} / \mathrm{kg}$, to show the impact of specific power on the specific energy of the system. The difference in the specific power can be seen when specific energy equals zero, this is due to the change in specific power creating a change in the engine weight required. For example, to get $200 \mathrm{~W}$ of electricity, with a $100 \mathrm{~W} / \mathrm{kg}$ system only a 2 $\mathrm{kg}$ engine would be required. However, to produce the same power of $200 \mathrm{~W}$, a $70 \mathrm{~W} / \mathrm{kg}$ engine would weigh $2.86 \mathrm{~kg}$. This has a significant impact on the specific energy of the system when the weight of the system is reduced. The larger the total weight of 
the system, the less impact specific power has on specific energy. At a total weight of four kilograms there is about a $25 \%$ difference in specific energy for an $80 \mathrm{~W} / \mathrm{kg}$ engine and a $70 \mathrm{~W} / \mathrm{kg}$ engine. However, at $13 \mathrm{~kg}$ there is only about a $5 \%$ difference in specific energy.

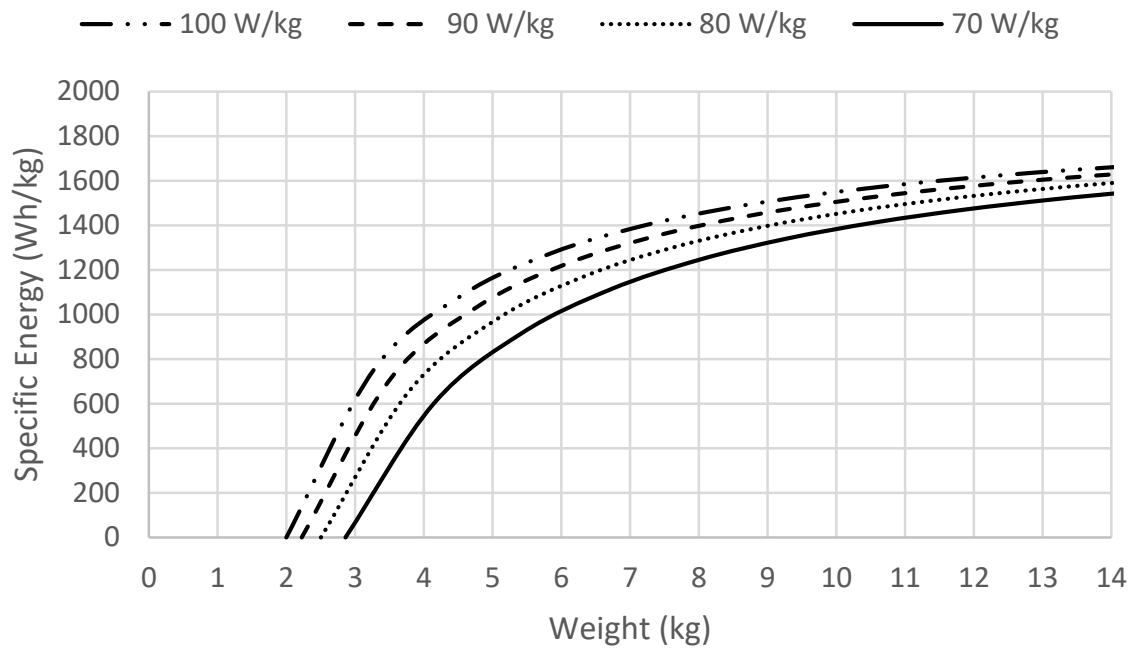

Figure 6. Comparison based on different specific power of the system. Diesel fuel and a fuel-to-electricity efficiency of $20 \%$ were assumed for this graph.

Figure 7 is a graph created to show the autonomy of operation in a portable fuel powered system based on the weight carried. The curves shown in Figure 7 compare the run time based on the efficiency of the engine. These curves were created by multiplying the specific energy by the specific power (specific power being assumed as $84 \mathrm{~W} / \mathrm{kg}$ ) and this product then multiplied by the mass of fuel at specific points to get the curves.

As seen from Figure 7, the first six kilograms define a slight parabolic curve. After six kilograms, the trend of the curves has a more linear path. The time of autonomy does not begin to be a reasonable amount for military applications until after about three kilograms. Once again, the time of autonomy is proportional to the efficiency of the system. Similar to how increasing the efficiency of the system creates a greater specific energy, it also allows for an increased time of autonomy for the system.

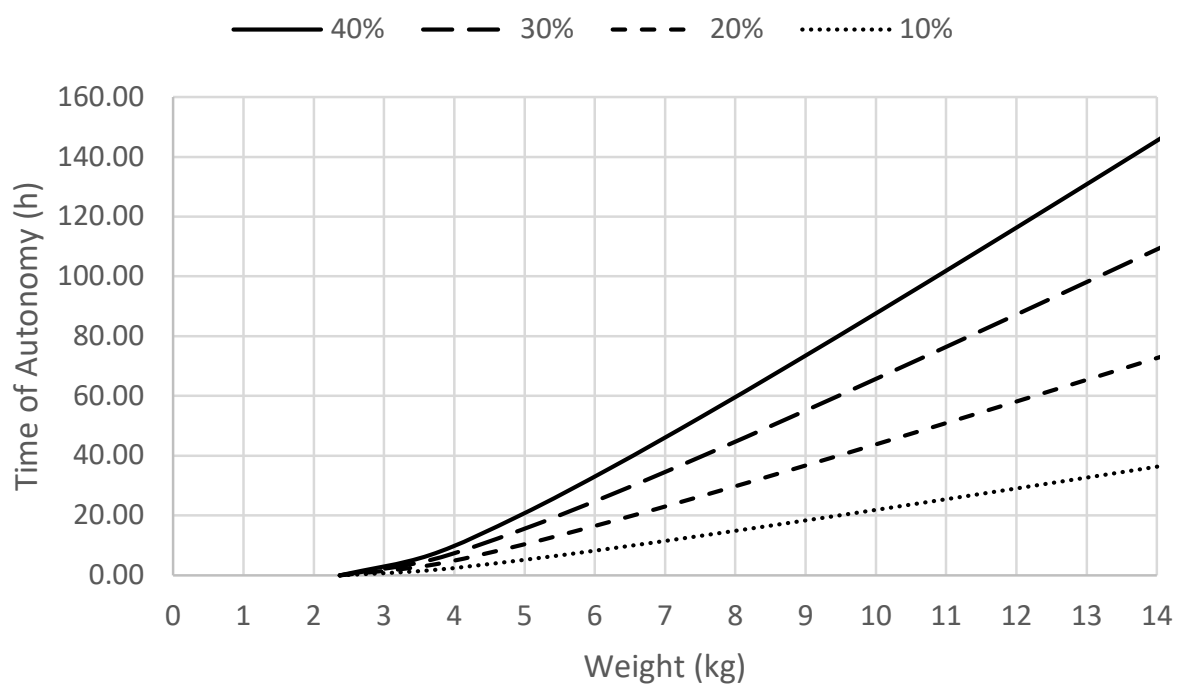

Figure 7. Time of system operation autonomy based on the weight of the entire system. Based on the efficiencies discussed in Figure 5 and a specific power of 84 $\mathrm{W} / \mathrm{kg}$.

Comparing Figure 4, 5, and 6 it is obvious that the fuel choices, the efficiency, and the specific power of an engine all influence the specific energy of a man-portable fuel power system. Occasionally the impact that those three characteristics can have on specific energy is based on weight. For instance, if there was a need to carry closer to 14 kilograms of fuel then there would be an 
impact on specific energy by changing the fuel type, than there would be by choosing an engine with a higher specific power. However, if the situation called for carrying less weight such as a total of five kilograms, then having a system with a higher specific power would have a greater impact. The specific energy of the system is proportional to the efficiency of the engine and would have a proportional impact on specific energy no matter the weight of the system. The amount of fuel that would need to be carried would be dependent on how much time the system needs to be run as shown in Figure 7.

Currently batteries are expected to allow a soldier to make it through a 72-hour mission without ever losing power. ${ }^{23}$ Currently soldiers are carrying 7.7 kilograms (17 pounds) of batteries to allow them to make it through 72 -hour missions. ${ }^{24}$ In Figure 7, at a $40 \%$ efficiency and 8 kilograms, there is only a 60 -hour run time. This means that, based on the estimations made, there needs to be more advancements made for small scale engines for them to be able to replace batteries on the battlefield.

For the man-portable power generation systems to meet the energy needs that the batteries are currently fulfilling the system would weigh about 14 kilograms at a 20\% efficiency. For the best-case scenario from Figure 7, a $40 \%$ efficiency, the system would weigh about 9 kilograms to run for 72 hours at 200 Watts. For man-portable power systems to be feasible to take over batteries in military applications the systems need to be even more efficient than they currently are, along with having more specific power. Trying to reduce the size of the current systems while maintaining the current efficiencies would help increase the time of autonomy to weight ratio. Getting man-portable power generation systems to be a feasible alternative would be beneficial because the systems would not need to be recharged or operated as a one-time use system like current batteries. When the soldiers need the power they simply put fuel in the system and allow for that to power their communication systems.

\section{CONCLUSION}

There is a need for small-scale systems that generate or store electricity for use in man-portable situations, including military applications, camping, and other situations. Currently batteries are used for those man-portable situations. However, combustion engines could provide a better option based on their specific power and specific energy. Combustion engines have many different forms such as micro-turbines, micro-engines, Stirling cycles, and Organic Rankine Cycles. All must be considered when determining a system that can create a large amount of power and energy per unit of weight. External combustion engines may provide a great option because they can prevent losses and increase combustion efficiency, when compared with internal combustion engines. On the other hand, systems like Organic Rankine Cycles can operate with a lower temperature of the heat source.

Currently there is a market of internal combustion engines that are being made on a smaller scale known as Portable Generators. These portable fuel power generation systems are smaller than usual but are generally not quite small enough to meet the definition of man-portable. These systems were still studied to see if they meet the current needs of specific energy and specific power. Based on the Ragone Plots, the portable fuel power generation systems do not quite meet the standards of performance for what fuel power generation systems could meet for specific power. This in turn has kept the portable fuel power generation systems from being able to have a long enough time of autonomy to meet the needs for military use.

More research needs to be done on how to make man-portable fuel power generation systems more efficient and increase their specific power. This would allow for man-portable fuel power generation systems to be used in place of batteries for military operations. A change in the type of systems being used, such as external combustion engines, may provide the efficiency and specific power necessary to meet this demand.

\section{REFERENCES}

1. New York Independent System Operator, "Power Trends 2016 The Changing Energy Landscape," Mohawk, 2016.

2. CATRENE Working Group on Energy Autonomous Systems, "Energy Autonomous Systems: Future Trends in Devices, Technology, and Systems," CATRENE, 2009.

3. "Neutrium," 26 March 2014. [Online]. Available: https://neutrium.net/properties/specific-energy-and-energy-density-of-fuels/. [Accessed 5 October 2016].

4. U.S. Department of Energy, "Hydrogen Analysis Resource Center," January 2015. [Online]. Available: http:/ / bydrogen.pnl.gov/tools/ lower-and-bigher-heating-values-fuels.

5. R. Wilson, "The Energy Collective," 9 August 2013. [Online]. Available: http:/ / www.theenergycollective.com/ robertwilson190/257481/why-power-density-matters. [Accessed 5 October 2016].

6. Portable Generator Manufacturers' Association, PGMA, [Online]. Available: pgmaonline.com.

7. globalindustrial.com, "Yamaha EF2000iSv2 Portable Inverter Generator, 2000 Watt 79cc OHV 4-Stroke Gas CARB Compliant," Global Equipment Company Inc., 2016. [Online]. Available:

http:// wmw globalindustrial.com/p/ electrical/generators/portable/yamaha-ef2000isv2-portable-inverter-generator-2000-watt-carb-compliant. [Accessed 10 October 2016].

8. Department of Defense, "Department of Defense Dictionary of Military and Associated Terms," Suffolk, 2007. 
9. Mccloskey, Bryan D. "Expanding the Ragone Plot: Pushing the Limits of Energy Storage.” The Journal of Physical Chemistry Letters, vol. 6, no. 18, 2015, pp. 3592-3593., doi: 10.1021/acs.jpclett.5b01813

10. "Merriam-Webster Dictionary" (1828), Merriam-Webster. [Online]. Available: bttp:// wmw.merriam-webster.com/dictionary/portable.

11. K. S. J. D. James Jenden, "Energy Education," University of Calgary, [Online]. Available: bttp:// energyeducation.ca/encyclopedia/Energy_density_vs_power_density. [Accessed 18 October 2016].

12. National Academy of Sciences, "NCBI.gov," 2004. [Online]. Available: http://www.ncbi.nlm.nih.gov/books/NBK207797/.

13. Yunt, Mehmet, et al. "Designing Man-Portable Power Generation Systems for Varying Power Demand." AIChE Journal, vol. 54, no. 5, 2008, pp. 1254-1269., doi:10.1002/aic.11442

14. GoalZero, "Venture 30 Solar Recharging Kit," Goal Zero, 2016. [Online]. Available: bttp:// wmw.goalzero.com/p/317/venture-30solar-recharging-kit. [Accessed 10 October 2016].

15. Pulsar Products, inc., "Pulsar 1200W 2-Stroke Generator," Pulsar Products, inc., 2016. [Online]. Available: http://pulsarproducts.com/products/pg1202s/. [Accessed 10 October 2016].

16. GeneratorSmart, "GenSmart 1000," GeneratorSmart, 2016. [Online]. Available: http:/ / www.generatorsmart.com/ ?product=gensmart1000. [Accessed 10 October 2016].

17. Power Magazine, "Microturbine Technology Matures," Access Intelligence, Inc., 1 November 2010. [Online]. Available: http:/ / www.powermag.com/ microturbine-technology-matures/?pagenum=1. [Accessed 12 October 2016].

18. U.S. Department of Energy, "Combined Heat and Power Technology Fact Sheet Series," U.S. Department of Energy, 2016.

19. B. Sims, "Land 125 - Power Technologies Review," Land Operations Division, Edinburgh South Australia, 2012.

20. Organ, Allan J. "Stirling Cycle Engines: Inner Workings and Design," Hoboken: Wiley, 2013. EBooks on Demand.

21. Engineering Toolbox, "engineeringtoolbox.com," [Online]. Available: http:/ / www.engineeringtoolbox.com/adiabatic-flametemperature-d_996.html. [Accessed 16 November 2016].

22. Occupational Safety and Health Administration, "Fuel Handling and Storage." [Online]. Available: bttp:/ / www.osha.gov/pls/oshaweb/owadisp.show_document?p_table=STANDARDSOop_id=10420 [Accessed 15 April 2018]

23. National Academy of Sciences, "Meeting the Energy Needs of Future Warriors," National Academies Press, Washington, D.C., 2004.

24. J. Harper, "National Defense," NDIA, October 2015. [Online]. Available: bttp:/ / wmw.nationaldefensemagazine.org/archive/2015/October/Pages/TheArmyWantstoPowerUpDismountedSoldiers.aspx. [Accessed 11 December 2016].

25. A. W. Culp, Principles of Energy Conversion, Highstown: McGraw-Hill, 1991.

\section{ABOUT THE STUDENT AUTHOR}

Earl Allen obtained his B.S. in Mechanical Engineering in Spring 2017. He developed this research as part of his MENG 4395 Undergraduate Research course.

\section{PRESS SUMMARY}

Batteries are the most common source of portable electric power. However, thermally powered power generation systems offer high energy density and high power density making them candidates for further investigation as a feasible alternative. In this study, the feasibility of portable fueled power systems is investigated. 


\title{
The Role of Gender Stereotypes in Gubernatorial Campaign Coverage
}

\author{
Karen Bjerre \\ Department of Politics, Sewanee: The University of the South, Sewanee, TN \\ Student:bjerrkr0@sewanee.edu*,karen.bjerre@hotmail.com \\ Mentor:macrowde@sewanee.edu
}

\begin{abstract}
Through a content analysis of 1,152 articles covering 20 different gubernatorial races between 2004 and 2014, I examine how the presence of a woman candidate in a gubernatorial race affects media coverage of that campaign. This paper considers the prevalence of gender biases and stereotypes and examines whether print media outlets perpetuate these stereotypes or subvert them. Existing research suggests that voters favor traditionally masculine traits over traditionally feminine traits when evaluating candidates for public office. The difference in media coverage may therefore influence women's chances of getting elected, and thereby influence women's descriptive and substantive representation. I find that races with a woman candidate receive less issue coverage and more horse race coverage than races with two men running. Additionally, the types of adjectives used in campaign coverage differ depending on the gender of the candidate: women are more likely to be described as able to "change government," while men are more likely to be described as "experienced."
\end{abstract}

\section{KEYWORDS}

Media Coverage; Gender; Gubernatorial Campaigns; Women in Politics; Gender Stereotypes; Newspaper Coverage; American Politics

\section{INTRODUCTION}

At a campaign event about a month before the 2008 election, Beverly Perdue, a North Carolina gubernatorial candidate, shared her favorite introduction she had received with a crowd of supporters: "She's a good woman. But she fights like a man." 1 This statement exemplifies one of the many obstacles women face when running for elected office, in terms of gender stereotypes and social role congruity. The fact that being a "fighter" is inherently associated with masculinity forces women in political campaigns to pursue a delicate balance between being masculine enough for the job while maintaining enough femininity to be likeable. ${ }^{2}$ These gendered expectations are embedded in today's society and might explain why Claire McCaskill agreed to be interviewed in her kitchen, while running for Governor of Missouri. Being interviewed in her home emphasizes traditionally feminine characteristics, which could help balance out more assertive behavior in other contexts.

Even though women continue to be elected to political offices, the U.S. is still a long way from equal representation by gender. No level of office in the United States currently has more than 26 percent women representatives, and only six women are currently serving as governors of their states. ${ }^{3}$ For the time period examined in this paper $(2004-2014)$, eight of the ten races where a woman was competitive in the gubernatorial election, took place in states that had never had a woman governor. ${ }^{4}$ This underrepresentation is significant because it can affect substantive representation, future descriptive representation, and overall levels of participation for women. These patterns reinforce themselves in a cycle in which women have lower levels of political ambition than men, ${ }^{\mathbf{5}}$ they are more politically engaged when a woman is competitive in a given electoral contest, ${ }^{\mathbf{6}}$ and elected officials, who are women, are more likely to work on so called 'women's issues. ${ }^{7}$ Therefore, the descriptive representation of women at all levels of U.S. politics is important because it holds the potential to increase political ambition and efficacy among women and girls. ${ }^{6}$

In this paper, I examine how gender cues and stereotypes influence the media. Specifically, I test whether or not significant differences in news coverage exist between men and women candidates for governor. I choose to focus on gubernatorial contests because I am interested in the gender dynamics in elections for executive positions, and the gubernatorial level is the highest in which several women have both run and won. I find that the types of coverage each candidate receives differ by gender, especially when it comes to whether the coverage focuses on issue or horse race stories. Women candidates, as well as men running against women, are less likely than men running against men to receive substantive coverage of their issue positions.

I begin by establishing why it matters if women hold elected office. In that context, I address distinctions in issue competency evaluations, differences in legislative agendas developed by men and women, and the broader societal effects of electing women to office. To explain my focus on gubernatorial candidates, I then examine how theories of gender congruency and stereotypes affect women candidates and their constituents differently depending on the level of office in question. Finally, I look at how 
gender influences political campaigns in U.S. politics through the lens of media coverage, and how media coverage influences the broader public. This examination of existing scholarship lays the foundation for my hypotheses regarding how candidate gender affects media coverage.

\section{LITERATURE REVIEW}

Women as Elected Officials

I am evaluating how media coverage differs for men and women candidates to determine whether or not the media contributes to women's political underrepresentation. Some scholars have found effects of candidate gender on the issues covered in political campaigns to be negligible, and significantly smaller than party effects, ${ }^{8}$ while several other studies of politicians' legislative behavior indicate that the descriptive representation of women leads to more substantive representation of women. Specifically, when it comes to substantive representation, several scholars have found evidence that the legislative agendas for women differ from those of men, especially when it comes to working with "women's issues.", 9 These authors find that women senators are more likely to focus on issues such as education and health care in their legislative careers, while their colleagues prioritize issues surrounding the economy and national security. The findings suggest that gender might be a causal factor in the differences that develop between men and women in politics. However, the studies do not reveal why women pursue different legislative goals than men. Additionally, as these studies have focused on legislative positions at different levels, it is unclear how their findings translate to executive positions. Regardless of why these differences emerge, the descriptive representation of women appears to further the substantive representation of so called "women's issues."

These differences in legislative agendas align with classifications made by several researchers, to identify sets of policies as either masculine or feminine in nature. For example, Dolan identifies abortion, child care, education, and health care as traditionally feminine issues, while she categorizes the economy, national security, the deficit, immigration, and crime as masculine issues. ${ }^{10}$ These classifications are consistent with Fridkin and Kenney's ${ }^{9}$ framework for their analysis of senators' communications.

While a legislator might decide to work on specific issues on which he or she is particularly qualified, ${ }^{11}$ such decisions might also be made based on other factors such as perceived competency based on stereotypes, which can harm the prospects of women running for office. Several studies have found that issue competency evaluations from constituents vary both by partisanship and gender. ${ }^{12,13}$ This means that women are perceived as more competent at handling care issues, such as health care and education, while men seem to be particularly advantaged when it comes to perceptions of competence in the areas of national security. These differences function to create a sense of issue ownership based on gender that parallels the areas of issue ownership claimed by the Republican and Democratic party. ${ }^{14}$ These differences in competency evaluations seem likely to influence campaign and legislative activities alike, and therefore inform my expectations of how stereotypes might manifest themselves in campaign coverage, regarding both gender and partisanship.

Based on the distinctions of issue competencies, Kahn asserts that state offices might be more congruent with feminine stereotypes based on the increased focus on education and other social policies that are controlled at the state level. ${ }^{15}$ Ultimately, the differences in issue competency evaluations for men and women can be a hinderance for women running for office, considering Banwart's ${ }^{12}$ finding that stereotypically feminine issues are considered less important than issues such as the economy and national security. This leaves women politicians with a dilemma, since the issues they are considered the most competent at handling are also those generally rated as less important than the issues that favor their colleagues that are men. In the case of women running in gubernatorial races, these two approaches to stereotypes' role in politics point to different outcomes for women candidates. The first step must therefore be to establish whether these feminine stereotypes and issues are more prevalent in the women's gubernatorial campaigns.

This study seeks to enhance the knowledge of how gender stereotypes interact with media coverage, as recent research suggests that the dynamics of gender stereotypes on the campaign trail are changing. ${ }^{8,16}$ While research from the 1990 s and early 2000 s clearly indicated that gender stereotypes played a role in shaping how women candidates across different levels of office were perceived, ${ }^{17,18}$ more recent studies indicate that the prevalence of gender stereotypes, and their influence on women's electoral fortunes is declining. ${ }^{8,16}$ Additionally, Dolan and Lynch find that feminine stereotypes affect women candidates differently, depending on the candidate's partisanship. ${ }^{16}$ This study focuses on gubernatorial campaigns between 2004 and 2014 to explore the changing media landscape for women running for executive positions. This time frame is especially interesting, as it spans the apparent shift in how stereotypes affect the evaluations of women candidates, outlined above. ${ }^{8,16-18}$

Gender congruity

There are reasons to expect gender stereotypes to both help and hinder women gubernatorial candidates, depending on which stereotypes are primed by the media. The traditional lack of foreign policy or national security concerns associated with a governor may help women candidates overcome the barrier of traditional gender roles, which is one example of how traditional 
gender stereotypes sometimes counteract each other at higher levels of office. ${ }^{17}$ Eagly's and Karau's finding that "the activation of beliefs about women and men by gender-related cues... influences people to perceive women as communal but not very agentic and men as agentic but not very communal" (575), ${ }^{19}$ should help women candidates in the case of gubernatorial races, because many communal policy areas such as education child care are controlled at the state level. ${ }^{15}$ However, they also find that people are evaluated less positively when their social role is incongruent with their stereotype. This second aspect of their findings could negatively affect the evaluations of women running for office, especially offices with executive powers such as governors, which have traditionally been perceived as masculine in nature. ${ }^{18}$ Delineating the effects of stereotypes and gender role congruity is therefore complicated by the interactions between the two.

The case highlighted in the introduction exemplifies how women candidates might try to navigate this gendered terrain. Beverly Perdue's introduction: "She's a good woman. But she fights like a man"1 demonstrates a balance between being feminine enough to get the benefits of positive feminine stereotypes, while also projecting strength and assertiveness. Insofar as these frames translate into the media coverage, the candidate manages to combine the positive aspects of both masculine and feminine traits.

\section{Media effects}

Whether or not the media is receptive to the candidates' use or rejection of stereotypes matters to campaigns, as studies have shown that editorial slant and media coverage of campaigns influence the decisions of voters exposed to the coverage. ${ }^{20,21}$ Specifically, voters base their own evaluative parameters for candidates on the kinds of issues and traits local newspapers have been priming. ${ }^{20}$ Campaign coverage by newspapers influences issue salience, which in turn influences voters. ${ }^{21}$ This establishes an important baseline, since a study of newspaper coverage carries little weight if any effects found are unlikely to influence the public. However, based on these studies, I expect that any gender-based differences I identify in news coverage affect the parameters voters use to evaluate candidates.

Existing research on gender differences in campaign coverage of candidates is mixed in its findings. In their study of newspaper ideology and coverage of woman candidates, Shor et al.22 find that newspapers from across the ideological spectrum cover women candidates significantly less than men running for office. Contrasting these findings, Hayes and Lawless ${ }^{8}$ find that women congressional candidates do not face media coverage that differs from that of their men counterparts. Instead, they argue that partisanship is the driving force behind media coverage and voter behavior alike. An important aspect of this argument is that the novelty seems to have worn off for women congressional candidates, leading reporters to cover them similarly to men candidates. However, at the gubernatorial level, women candidates may still be a novelty (As of 2018, 39 women governors have served in 28 states), ${ }^{4}$ and the decreased novelty of women in legislative positions does not necessarily translate to executive positions, which makes gubernatorial races particularly interesting to study. Finally, as media attention increases with the level of office, the likelihood that gendered media coverage will affect voters can similarly increase.

Whether or not the media covers women candidates differently from their men counterparts is important because Fox and Lawless ${ }^{5}$ find that, in the development of political ambition, women's fear of facing biased media coverage presents a significant deterrent in the decision to run for elected office. This hesitation suggests that biased media coverage might be a contributing factor to the significant gender gap in descriptive representation. The differential media coverage based on gender does not influence candidate ambition alone. These differences matter as the media's coverage of women's campaigns for office exert a lot of power over how these women are perceived by the general public, especially if the news media perpetuates gendered stereotypes. ${ }^{15,18,23-25}$ These studies about gender cues in politics indicate that women candidates might not be able to escape gender stereotypes, even in the minds of the well-informed voter, since media priming has been shown to influence the criteria voters use to evaluate candidates. ${ }^{26}$ Differential coverage thereby becomes a problem insofar as the news media reinforces gender stereotypes, since this might make the negative effects of social role incongruity for women candidates even more severe. In other words, gendered media coverage may make it harder for women to win elected offices, depending on the stereotypes in question.

Even when women politicians seek to counter gender stereotypes about issue competency, the media is not necessarily responsive. In their comparison of candidates' communications and media coverage, Fridkin and Kenney find that, while women politicians often try to focus on masculine qualities on their own websites and in other candidate communications, these efforts are not reflected in the media coverage they receive. ${ }^{9}$ Instead, local newspapers seem to reinforce gender stereotypes in their coverage. This demonstrates that, while media coverage-at least in newspapers-rarely makes explicitly sexist comments, the gender-specific expectations of women candidates nevertheless influence how they are described and perceived.

Overall, the literature suggests that gender stereotypes not only affect candidates' and elected officials' behavior, they have also been known to influence the types of news coverage political candidates and issues receive, and how people evaluate peers and leaders. ${ }^{19}$ Media coverage, as the link between politicians and constituents, holds the potential to counteract stereotypes, but has not yet consistently done so, though recent research suggests that this tide is turning. ${ }^{8,}{ }^{16}$ Studying the extent of biased media 
coverage therefore provides us with an idea of how perceptions of politics and women candidates might be changed. Ultimately, while this project seeks to identify whether gender differences in newspaper coverage exists in gubernatorial campaigns, demonstrating the consequences of any such differences is beyond the scope of this study.

\section{Hypotheses}

I focus this study on gubernatorial races taking place between 2004 and 2014, as these races will generally both be high profile campaigns and be simultaneously incongruent with feminine stereotypes related to executive positions ${ }^{17,18}$ and congruent with stereotypes regarding feminine policy areas. ${ }^{15}$ These conditions are important to my research because they help ensure a wide enough set of data to allow me to draw meaningful conclusions about media coverage of women candidates, and because the consequences of media biases based on gender stereotypes seem especially complex at this level. Additionally, this time period covers the change in the literature outlined above, wherein gender stereotypes have moved from a central position in women's campaigns, ${ }^{17,18}$ to being far less prominent. ${ }^{16,21}$ As the more recent studies that find declining use of gender stereotypes all use data collected towards the end of my own dataset, ${ }^{16,21} \mathrm{I}$ primarily base my hypotheses on the earlier literature.

I examine differential media coverage through the lens of how gender trait descriptions and issue competency evaluations are used to describe candidates for elective office. ${ }^{12,13}$ These studies align with the distinctions made earlier about feminine versus masculine policy issues, where women are seen as more competent at dealing with the communal issues of health care and education, while men are seen as more competent at handling national security and the economy. Based on these considerations, I hypothesize the following:

Hypothesis 1: Men and women candidates will be more likely to receive newspaper coverage on issues that are congruent with their respective gender stereotypes.

Hypothesis 2: Men and women candidates will be more likely to be described using adjectives that are congruent with their respective gender stereotypes.

For gendered adjectives, I use Dolan's ${ }^{23}$ distinctions, which build on the work of Huddy and Terkildsen, ${ }^{13}$ between masculine traits, including being "decisive," "experienced," "intelligent," and a "strong leader" and feminine traits such as being "able to change government," “compassionate," "consensus building," and "honest." Similarly, I separate policy issues as well as gendered traits according to Dolan's ${ }^{23}$ work on gender stereotypes in politics. This research identifies abortion, child care, education, and health care as feminine issues, while the economy, national security, the deficit, immigration, and crime are categorized as masculine issues. These distinctions between masculine and feminine policy areas align with Eagly and Karau's ${ }^{19}$ framework on communal and agentic social roles. While theses stereotypes are rooted in binary ideas about gender, they represent views that are still broadly held by members of American society, ${ }^{19,27}$ and thus may have influence over the journalists who must produce coverage appealing to American media consumers. ${ }^{28}$ It is worth noting that these distinctions largely overlap with competency evaluations of the Democratic and Republican parties. ${ }^{14}$ For this reason, I control for the partisan affiliation of the candidates throughout my analysis.

In addition to news coverage that relies on gender stereotypes, women candidates are traditionally disadvantaged when it comes to receiving coverage of policy issues. Rather than issue coverage, some studies suggest they are more likely to be covered in terms of public opinion and campaign strategy. These horse race stories compose a large part of the coverage of political campaigns at all levels, but the problem seems to be especially salient for women candidates, ${ }^{9}$ which might be explained by the continued novelty of women candidates, especially at higher levels of office ${ }^{3}$ In their study of women candidates and the role of the women voters, Goldenberg and $\mathrm{Kahn}^{29}$ found that women candidates not only get less coverage, but the coverage they do receive is more likely to focus on horse race stories than that of their men colleagues, meaning that news stories tend to focus more on a candidate's viability, endorsements, or campaign funds than his or her policy positions. Based on these findings, I hypothesize that:

Hypothesis 3: Horse race coverage will constitute a larger proportion of women candidates' news coverage than the corresponding proportion for men candidates. It follows that issue coverage will constitute a smaller proportion of women candidates' news coverage than the corresponding proportion for men candidates.

A lack of issue coverage poses a challenge to the women candidates, as issue-oriented news coverage can help voters formulate criteria on which to judge candidates. ${ }^{20,21}$ Less issue coverage might therefore lead voters to be more reliant on gender cues and stereotypes in their voting decision than might otherwise be the case or cause them to think women candidates lack issue competency. 
Finally, I am interested in whether differential coverage applies only to women candidates, or if the effects of gender stereotypes influence the media's coverage of the men running against them as well. Addressing how men running against women are covered differently than men running against other men might indicate the direction in which campaign coverage is headed, as women candidates for executive offices become more common. While I am not aware of any research that addresses how campaign coverage differs for men running against women as opposed to coverage of campaigns with two men running against each other, I do expect these men to be covered differently, as the presence of a women candidate makes gender more salient to reporters. This expectation leads me to hypothesize that:

Hypothesis 4: Mixed-gender* campaigns will be covered differently on each of the distinctions outlined in the previous hypotheses than campaigns in which two men are running against each other.

Ideally, I would also examine how coverage differs for women depending on the gender of their opponent; however, there is not enough available data for competitive gubernatorial races with two women running against each other.

\section{METHODOLOGY}

In order to test my hypotheses, I conducted a content analysis of news articles covering 20 different competitive, open seat gubernatorial campaigns. Print media, specifically newspapers, has been shown to be more effective at transmitting political information to consumers than TV and radio news, ${ }^{30}$ and has been shown to influence the criteria voters use to judge political candidates. ${ }^{26}$ Newspapers are expected to have a relatively higher standard of unbiased writing than TV sources, ${ }^{30,31}$ and are also more likely to frame their campaign coverage as issue stories. ${ }^{26}$ Therefore, if I find evidence of gender differences in media coverage among newspaper stories, this is likely a conservative estimate of bias compared to what is present in online or television news sources. I focus on open seat races to address Arnold's conclusion that newspapers cover incumbents and challengers differently. ${ }^{33}$ For the purposes of this project, I define competitive races by the standard established by Atkeson as contests with "a $10 \%$ or lower margin of victory between the winner and the runner up" (p. 1046). ${ }^{6}$

I establish a matched pair design by pairing competitive races with at least one woman candidate (one contest, New Mexico 2010 , had to women candidates) with similar races with only men candidates. The list of the 20 pairs (40 candidates total) I study can be found in Table 1 of the appendix. When pairing races with similar contests, I considered geographical region, year, and demographic similarity on factors such as state partisanship and ethnic composition. The matched pair design helps account for underlying factors that might otherwise confound my findings, as the pairs serve to essentially control for differences based on demographic or temporal effects. Pairs are made within the same presidential election cycle to ensure similarity in the national political context.

To find articles about my selected candidates, I searched Lexis Nexis, a database containing content from more than 3,000 newspapers. In my search on Lexis Nexis, I looked for articles that mentioned the candidate's name three months prior to the election. Using a random number generator, I selected 30 articles per candidate, except in cases where fewer than 30 articles were available (Brian Schweitzer, MT 2004; Bob Brown, MT 2004; Dina Titus, NV 2006), for a total of 1,152 observations. I coded each article on an array of variables including mentions of a candidate's appearance, whether the story was horse race coverage or issue focused, the general tone of coverage (positive, neutral, or negative), and mentions of specific issues (full list in the appendix). The policy issues for which I coded were classified as either masculine or feminine. The measure of inter-coder reliability is outlined in the appendix along with the codebook.

To define masculine and feminine policies, I followed the model used by Dolan in chapter three "When Does Gender Matter?"10 Her list of feminine policies includes abortion, child care, education, and health care, while her list of masculine policies includes crime, the deficit, the economy, immigration, and national security. This model is similar to those used by other scholars. ${ }^{3}$ Similarly, I used Dolan's distinctions between masculine traits (decisive, experienced, intelligent, strong leader) and feminine traits (able to change government, compassionate, consensus building, honest). ${ }^{10}$

I created scales for policy mentions by combining all the masculine policies into one dependent variable and all the feminine policies into another dependent variable. These scaled variables add up the number of mentions of each policy issue, with possible values for each article ranging from zero (no mentions of any of the policies) to five (mentions all the individual policies). I used the same structure in setting up scales for masculine and feminine trait mentions. Finally, I established another dependent variable

\footnotetext{
${ }^{*}$ While it is important to note that gender is not a simple binary identity, in this project I am constrained to studying the candidates that have sought gubernatorial office in the United States so far, all of whom have presented themselves in ways consistent with a male or female gender identity. Therefore, I have developed hypotheses and analyses organized around this gender identity comparison, and I look forward to future work which can investigate media portrayals of candidates who identify differently regarding gender in the future.
} 
by coding every article as focused on either issue- or horse race coverage. I classified stories as issue oriented if their focus was on specific policy positions, or challenges facing the state, while stories were classified as horse race coverage if they focused on polls, campaign events, fundraising efforts or endorsements.

In order to assess the overarching frames used in the campaign coverage of women candidates, I recorded all categories of data based on mentions of relevant words and phrases regardless of whether the word or trait was ascribed to the candidate in question or a related person in the article. I base this decision on the idea that being compared to a woman candidate might lead men candidates to be measured by traits that I categorize as 'feminine,' such as honesty and consensus building. Likewise, I record instances where a candidate is described as lacking in one of these qualities in the same way because these instances still convey the importance of said trait to the evaluation of the candidate. In addition to evaluating overarching frames of the campaigns, this decision also helped reduce discrepancies between coders, thereby strengthening the inter-coder reliability.

Based on several studies that have found that issue competency evaluations differ by both gender and partisanship, ${ }^{12-14}$ and that issue competency, especially concerning national security, is evaluated differently for Republican and Democratic women, ${ }^{27} \mathrm{I}$ included a control variable for party ID throughout my analysis. I also controlled for presidential election cycle, coded as either pre- or post-2008, to account for the potentially confounding factor of presidential agenda setting. Specifically, health care, an issue classified as feminine, received prominent coverage in the post-2008 period based on presidential politics rather than gender. Similarly, the economic downturn in 2008, and subsequent efforts to stimulate the economy, may have led to an increase in coverage of economic issues post-2008 for reasons unrelated to gubernatorial candidate gender. While this dummy-variable is unlikely to capture all the effects, it is captures the major trends related to presidential politics.

\section{DATA AND RESULTS}

The first two columns of Table 1 show the results of a regression analysis with each of the policy scales as the dependent variable, containing the policy issues discussed in the methodology section, and includes controls for presidential cycle (pre-2008) and partisanship. The coefficient for the gender variable for the feminine policy scale is negative and marginally statistically significant $(\mathrm{p}<0.1)$, which indicates that women candidates are less likely than their men counterparts to receive coverage on issues considered traditionally feminine. This means that my first hypothesis is not supported for women candidates; rather than receiving more coverage on feminine issues, women running for office receive significantly less coverage on these issues than their men counterparts. For the other dependent variable, the masculine policy scale, the gender coefficient does not reach statistical significance. The analysis of the combined policy scales therefore does not support my first hypothesis.

\begin{tabular}{|c|c|c|c|c|c|}
\hline & $\begin{array}{c}\text { Feminine Policy } \\
\text { Scale }\end{array}$ & $\begin{array}{c}\text { Masculine Policy } \\
\text { Scale }\end{array}$ & $\begin{array}{l}\text { Feminine Trait } \\
\text { Scale }\end{array}$ & $\begin{array}{c}\text { Masculine Trait } \\
\text { Scale }\end{array}$ & Issue Coverage \\
\hline Woman & $\begin{array}{c}-0.0827 \dagger \\
(0.050)\end{array}$ & $\begin{array}{c}-0.0214 \\
(0.455)\end{array}$ & $\begin{array}{c}0.0642 * * \\
(0.021)\end{array}$ & $\begin{array}{c}-0.0392 * \\
(0.019)\end{array}$ & $\begin{array}{c}-0.0531 \\
(0.032)\end{array}$ \\
\hline Democrat & $\begin{array}{l}-0.0005 \\
(0.045)\end{array}$ & $\begin{array}{l}-0.0281 \\
(0.042)\end{array}$ & $\begin{array}{l}-0.0224 \\
(0.015)\end{array}$ & $\begin{array}{l}-0.0164 \\
(0.020)\end{array}$ & $\begin{array}{c}0.04670 \\
(0.029)\end{array}$ \\
\hline Pre-2008 & $\begin{array}{l}0.0165 \\
(0.045)\end{array}$ & $\begin{array}{l}-0.0608 \\
(0.041)\end{array}$ & $\begin{array}{l}0.0215 \\
(0.014)\end{array}$ & $\begin{array}{l}-0.0059 \\
(0.020)\end{array}$ & $\begin{array}{c}0.01039 \\
(0.029)\end{array}$ \\
\hline Constant & $\begin{array}{c}0.4780 * * \\
(0.038)\end{array}$ & $\begin{array}{c}0.4694 * * \\
(0.035)\end{array}$ & $\begin{array}{c}0.0317 * * \\
(0.010)\end{array}$ & $\begin{array}{c}0.1192 * * \\
(0.019)\end{array}$ & $\begin{array}{c}0.29401 * * \\
(0.024)\end{array}$ \\
\hline
\end{tabular}

Table 1. Policy and Trait Coverage of Women Candidates-Table shows OLS regression coefficients with standard errors in parentheses. ${ }^{t} \mathrm{p}<0.1 \quad *_{\mathrm{p}}<0.05 \quad * *_{\mathrm{p}}<0.01$

When evaluating coverage of the individual issues contained in the policy scales, it becomes clear that gender does not influence coverage in the same way for each issue. The coefficients and confidence intervals for the Woman independent variable in individual regressions with media coverage of each policy area as a dependent variable are graphed in Figure 1. The gender variable fails to reach statistical significance for several of the policy issues. Education, crime, the economy, the deficit, and immigration are all issues where the gender variable is not statistically significant. However, counter to my hypothesis, the gender coefficient for coverage of both health care and abortion is negative and statistically significant $(p<0.05)$. This indicates that men candidates are more likely than women candidates to receive coverage of these issues otherwise classified as feminine. On the other hand, the child care and security variables both act as expected, with a gender coefficient that is positive and statistically 
significant $(\mathrm{p}<0.05)$ for child care, and a negative and statistically significant coefficient for security ( $<<0.05)$. So, while these two variables support my initial hypothesis, the results from the other policy issues are inconsistent with my expectations.

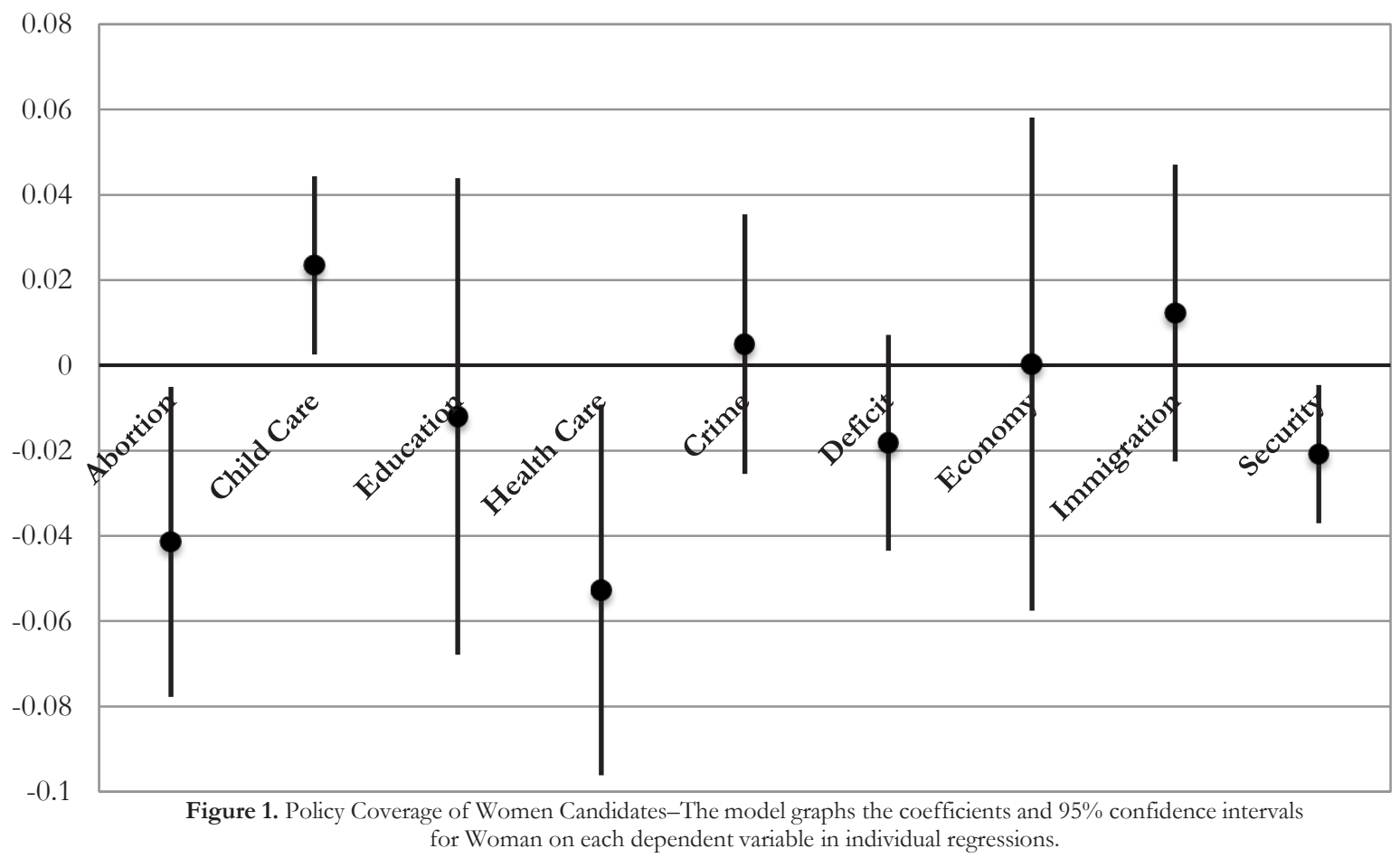

For my second hypothesis, that candidates will be more likely to be described using adjectives that are congruent with gender stereotypes, I develop a similar system. I created masculine and feminine trait scales, with possible values between zero (no mentions of any of the traits) to five (mentions all the individual traits), based on Dolan's ${ }^{10}$ framework. The third and fourth columns of Table 1 show the results of regression analyses on masculine and feminine trait scales, with a measure of candidate gender as the key independent variable and controls added for temporal effects and party ID. The results are consistent with my hypothesis, with a positive and statistically significant $(\mathrm{p}<0.05)$ coefficient for gender on the feminine trait scale, and a negative and statistically significant $(\mathrm{p}<0.05)$ coefficient for gender on the masculine trait scale. Together, these results support my second hypothesis; they confirm that women candidates are overall more likely to be described using traditionally feminine adjectives, while men candidates are more likely to be described using traditionally masculine adjectives.

Figure 2 shows the coefficients and confidence intervals of individual regressions with the media coverage of individual traits as the dependent variables and with gender as the key independent variable. The graph shows that being described as being able to 'change government' is individually significant $(\mathrm{p}<0.05)$ with a positive coefficient that supports my hypothesis. While not immediately obvious, since the figure graphs a $95 \%$ confidence interval, the honesty and experience variables both reach statistical significance with the gender variable at $\mathrm{p}<0.1$ and $\mathrm{p}<0.06$ respectively. Both of these dependent variables also act in the expected direction, which further supports my hypothesis. While several of the coefficients for Woman in these individual models do not reach statistical significance, the results suggest that women candidates are more likely to be described as honest and able to 'change government,' while men candidates are more likely to be described as experienced. Finally, the "decisive" trait previously identified as masculine was not used as a descriptor in any of the articles in the dataset and was therefore dropped from the analysis. 


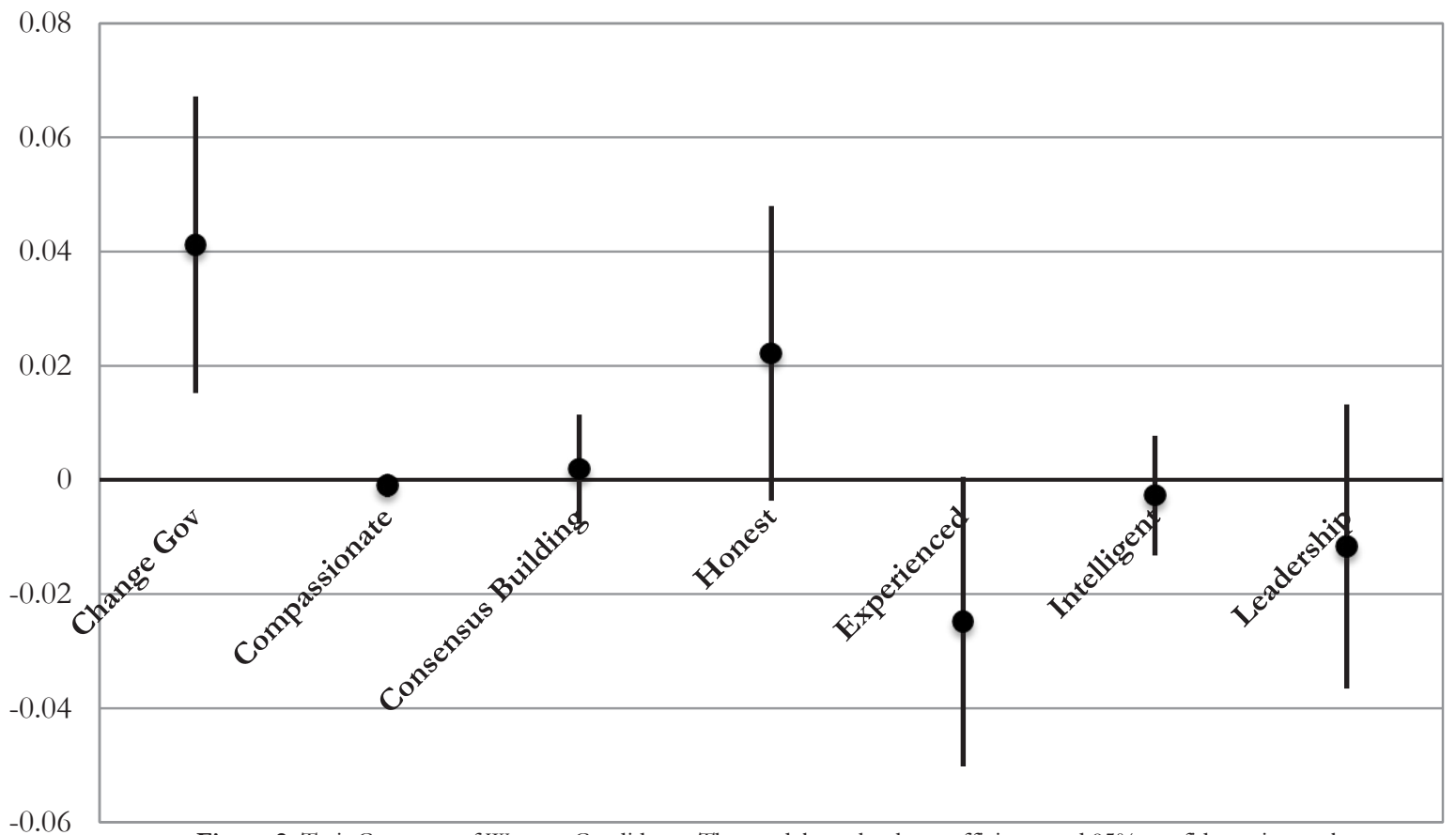

Figure 2. Trait Coverage of Women Candidates-The model graphs the coefficients and 95\% confidence intervals for Woman on each dependent variable in individual regressions.

For my third hypothesis regarding issue coverage versus horse race stories, I start by examining the percentage of horse race stories by gender. I find that about six percentage points more of the coverage of women candidates is classified as horse race stories than the corresponding coverage level for men candidates. However, as seen in the fifth column of Table 1, the regression analysis shows that these results fall just short of statistical significance $(p=0.101)$. However, as the first column of Table 2 shows, a similar analysis of mixed-gender races indicates that these receive significantly less issue coverage than races in which two men are running against each other. While my hypothesis is not directly supported, I do find evidence that the presence of a woman candidate leads to a lower level of issue coverage for both her and her opponent. This trend might be explained by a focus on the gender frame rather than a more traditional frame. For example, stories about potentially electing the state's first woman governor were prevalent in races with a woman candidate, but obviously not so in races with two men candidates.

\begin{tabular}{|c|c|c|c|c|}
\hline & Issue Coverage & $\begin{array}{c}\text { Feminine Policy } \\
\text { Scale }\end{array}$ & $\begin{array}{c}\text { Masculine Policy } \\
\text { Scale }\end{array}$ & Issue Coverage \\
\hline Mixed-gender & $\begin{array}{c}-0.0976^{* *} \\
(0.028) \\
\end{array}$ & & & \\
\hline $\begin{array}{c}\text { Man w/Woman } \\
\text { Opponent }\end{array}$ & 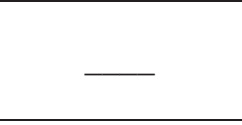 & $\begin{array}{c}-0.2801 * * \\
(0.051) \\
\end{array}$ & $\begin{array}{c}-0.0908 \dagger \\
(0.051) \\
\end{array}$ & $\begin{array}{c}-0.1099 * * \\
(0.035) \\
\end{array}$ \\
\hline Democrat & $\begin{array}{l}0.0319 \\
(0.028) \\
\end{array}$ & $\begin{array}{c}-0.0883^{\dagger} \\
(0.053) \\
\end{array}$ & $\begin{array}{r}-0.0601 \\
(0.049) \\
\end{array}$ & $\begin{array}{l}0.0345 \\
(0.035)\end{array}$ \\
\hline Pre-2008 & $\begin{array}{l}0.0086 \\
(0.028) \\
\end{array}$ & $\begin{array}{l}0.0481 \\
(0.051) \\
\end{array}$ & $\begin{array}{l}0.0104 \\
(0.049)\end{array}$ & $\begin{array}{c}-0.0122 \\
(0.033) \\
\end{array}$ \\
\hline Constant & $\begin{array}{c}0.3377^{* *} \\
(0.028)\end{array}$ & $\begin{array}{c}0.5931 * * \\
(0.049)\end{array}$ & $\begin{array}{c}0.4810^{* *} \\
(0.042)\end{array}$ & $\begin{array}{c}0.3456^{* *} \\
(0.031)\end{array}$ \\
\hline
\end{tabular}

Table 2. Policy and Trait Coverage of mixed-gender contests and men candidates with women opponents-Table shows OLS regression coefficients with standard errors in parentheses. ${ }^{\dagger} \mathrm{p}<0.1 \quad * \mathrm{p}<0.05 \quad * *_{\mathrm{p}}<0.01$ 
The analyses so far have evaluated simply if women candidates are covered differently from men candidates, regardless of whether the men candidates were running against a woman or another man. However, it is possible that men facing women opponents may be covered differently than those facing men opponents. To test if this is the case, I examine my fourth hypothesis, that mixed-gender campaigns will be covered differently than campaigns where two men are running against each other. I run analyses similar to those above, but with a different gender-related dummy variable. This variable is coded as 1 if the race is mixed gender, and 0 if both candidates are men. The results in Figure 3 are similar to those evaluating women versus men candidates' coverage. Abortion, education, and health care all have gender coefficients that are negative and statistically significant ( $p<0.01)$, which indicates that candidates in mixed-gender races receive less coverage of these issues than candidates in men-only races. For masculine issues, coverage of the deficit reaches statistical significance $(p<0.01)$ with a negative coefficient. None of the other policy variables reach statistical significance in this model, indicating that, for masculine policy issues, candidates in mixed-gender races are only disadvantaged on issue coverage of the deficit.

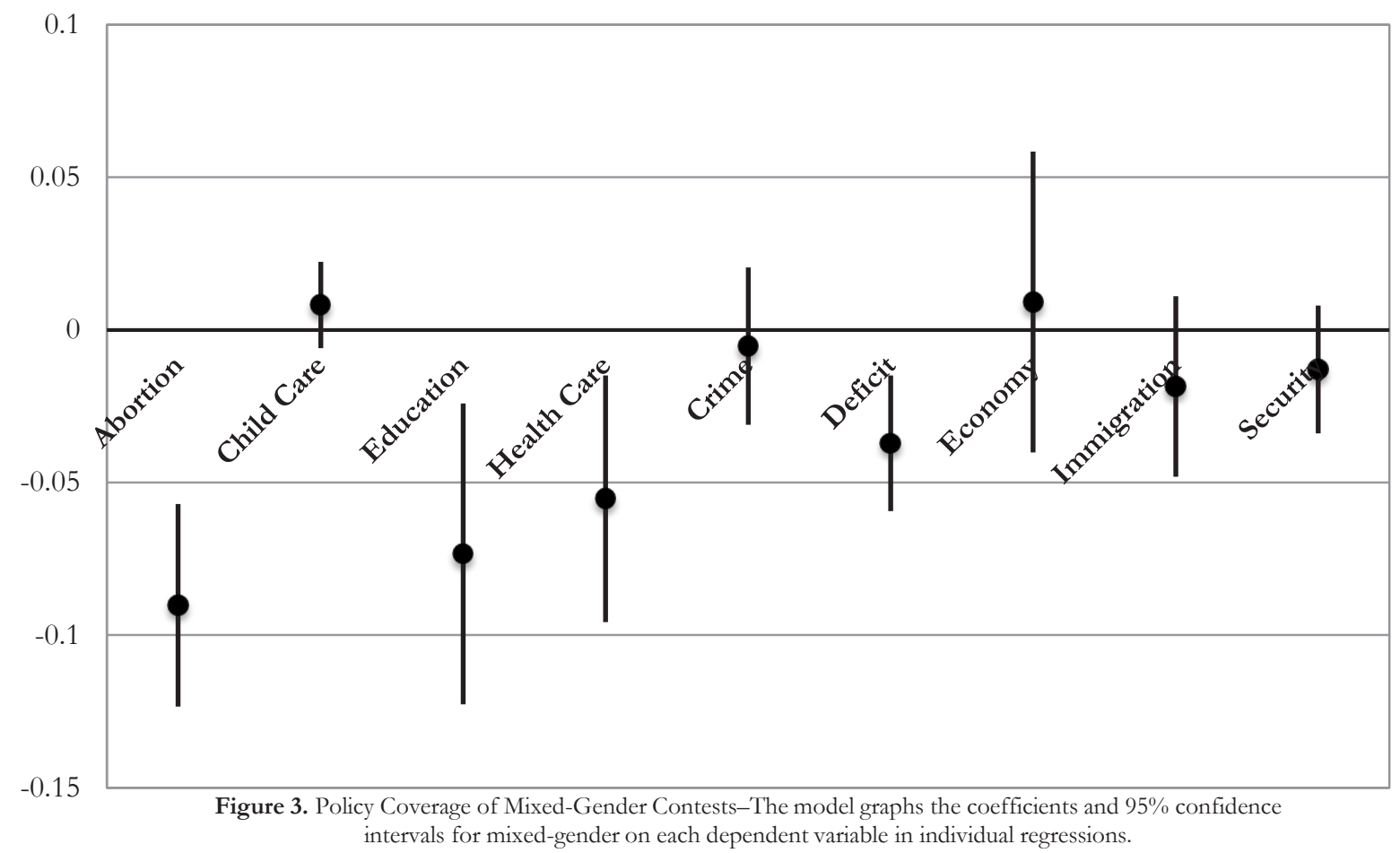

In an attempt to delineate whether the differences in coverage of mixed-gender races are due to coverage specifically of the men or women candidates, I examine gender in a third context: I limit my analysis to only the men candidates, and test how men running against women are covered differently from men running against men. Table $\mathbf{5}$ shows the results of the regression analysis of the masculine and feminine policy scale variables. In this case, the gender coefficient for both dependent variables is negative and statistically significant $(\mathrm{p}<0.1$ and $\mathrm{p}<0.001$ respectively), which indicates that men running against women are less likely than men running against men to receive coverage on both masculine and feminine policy issues.

Figure 4 shows the results from individual regressions with each specific policy issue as the dependent variables and with opponent gender as the independent variable. This analysis comes out with statistically significant coefficients for five of the nine policy issues. Significantly, all of these coefficients are negative, indicating that men running against women are overall less likely to receive issue coverage than men running against men. To confirm this result, I run a final regression analysis for the effect of opponent gender on issue versus horse race coverage, the results of which are in Table 6.

As expected, the coefficient for opponent gender is negative and statistically significant $(\mathrm{p}<0.01)$, supporting my assertion that men running against women receive significantly less issue coverage than men running against men. This final measure also supports my hypothesis that mixed-gender campaigns are covered differently from men-only races. 


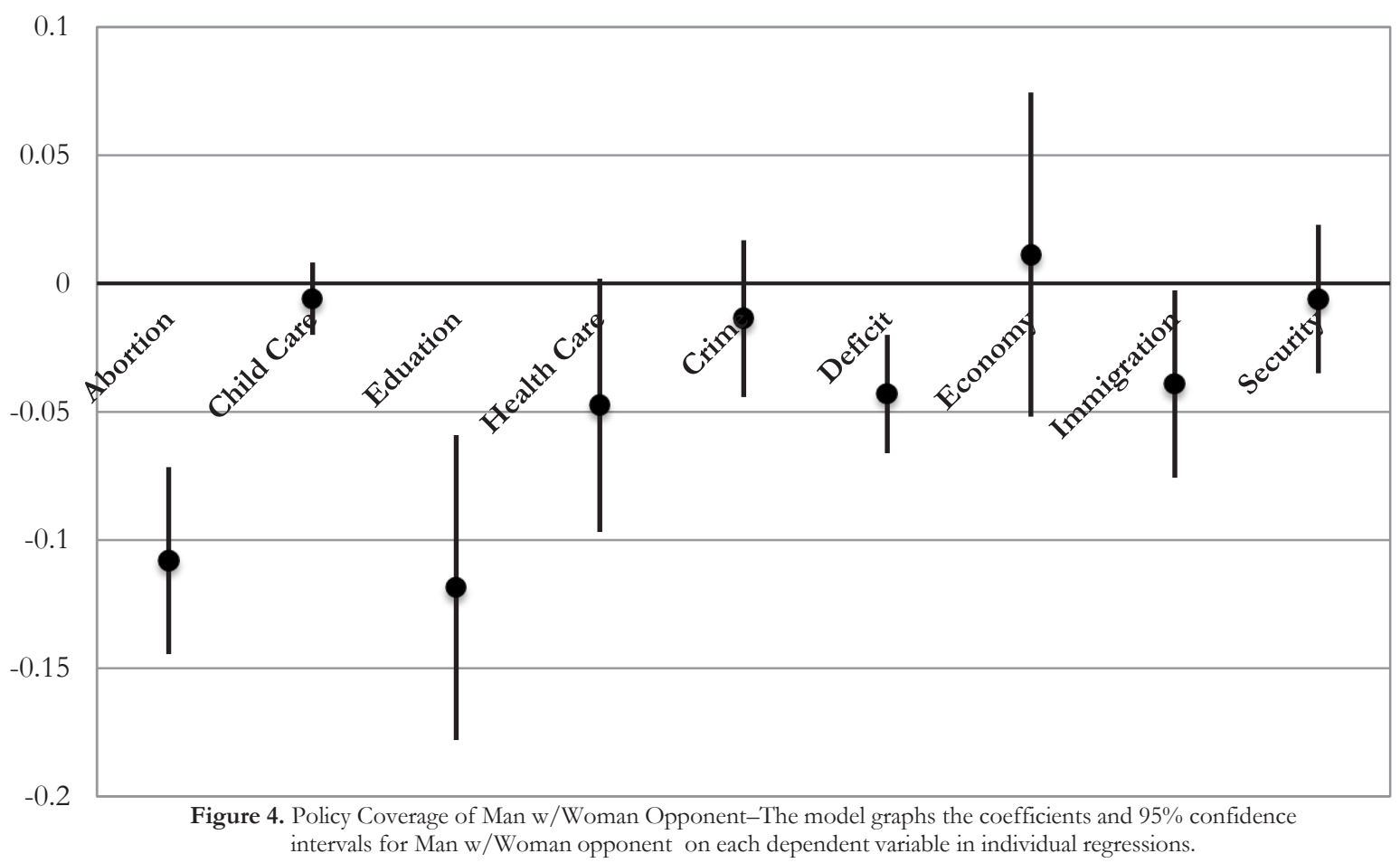

\section{DISCUSSION}

The findings of this paper clearly indicate that differences in media coverage exist depending on the gender of the candidates in gubernatorial elections. Although my findings provide mixed support for my first hypothesis, there is evidence of significant differences in newspaper coverage of men and women candidates. In terms of issue coverage, women candidates are more likely than men candidates to receive coverage on the issue of child care, but less likely to receive coverage on abortion, health care, and security issues. This is especially significant because several of the issues on which women receive less coverage are those where previous research suggests they would be advantaged by gender stereotypes. ${ }^{12,13,15,16}$ This suggests that gender stereotypes do not lead to increased coverage of the relevant issues in the newspaper coverage of gubernatorial campaigns. On the other hand, the difference might be a manifestation of Dittmar's finding that "male candidates, especially Democrats for whom women are a base of support, utilize gender-based messages and messengers to compensate for women's perceived natural advantage with female voters in mixed-gender contests". 35 This quotation suggests that the media's focus on some stereotypes over others may be a response the candidates' messaging rather than simple stereotyping. However, this explanation does not align with my findings that men running against women receive significantly less issue coverage on traditionally feminine issues than men running against men, unless men candidates in mixed-gender races discuss issues less. Finally, the reasons behind stereotyped coverage neither increase nor decrease the effects of gendered frames.

When it comes to adjectives used to describe candidates, I find evidence that the words used by journalists align with gender stereotypes. With women candidates especially likely to be described as able to "change government," and men candidates described as "experienced," campaign coverage reinforces gender stereotypes. While being able to "change government" can be considered a good quality, especially in anti-incumbent elections, the priming of gender overall seems likely to hurt rather than help women candidates for executive offices. ${ }^{18,36}$ Additionally, Dolan and Lynch, in a recent study of gender stereotypes in a range of electoral contexts, find that only a limited number of stereotyped traits are used by voters. ${ }^{16}$ Interestingly, while they measure all of the same stereotypes as this study, the ones that emerge in as significant in their study are those of women as more compassionate and men as more decisive ${ }^{16}$ - a different set of traits than those that emerged in this study. This difference might be explained by temporal changes reflected in the analysis, as their data focuses on a single election cycle (2010) rather than campaigns spread out over several years. Finally, while this study focuses on newspaper coverage, Dolan and Lynch's research surveys voters directly. The variability between the results might therefore stem from differences in how newspapers and voters treat stereotypes, or an effect of some confounding variables that were unaccounted for. 
Another aspect of newspaper coverage which can significantly influence voters, and thereby candidates' success, is issue coverage. ${ }^{20,21}$ While the differences I find in levels of issue coverage for women candidates fall just short of statistical significance, my analysis of mixed-gender versus all-men races indicates that there are differences that go beyond women candidates. These findings demonstrate that the effects of having a woman run for governor are not limited to the woman's campaign. Instead, the races overall receive less issue coverage than a race with two men candidates. If this trend is a product of the novelty factor that still seems to be associated with women gubernatorial candidates, the levels of issue coverage is likely to rebound to the same level as that of the races with two men running. However, if the trend continues as the novelty of women gubernatorial candidates wears off, this could mean an overall lower standard for coverage of policy positions in the future, if more women run for office.

Having candidates receive less issue coverage can potentially influence voter information levels about the candidates, ${ }^{26}$ and thereby cause voters to rely more heavily on gender cues. ${ }^{37}$ One explanation for the differences in levels of issue coverage might be that newspapers are more likely to cover an election with a woman candidate by focusing on gender and the potential for electing a woman governor for the first time. This frame seems to lead to more focus on the candidate's viability, and thereby more horse race coverage. However, as long as both the man and woman in the same race receive similar amounts of issue coverage, as the data suggests, the detrimental effects of the gender frame would be limited to those associated with priming gender prior to an election for an executive office..$^{18,36}$

The impact of a woman candidate on the campaign coverage is, surprisingly, the most obvious when comparing the coverage of men candidates with women opponents to men candidates with men opponents. The levels of issue coverage for men in mixedgender races are significantly lower than the corresponding levels for men in men-only contests. This difference is highlighted as nearly half of the dependent policy variables from masculine and feminine policy areas alike indicate that men running against women receive less coverage on those policy issues than their colleagues with men opponents. The lower levels of issue coverage might be explained by the media's tendency to cover mixed-gender races in terms of gendered frames, as discussed above.

This finding is perhaps the most interesting, as mixed-gender races are likely to become more common over time. However, as mixed-gender races become more common, and the novelty of having a woman run for governor wears off, we might see more similar news coverage between different types of contests. ${ }^{8}$ Additionally, Eagly and Karau assert that "to the extent that leader roles become more androgynous, the tendency to perceive women as less qualified than men should lessen" (578). ${ }^{19}$ This is another factor that could influence future campaign coverage, as a change in perception where leadership roles are seen as more androgynous could cause gender priming to be less powerful in candidate coverage and evaluations. However, considering that women's levels of representation across levels of office are increasing at low rates, and that women represent less than 30 percent of elected officials at all levels of office, ${ }^{3}$ this move towards more androgynous perceptions of leadership roles seems unlikely to happen any time soon.

Overall, the data shows limited evidence of gender stereotypes being used in newspaper coverage of gubernatorial campaigns, while most variables fail to reach statistical significance. The implications of these findings are that, while some gender stereotypes still influence aspects of gubernatorial campaigns, the assertions of recent studies, that the prevalence of gender stereotypes is in decline, ${ }^{8,16}$ seem plausible. With a decreasing emphasis on gender stereotypes, more women might pursue elected office, if the barrier of a perceived hostile media environment is reduced. ${ }^{5}$ As outlined earlier, electing more women to office could thereby improve women's descriptive as well as substantive representation.

To further address gendered differences in news coverage, future research should focus on how races with men running against women differ from races with two women candidates. One problem with this line of research is the limited amount of data available, since these contests are still fairly rare, especially at the statewide level. Media coverage from outlets other than newspapers might also provide different frames for mixed-gender races. The perpetuation of gender stereotypes through cable news, social media, and political blogs are all potential areas that could complement this study. This aspect requires further research, especially considering the expanding knowledge gap and difference in information sources between the most and least informed voters. ${ }^{38}$ Finally, as more women are successful in their bids for state executive positions, new possibilities emerge with regards to studying the role of incumbency for women governors, as well as changes in the media frames, as the novelty of women candidates for executive positions wears off.

\section{ACKNOWLEDGMENTS}

I am thankful to the Politics department at Sewanee: The University of the South and the Dugan Education and Research Grant for support throughout the research process, the anonymous reviewers for their feedback, and Drs. Melody Crowder-Meyer and JoyAnna Hopper for their guidance and help from start to finish. 


\section{REFERENCES}

1. Mayo, N. (2008) Perdue Pledges Not to Forget Small Counties. Sun Journal.

2. Williams, J., Dempsey, R. (2014) What works for women at work: four patterns working women need to know, NYU Press, New York.

3. CAWP. Women in Elective Office 2018. bttp:// wnw.cawp.rutgers.edu/women-elective-office-2018 (accessed February 4th, 2018).

4. CAWP. History of Women Governors. http:/ / www.cawp.rutgers.edu/ history-women-governors (accessed February 9 $9^{\text {th }}, 2018$ ).

5. Fox, R. L., Lawless, J. L. (2014) Uncovering the Origins of the Gender Gap in Political Ambition. American Political Science Review 108(3), 499-519.

6. Atkeson, L. R. (2003) Not All Cues Are Created Equal: The Conditional Impact of Female Candidates on Political Engagement The Journal of Politics 65(4), 1040-1061.

7. Swers, M. L. (2013) Women in the Club: gender and policy making in the senate. The University of Chicago Press, Chicago.

8. Hayes, D., Lawless, J. L. (2016) Women on the Run: Gender, Media, and Political Campaigns in a Polarized Era. Cambridge University Press, New York.

9. Fridkin, K., Kenney, P. (2013) The Changing Face of Representation: The Gender of U.S. Senators and Constituent Communications, University of Michigan Press, Ann Arbor, MI.

10. Dolan, K. (2014) Attitudes, Stereotypes, and Support for Women Candidates, in When Does Gender Matter? 49-90. Oxford University Press, New York.

11. Volden, C., Wiseman, A. E. (2014) The Habits of Highly Effective Lawmakers, in The Lawmakers: Legislative Effectiveness in the United States Congress, Cambridge University Press, New York.

12. Banwart, M. (2010.) Gender and Candidate Communication: Effects of Stereotypes in the 2008 Election. American Behavioral Scientist 54(3), 265-283.

13. Huddy, L., Terkildsen, N. (1993) Gender stereotypes and the perception of male and female candidates. American Journal of Political Science 37(1), 119-147.

14. Benoit, W., Hansen, G. (2004) Issue ownership in primary and general presidential debates. Argumentation and Advocacy 40(3),143-153.

15. Kahn, K. (1994) Does Gender Make a Difference? An Experimental Examination of Sex Stereotypes and Press Patterns in Statewide Campaigns. American Journal of Political Science 38(1), 162-195.

16. Dolan, K., Lynch, T. (2016) The Impact of Gender Stereotypes on Voting for Women Candidates by Level and Type of Office, in Politics \& Gender 12, 573-595.

17. Dolan, K. (1997) Gender Differences in Support for Women Candidates. Women \& Politics 17(2), 27-4.

18. Fox, R. L., Oxley, Z. M. (2003) Gender stereotyping in state executive elections: Candidate selection and success. Journal of Politics 65(3), 833-850.

19. Eagly, A. H., Karau, S. (2002) Role congruity theory of prejudice toward female leaders. Psychological Review 109(3), 573-598.

20. Druckman, J. N., Parkin M. (2005) The Impact of Media Bias: How Editorial Slant Affects Voters. Journal of Politics 67(4), 1030-1049.

21. Hayes, D. (2008) Does the Messenger Matter? Candidate-Media Convergence and Its Effects on Voter Issue Salience. Political Research Quarterly 61(1), 134-146.

22. Shor, E., Rijt, A., Ward, C., Askar, S., Skiena, S. (2014) Is There a Political Bias? A Computational Analysis of Female Subjects' Coverage in Liberal and Conservative Newspapers. Social Science Quarterly 95(5) 1213-1229.

23. Dolan, K., Deckman, M., Swers, M. (2011) Women as Candidates for Elective Office, in Women and Politics 128-171, Pearson, New York.

24. Fowler, L., Lawless, J. L. (2009) Looking for Sex in All the Wrong Places: Press Coverage and the Electoral Fortunes of Gubernatorial Candidates. Perspectives on Politics 7(3), 519-536.

25. Herrnson, P. S., Lay, C., Stokes, A. K. (2003) Women Running 'as Women': Candidate Gender, Campaign Issues, and VoterTargeting Strategies. The Journal of Politics 65(1), 244-255.

26. Druckman, J. N. (2004) Priming the Vote: Campaign Effects in a U.S. Senate Election. Political Psychology 25(4), 577-594.

27. Koenig, A. M., Eagly, A. H., Mitchell, A. A., Ristikari, T. (2011) Are leader stereotypes masculine? A meta-analysis of three research paradigms. Psychological Bulletin 137(4), 616-642.

28. Niven, D. (2012) An Interesting Bias: Lessons from an Academic's Year as a Reporter. Political Science and Politics, 45(2), 259264.

29. Goldenberg, E., Kahn, K. F. (1991) The Media: Obstacle or Ally of Feminists? Annals of the American Academy of Political and Social Science 515, 104-113.

30. Brians, C. L., Wattenberg, M. P. (1996) Campaign Issue Knowledge and Salience: Comparing Reception from TV Commercials, TV News and Newspapers. American Journal of Political Science 40(1), 172-193.

31. D'Alessio, D., Allen, M. (2000) Media bias in presidential elections: a meta-analysis. Journal of Communication 50(4), 133-156.

32. Gidengil, E., Everitt, J. (2003) Talking Tough: Gender and Reported Speech in Campaign News Coverage. Political Communication 20, 209-232. 
33. Arnold, D. R. (2004) Effects of Newspaper Coverage on Citizens, in Congress, the Press, and Political Accountability, 221-243, Princeton University Press, Princeton.

34. Holman, M. R., Merolla, J. L., Zechmeister, E. J. (2016) Terrorist Threat, Male Stereotypes, and Candidate Evaluations. Political Research Quarterly 69(1), 134-147.

35. Dittmar, K. (2015) Navigating Gendered Terrain: Stereotypes and Strategy in Political Campaigns. Temple University Press, Philadelphia.

36. Cassino, D. (2016) Gender Is Costing Hillary Clinton Big among Men. US APP. bttp://eprints.lse.ac.uk/65949/1/blogs.lse.ac.uk (accessed November 19, 2016).

37. McDermott, M. L. (1998) Race and Gender Cues in Low-Information Elections. Political Research Quarterly 51(4), 895-918.

38. Prior, M. (2005) News vs. Entertainment: How Increasing Media Choice Widens Gaps in Political Knowledge and Turnout. American Journal of Political Science 49, 577-592.

\section{ABOUT THE STUDENT AUTHOR}

Karen Bjerre graduated from Sewanee: The University of the South in 2017, with a Bachelor of Arts in politics. This project was presented as her honors thesis. She currently attends Copenhagen University, Denmark, where she is working towards her MSc in political science.

\section{PRESS SUMMARY}

I explore print media coverage of 20 different gubernatorial races between 2004 and 2014 to discover how the presence of a woman candidate in a political campaign affects its media coverage. I find that races with a woman candidate have fewer news stories that cover policy issues than races with two men candidates. I also find differences in how men and women candidates are described in the media: women candidates are more likely to be described as able to "change government," while men candidates are more likely to be described as "experienced." Previous research suggests that voters favor traditionally masculine traits over traditionally feminine traits when evaluating candidates for public office, which means that the difference in media coverage may therefore influence women candidates' chances of getting elected, and thereby women's role in government.

\begin{tabular}{|c|c|c|c|c|c|c|}
\hline \multicolumn{7}{|l|}{$\begin{array}{l}\text { APPENDIX } \\
\text { Table } 1\end{array}$} \\
\hline Contest Pair & Name & Party & Gender & Year & State & $\%$ Vote \\
\hline AK/MT-0 & Sarah Palin & Republican & $\mathrm{F}$ & 2006 & $\mathrm{AK}$ & 48.33 \\
\hline AK/MT-0 & Tony Knowles & Democrat & M & 2006 & $\mathrm{AK}$ & 40.97 \\
\hline AK/MT-0 & Brian Schweitzer & Democrat & $\mathrm{M}$ & 2004 & MT & 50.44 \\
\hline AK/MT-0 & Bob Brown & Republican & $\mathrm{M}$ & 2004 & MT & 46.02 \\
\hline FL/VA-1 & Rick Scott & Republican & $\mathrm{M}$ & 2010 & FL & 48.9 \\
\hline FL/VA-1 & Alex Sink & Democrat & $\mathrm{F}$ & 2010 & FL & 47.7 \\
\hline FL/VA-1 & Terry McAuliffe & Democrat & $\mathrm{M}$ & 2013 & $\mathrm{VA}$ & 47.75 \\
\hline FL/VA-1 & Ken Cuccinelli & Republican & $\mathrm{M}$ & 2013 & $\mathrm{VA}$ & 45.23 \\
\hline MA/VT-1 & Peter Shumlin & Democrat & $\mathrm{M}$ & 2010 & $\mathrm{VT}$ & 49.5 \\
\hline MA/VT-1 & Brian Dubie & Republican & $\mathrm{M}$ & 2010 & $\mathrm{VT}$ & 47.7 \\
\hline $\mathrm{MA} / \mathrm{VT}-1$ & Charlie Baker & Republican & $\mathrm{M}$ & 2014 & MA & 48.4 \\
\hline MA/VT-1 & Martha Coakley & Democrat & $\mathrm{F}$ & 2014 & MA & 46.5 \\
\hline $\mathrm{MO} / \mathrm{ID}-0$ & Butch Otter & Republican & $\mathrm{M}$ & 2006 & ID & 52.67 \\
\hline $\mathrm{MO} / \mathrm{ID}-0$ & Jerry Brady & Democrat & $\mathrm{M}$ & 2006 & ID & 44.11 \\
\hline $\mathrm{MO} / \mathrm{ID}-0$ & Matt Blunt & Republican & $\mathrm{M}$ & 2004 & $\mathrm{MO}$ & 50.83 \\
\hline MO/ID-0 & Claire McCaskill & Democrat & $\mathrm{F}$ & 2004 & $\mathrm{MO}$ & 47.85 \\
\hline $\mathrm{NC} / \mathrm{FL}-0$ & Charlie Crist & Republican & $\mathrm{M}$ & 2006 & FL & 52.2 \\
\hline NC/FL-0 & Jim Davis & Democrat & $\mathrm{M}$ & 2006 & FL & 45.1 \\
\hline NC/FL-0 & Beverly Perdue & Democrat & $\mathrm{F}$ & 2008 & $\mathrm{NC}$ & 50.27 \\
\hline NC/FL-0 & Pat McCrory & Republican & $\mathrm{M}$ & 2008 & $\mathrm{NC}$ & 46.88 \\
\hline NM/OR-1 & Susana Martinez & Republican & $\mathrm{F}$ & 2010 & NM & 53.29 \\
\hline
\end{tabular}




\begin{tabular}{|c|c|c|c|c|c|c|}
\hline NM/OR-1 & Diane Denish & Democrat & $\mathrm{F}$ & 2010 & NM & 46.55 \\
\hline NM/OR-1 & John Kitzhaber & Democrat & M & 2010 & OR & 49.29 \\
\hline NM/OR-1 & Chris Dudley & Republican & M & 2010 & OR & 47.77 \\
\hline NV/IA-0 & Chet Culver & Democrat & M & 2006 & IA & 54.02 \\
\hline NV/IA-0 & Jim Nussle & Republican & M & 2006 & IA & 44.38 \\
\hline NV/IA-0 & Jim Gibbons & Republican & M & 2006 & $\mathrm{NV}$ & 47.93 \\
\hline $\mathrm{NV} / \mathrm{IA}-0$ & Dina Titus & Democrat & $\mathrm{F}$ & 2006 & NV & 43.92 \\
\hline RI/MD-1 & Larry Hogan & Republican & M & 2014 & $\mathrm{MD}$ & 51 \\
\hline RI/MD-1 & Anthony Brown & Democrat & M & 2014 & $\mathrm{MD}$ & 47.2 \\
\hline RI/MD-1 & Gina Raimondo & Democrat & $\mathrm{F}$ & 2014 & RI & 40.7 \\
\hline RI/MD-1 & Allan Fung & Republican & M & 2014 & RI & 36.2 \\
\hline SC/PA-1 & Tom Corbett & Republican & M & 2010 & $\mathrm{PA}$ & 54.49 \\
\hline SC/PA-1 & Dan Onorato & Democrat & M & 2010 & PA & 45.51 \\
\hline SC/PA-1 & Nikki Haley & Republican & $\mathrm{F}$ & 2010 & SC & 51.4 \\
\hline SC/PA-1 & Vincent Sheheen & Democrat & M & 2010 & SC & 47 \\
\hline WA/VA-0 & Tim Kaine & Democrat & M & 2005 & VA & 51.72 \\
\hline WA/VA-0 & Jerry Kilgore & Republican & M & 2005 & VA & 45.99 \\
\hline WA/VA-0 & Christine Gregoire & Democrat & $\mathrm{F}$ & 2004 & WA & 48.873 \\
\hline WA/VA-0 & Dino Rossi & Republican & M & 2004 & WA & 48.868 \\
\hline
\end{tabular}

Overview of coded variables

Basic Data: Days before election; Tone (positive, neutral, negative); Mentions candidate's gender; Horse race coverage; Issue story

Candidate's Appearance: Positive mention; Neutral mention; Negative mention

Policy Mentions (F): Abortion; Child care; Education; Health care

Policy Mentions (M): Crime; Deficit; Economy; Immigration; National security

Trait Mentions (F): Change government; Compassionate; Consensus building; Honest

Trait Mentions (M): Decisive; Experienced; Intelligent; Leadership

Voter Social Group: Gender; Other

\section{Inter-coder reliability}

Two undergraduate students each spent 10 hours to code 10 percent of the primary dataset in order to measure intercoder reliability. Krippendorff's alpha for each individual variable is noted, with the total number of mentions in the primary dataset noted in parentheses (out of 1152).

\begin{tabular}{|c|c|c|c|c|}
\hline $\begin{array}{l}\text { Candidate's gender } \\
0.662 \\
(44)\end{array}$ & $\begin{array}{l}\text { Education } \\
0.798 \\
(261)\end{array}$ & $\begin{array}{l}\text { Immigration } \\
0.891 \\
(83)\end{array}$ & $\begin{array}{l}\text { Honest } \\
0.487 \\
(30)\end{array}$ & $\begin{array}{l}\text { Social group: Gender } \\
0.602 \\
(34)\end{array}$ \\
\hline $\begin{array}{l}\text { Horse race story } \\
0.576 \\
(808)\end{array}$ & $\begin{array}{l}\text { Health care } \\
0.751 \\
(145)\end{array}$ & $\begin{array}{l}\text { National security } \\
0.628 \\
(46)\end{array}$ & $\begin{array}{l}\text { Decisive } \\
0 \\
(0)\end{array}$ & $\begin{array}{l}\text { Social group: } \\
\text { Other } \\
0.792 \\
(72)\end{array}$ \\
\hline $\begin{array}{l}\text { Issue story } \\
0.576 \\
(344)\end{array}$ & $\begin{array}{l}\text { Crime } \\
0.877 \\
(59)\end{array}$ & $\begin{array}{l}\text { Change government } \\
0.259 \\
(19)\end{array}$ & $\begin{array}{l}\text { Experienced } \\
0.168 \\
(52)\end{array}$ & \\
\hline $\begin{array}{l}\text { Abortion } \\
0.835 \\
(96)\end{array}$ & $\begin{array}{l}\text { Deficit } \\
0.446 \\
(39)\end{array}$ & $\begin{array}{l}\text { Compassionate } \\
1 \\
\text { (1) }\end{array}$ & $\begin{array}{l}\text { Intelligent } \\
1 \\
\text { (9) }\end{array}$ & \\
\hline Child care & Economy & Consensus building & Leadership & \\
\hline
\end{tabular}



0.190
0.727
0
0.557

As the table shows, several of the variables have very few mentions in the primary dataset, which helps explain the extreme alpha values. Additionally, some of the latent variables, especially those involving trait descriptions are more difficult to code consistently than specific policy mentions.

\section{CODEBOOK}

Basic data

Mentions candidate's gender - Does the article mention that the candidate is a woman (or man)? If so, it's a 1. if not, please put a 0. Examples of this would be "first female candidate" and similar statements. For this variable, it is important to note that there are both men and women candidates in the dataset (the 'gender' column will tell you which). Only code this variable a 1 if the gender reference is about that specific candidate. For example, an article that is a part of the Tony Knowles dataset would not be coded 1 is it mentions that Sarah Palin is a woman.

Horse race coverage - Every article will be classified as either horse race coverage or issue story. Horse race stories include articles detailing campaign events, stories about polls, debate times, and similar stories.

Issue story - This is the opposite of a horse race story. These will generally detail specific policy positions/issues facing the state. Remember that one of these (horse race) columns will be a 1 and the other a 0 for every article.

Policies

Note that articles do not have to be *about* the issue to be coded as mentioning them.

Abortion - Does the article mention abortion? If so, it is a 1. If not, it is a 0 . Mentions of being "pro-life/choice" also qualify.

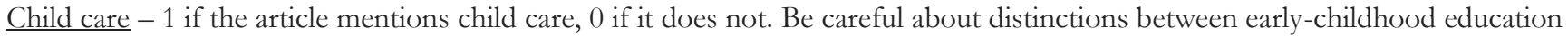
(not child care) and child care.

Education -1 if the article mentions education, 0 if it does not. Be aware that mentions of 'education' in a person's title, for example "board of education," "education supervisor," etc., do not qualify as mentions of education.

Health care -1 if the article mentions health care, 0 if it does not.

Crime -1 if the article mentions crime, 0 if it does not.

Deficit -1 if the article mentions the deficit, 0 if it does not. This variable is about the budget deficit, so be aware that for example deficits in polls do not qualify.

Economy -1 if the article mentions the economy, 0 if it does not. Variations such as "the economic downturn" and the like also count.

$\underline{\text { Immigration }}-1$ if the article mentions immigration, 0 if it does not.

National security -1 if the article mentions national security, 0 if it does not. These will typically prop up as discussions about the Iraq war/terror threat.

Traits

Note that the trait mentions do not have to reference the specific candidate. References to their opponents/other politicians also count. Also, the negative versions of adjective (dishonest, inexperienced, etc) also count.

Change government - If the article mentions someone “changing the way the government works," it's a 1. If not, it's a 0 . This one can have some more variations, but use your best judgment.

Compassion - If the article mentions someone being compassionate, it is a 1 . If not, it is a 0 .

Consensus building - If the article mentions someone being consensus building, it is a 1 . If not, it is a 0 .

Honest - If the article mentions someone being honest, it is a 1 . If not, it is a 0 .

Decisive - If the article mentions someone being decisive, it is a 1 . If not, it is a 0 .

Experienced - If the article mentions someone being experienced, it is a 1 . If not, it is a 0 .

Intelligent - If the article mentions someone being intelligent, it is a 1 . If not, it is a 0 .

Leadership - If the article mentions someone's leadership qualities, it is a 1 . If not, it is a 0 . There might be some variation along the lines of "strong leader," and such, which counts. On the other hand, mentions of the congressional leadership and the like do not count.

Voter social group

Gender - If the article mentions something about women voters (“the woman vote," "doing well with women"), it's a 1. If not, it's a 0 .

Other - If the article mentions specific demographic voter groups other than gender (race, religion, etc.), it's a 1 . If not, it's a 0. 
Print Edition ISSN 1536-4585

Online Edition ISSN 2375-8732 